NBSIR 82-2452(R)

Center for Materials Science

\title{
Polymer \\ Science and \\ Standards \\ Division
}

\section{Annual Report 1981}

00
100
.056
$82-2452(2)$

1981
U.S. DEPARTMENT

OF COMMERCE

National Bureau

of Standards 

POLYMER SCIENCE AND STANDARDS DIVISION

Annual Report 1981

U. S. Department of Commerce National Bureau of Standards Polymers B 1dg. (224), A305 Washington, DC 20234 (301) 921-3734

March 1982 


M. G. BROADHURST - TASK LEADER

- Polymer Tape Insulation for $0 i 1$ Impregnated AC Power Transmission Cables; F. A. Khoury, F. I. Mopsik, S. J. Kryder, and L. H. Bolz . . . . . . . . .

- Polymer Insulation for Superconducting AC Power Transmission Cables; F. A. Khoury, F. I. Mopsik, S. J. Kryder, and L. H. Bolz . . . . . . . . . .

- NBS Workshop on Morphological Studies of Power Cable Insulation Failure; M. G. Broadhurst, F. A. Khoury, A. J. Bur, and G. T. Davis . . . . . . . . . 5

- Space Charge Studies; A. S. DeReggi and F. I. Mopsik . . . . . . . . . . .

- Structure and Performance of Piezoelectric Polymers; G. T. Davis and M. G. Broadhurst ............................

- Aging of Piezoelectric Polymers and Application to Biomedical Uses; D. E. DeRossi, S. C. Roth, A. S. DeReggi, and M. G. Broadhurst . . . . . . .

- Automated Dielectric Measurements; F. I. Mopsik, S. J. Kryder, and S. C. Roth . . . . . . . . . . . . . . . . . . . . . . . .

- Depolarization of Poled PVDF Specimens With Thick Electrodes; A. J. Bur and A.K. Tsao . . . . . . . . . . . . . . . . . . . . . . . .

- Fabrication of Thick Piezoelectric Polymer Film; A. J. Bur, M. G. Broadhurst, and A. K. Tsao . . . . . . . . . . . . . . . . . . . . .

- Acoustic Sensors; A. S. DeReggi, S. C. Roth, and S. Edelman ..........

- Polymer Stress Gauge for Intaglio Printing Press; M. G. Broadhurst, S. C. Roth, S. J. Kryder, A. S. DeReggi, and S. Edelman . . . . . . . . .

- Resistivity and Piezoelectric Measurement on Oriented and Unoriented PVDF Films; A. J. Bur............................

- Nondestructive Evaluation; S. Edelman and S. C. Roth . . . . . . . . . 10

- ASTM Activities; W. P. Harris . . . . . . . . . . . . . . . 10

- The Crystal Structure and Disorders of the Low Temperature Phase (II) of Polytetrafluoroethylene; J. J. Weeks, E. S. Clark, R. K. Eby, and

B. L. Farmer . . . . . . . . . . . . . . . . . . . .

III. MIGRATION AND THE DURABILITY OF POLYMERS

L. E. SMITH - TASK LEADER

- Hydrolytic Degradation of Polyester Polyurethane Elastomers; D. W. Brown, R. E. Lowry, and L. E. Smith . . . . . . . . . . . . . . . . .

- Long Term Aging Studies With Poly(Ethylene Terephthalate); D. W. Brown, R. E. Lowry, and L. E. Smith . . . . . . . . . . . . . . . . . . .

- Oxidation of Polystyrene; B. Dickens . . . . . . . . . . . . . . . 13

- Variable Heating Rate Thermogravimetry; J. H. Flynn . . . . . . . . . . 13

- Analysis of the Kinetics of Thermogravimetry; J. H. Flynn . . . . . . . . 15

- Thermogravimetric Studies of Polymer Degradation; B. Dickens . . . . . . . 16 
- Measurement of Antioxidant Diffusion by Microfluorimetry; $S$. L. Wunder, F. W. Wang, and R. A. Velapoldi ...................

- Migration of 01 igomers and Antioxidants From Polyolefins; S. S. Chang, W. J. Pummer, J. R. Maurey, and L. E. Smith . . . . . . . . . . . . . .

- Measurements of Diffusion in Polymers by Inverse Gas Chromatography; G. A. Senich . . . . . . . . . . . . . . . . . . . . .

- Measurement of Translational Diffusion of Polymers by Fluorescence Photobleaching Recovery Technique; F. W. Wang and W. H. Grant . . . . . . . .

- Assessment of Transport Mechanisms for Radon in and Through Building Materials; R. J. Rubin ....................

IV. MECHANICAL DURABILITY OF POLYMERS FOR DESIGN AND USE . . . . . . . . . . . .

\section{B. M. FANCONI - TASK LEADER}

- Molecular Processes Associated With Mechanical Deformation and Failure; B. M. Fanconi, D. L. VanderHart, S. L. Wunder, J. P. Colson, S. J. Kryder, E. E. Toth, K. L. DeVries, S. Abbate, and G. Zerbi . . . . . . . . . . .

- Relationship Between Morphology and Mechanical Properties of Polyethylene; G. B. McKenna, F. A. Khoury, J. M. Crissman, L. H. Bolz, G. W. Bullman, and G. M. Martin ..........................

- Fracture Processes in Polymers; E. Passaglia . . . . . . . . . . . . .

- Instability and the Phenomena of Necking; L. J. Zapas, J. M. Crissman, $B$. Bernstein, and B. D. Coleman ....................

- Test Method Development; J. M. Crissman, D. L. Hunston, E. A. Kearsiey, R. W. Penn, L. J. Zapas, G. M. Martin, and J. L. Rushford . . . . . . . .

- Non-Linear Behavior of Polymeric Materials; L. J. Zapas, G. B. McKenna, and B. D. Coleman ......................

- Durability of Paper; J. C. Smith . . . . . . . . . . . . . . . .

- Mechanical and Transport Properties of Polyethylene in Organic Solvents;

J. C. Phizlips and A. Peterlin .................

V. POLYMER STANDARDS FOR CONTROL AND EQUITY . . . . . . . . . . . . . . . . .

\section{C. SANCHEZ - TASK LEADER}

- Standards for Gas Transmission Measurements; J. D. Barnes . . . . . . . .

- ASTM Activities; J. D. Barmes . . . . . . . . . . . . . . . .

- Self Calibrating Gel Permeation Chromatogrpahy; P. H. Verdier. . . . . . .

- Dynamics of Polymer Chains; P. H. Verdier and D. E. Kranbueh I . . . . . .

- Low Shear Rate-High Temperature Rotational Viscometer; H. L. Wagner and J. G. Dizzon ...........................

- Rubber and Rubber Compounding Standard Reference Materials; G. W. BuIZman and G. B. MeKenna ..........................

- Static and Dynamic Configurational Properties of Polymer Chains: Theory; F. L. McCrackin, R. J. Rubin, C. C. Han, J. Mazur, I. C. Sanchez, and

D. J. Lohse . . . . . . . . . . . . . . . . . . . . . .

- Polymer Blends; I. C. Sanchez, F. W. Wang, and R. E. Lowry . . . . . . . .

- Static and Dynamic Scattering From Polymers; C. C. Han, D. B. Minor, H. L. Wagner, A. Z. Akcasu, S. J. Bai, D. J. Lohse, and T. P. Lodge. . . . . 
VI. TESTS, STANDARDS, AND CHARACTERIZATION FOR MANUFACTURING AND DURABILITY OF DENTAL AND MEDICAL MATERIALS

\section{J. M. CASSEL - TASK LEADER}

- Dental Composite Chemistry and Development; G. M. Brauer, J. M. Antonucci, J. W. Stansbury, J. M. Cassel, R. L. Bowen, H. Argentar, D. N. Misra,

T. Chen, and E. E. Toth . . . . . . . . . . . . . . . . . .

- Development of Adhesive Bonding Technique and Dental Cements; G. M. Brauer, R. L. Bowen, J. M. Antonucci, D. N. Misra, J. W. Stansbury, E. Cobb, and

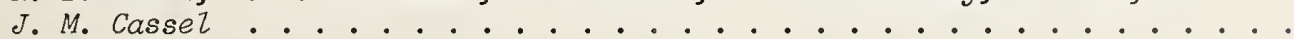

- Wear Resistance and Mechanical Properties of Dental Materials; W. Wu, J. E. McKinney, J. M. Cassel, E. E. Toth, and E. Cobb..............

- Porcelain Fused to Base Metal Alloys and Dental Ceramics; J. A. Tesk, R. W. Hinman, W. de Rijk, E. E. Parry, D. L. Menis, and G. E. O. Widera. . .

- Dental Casting Alloys; J. A. Tesk, R. M. Waterstrat, R. W. Hinman, and E. E. Parry . . . . . . . . . . . . . . . . . . . . . . .

- Clinical and Laboratory Investigation of Dental Amalgams; N. W. Rupp, G. C. Paffenbarger, and $R$. M. Waterstrat . . . . . . . . . . . . . .

- Dental Chemistry and Preventive Dental Treatments; W. E. Brown, L. C. Chow, M. Mathew, M. S. Tung, G. L. Vogel, S. Takagi, and T. M. Gregory . . . . .

- Development of Improved Field Dental Composite Restorative Materials for Military Personnel; G. M. Brauer, N. W. Rupp, J. W. Stansbury, K. Dermann, and J.M. Cassel . . . . . . . . . . . . . . . . . . . .

- Adhesion and Surface Coverage of Synthetic Polymers on Solid Substrates; R. E. Dehl and W. H. Grant ........................

- Characterization of Porosity of Porous Implant Materials; $R$. E. Dehl, W. H. Grant, and J.M. Cassel.....................

- Dental and Medical Materials Standards; J. M. Cassel, J. A. Tesk, G. M. Brauer, and N. W. Rupp .......................

VII. OTHER DIVISION ACTIVITIES

- Invited Talks . . . . . . . . . . . . . . . . . . . 55

- Seminars for Staff and Guests . . . . . . . . . . . . . . . . 63

- Technical and Professional Committee Participation and Leadership . . . . . 68

- Publications . . . . . . . . . . . . . . . . . . . . . . 73

- Patents . . . . . . . . . . . . . . . . . . . . 81

- Workshop .. . . . . . . . . . . . . . . . . . . . . 81

- External Recognition ............................ 82 

Ronald K. Eby, Chief

Leslie E. Smith, Deputy Chief

S. M. Thomas, Administrative Officer

In the seven decades since Leo $H$. Baekeland introduced the first commercial synthetic plastic produced entirely by the reaction of small molecules, polymers have left little of our economy, technology, industry, and culture untouched. We have moved rapidly into an age in which an evergrowing number of humanity's needs-are served by polymers. The volume currently produced exceeds that of steel and forms the basis of industries which add over 90 billion dollars of value by manufacture (a measure of the relative economic importance of manufacturing among industries) and provide 3.4 million jobs. Recent summaries show that polymers research already accounts for about 47 percent of the total industrial R\&D expenditure for metals, polymers, and inorganic materials. Among these materials, polymers also constitute about 39 percent of the value added by manufacture, 49 percent of the jobs, 45 percent of the number of scientific publications, and 39 percent of the ASTM standards.

Polymers have been shaped by intellectually sophisticated research which has been recognized by a number of Nobel Prizes. The resulting materials have contributed to national industrial productivity through their economy, ease of fabrication, and useful properties such as low density, corrosion resistance, and toughness. Polymers are energy efficient; the largest volume polymer, polyethylene, uses 28 percent of the energy per unit volume required by steel for conversion from raw to refined material and yields further savings in processing and shipping. In fact, studies have shown that for every barrel of oil used to make plastic products, approximately one barrel is saved in comparison with the use of the next best alternative material. These useful properties have enabled polymers to contribute to increased productivity both by creating entirely new products and by substituting for other materials in existing products.

Polymers have entered increasingly sophisticated and demanding applications. Polyvinylidene fluoride films are used as electromagnetic transducers in high fidelity systems. Structural parts of aircraft are constructed from composites with a polymer matrix. Polyurethane elastomers are used to pump blood in circulatory assist devices. The list of such examples is very long, and inciudes trends for the increased use of polymers in packaging, construction, transportation, national security, electronics, information handling, clothing, appliances, energy, dentistry, and medicine. Since polymer technology is on the steep part of the learning curve, these trends create many new needs for concepts, measurements, standards, and data that can be used to ensure their reliable performance in the use, processing, and other phases of the material cycle.

To identify the key needs, the managers of the Polymer Science and Standards Division make structured visits to industrial and Government laboratories. Visited sites include Bell Labs, DuPont, Johnson and Johnson, Army R\&D Command, General Motors, Boeing, and Lawrence Livermore Laboratories. Surveys have been conducted in the areas of durability, composites, and processing. These and other contacts with research associates, experts in industry, trade associations, standards associations, guest workers, Government, and academia ensure that the Division's efforts are directed toward fostering the effective use of polymers in solving national problems. These national problems include the growth of industrial productivity, national security, efficient Government, materials utilization, conservation of critical materials, and reasonable regulation of the effects of technology.

The Division has used these activities as a basis for participating in budget initiatives to secure the resources necessary to meet emerging needs. For the budget cycles currently active, programmatic initiatives emphasize polymer matrix composites because of their role in increasing industrial productivity and substituting for critical materials. Specific areas encompass toughened hybrid matrix materials, high strength polymer fibers, methods of predicting durability, and methods of measuring and controlling matrix cure. Competence building initiatives on the science of forced Rayleigh scattering, polymer morphology, and time-resolved Fourier transform infrared spectroscopy have been prepared.

Current activities are associated into areas concerned with mechanical reliability in load-bearing applications, chemical durability and lifetime prediction including the migration of protective additives, characterization methods and standards for molecular composition and use properties, dielectric properties and electrical conduction, and reliable dental and medical materials. Substantial cooperative efforts are conducted with research associates from a number of industries, the Department of Defense, the National Institutes of Health, the American Dental Association, the Food and Drug Administration, and the Bureau of Engraving and Printing. All these efforts lead the scientists of the Division into productive examination of those properties of polymers which are of concern to people who use, design, and produce useful polymer objects. This work helps society capture, in safe and satisfying ways, the benefits of the increasingly widespread application of polymers. 
In the following pages, the activities of the Division are presented formally in five Tasks, each described separately:

DIELECTRIC PLASTICS DESIGN AND PERFORMANCE

MIGRATION AND THE DURABILITY OF POLYMERS

MECHANICAL DURABILITY OF POLYMERS FOR DESIGN AND USE

POLYMER STANDARDS FOR CONTROL AND EQUITY

TESTS, STANDARDS, AND CHARACTERIZATION FOR MANUFACTURING AND DURABILITY OF DENTAL AND MEDICAL MATERIALS 


\section{G. Broadhurst}

\section{Task Leader}

Trends in dielectric application of polymers include use of higher working voltages in electrical apparatus and transmission lines to conserve materials, save space, and reduce weight. This work provides manufacturers (polymer manufacturers, film fabricators, transducer manufacturers), trade associations (EPRI), and Government agencies (DOE, DOD) with data, measurement methods, and concepts which identify limitations in polymer performance traceable to manufacturing practices. For example, steam curing of extruded polyethylene insulated cable results in lifetime-limiting voids and inclusions. With the trend toward increased use of underground transmission of electric power, improved insulation performance is required. To meet these needs, NBS develops measurement methods and performs measurements to obtain required data, holds workshops to improve research efforts in the area of power cable and capacitor insulation, recommends test methods for new material selection, and carries out related activities. Data and recommendations from this work go directly to cable manufacturers and funding organizations (i.e., EPRI, DOE) who are able to specify and require manufacturing changes leading to improved products.

Applications of polarized polymers as pyro- and piezoelectric transducers are rapidly growing. Transducer manufacturers as well as private and Government transducer users (DOD, EPA, HHS) need reliable information connecting polymer structure and polymer performance to the design of improved transducers. This work helps transducer material and device manufacturers design and develop commercial polymer transducers for use in health and safety applications (pulse monitors, therapeutic and diagnostic sound measurements, auto crash sensors, prosthetic fitting devices, nontoxic antifouling coatings, mine disaster warning devices), and in the areas of national security (microphones, hydrophones, intrusion detectors, ultrasonic and infrared imaging devices, fusing devices, etc.). Important scientific opportunities of this work include breakthroughs in understanding the basic factors in electrical failure of polymers and discovery of novel measurement techniques which are becoming possible with newly developed polymers. For example, on-going work will show how polymers interact with electronic and ionic charges and how structure can be modified to optimize piezoelectric and semiconducting properties.

The study of electrical conduction of organic polymers is a major new frontier in solid state physics. Most current research involves an empirical search for improved dopant-polymer combinations from a bewildering array of compounds. Chemical structure-electrical property correlations are not yet evident. The key need is for a better knowledge of the molecular packing, dopant locations, chemical changes with doping, molecular orientation, and charge carrier pathways to form a basis for modeling and predicting electrical properties. This work is ideally suited to NBS because it involves improved measurements, standards, and test methods. Polymer conductors have the advantages of being lightweight, flexible, made from abundant raw materials, resource and energy conserving, and inexpensive. Thus, success of the project will have a potentially significant impact on national problems of materials replacement, productivity enhancement, energy conversion and storage (polymer batteries), electromagnetic shielding, and microcircuitry.

Ionic conduction in polymers provides a basis for a large and growing class of important applications including production of chlorine and caustic from brine, hydrogen production from low-head hydroelectric or ocean thermal energy sources, solid electrolytes for fuel cells and batteries, gas sensors, materials separation membranes, and electrochemical devices. NBS work on conducting polymers is aimed at characterizing the polymer structures, measuring their properties, and relating the structures and properties to provide a basis for predicting electrical performance from molecular details.

\section{Polymer Tape Insulation for 0il-Impregnated AC Power Transmission Cables}

\section{F. A. Khoury, F. I. Mopsik, S. J. Kryder, L. H. Bolz}

Brookhaven National Laboratory has established a program to develop an oil-impregnated or oil filled high power ac transmission cable using polymer tape insulation. Until now, oil impregnated paper has been used for large power cables, but the relatively high loss of such cables has generated interest in lower loss systems, such as oil-polymer cables for use at 700 to $1000 \mathrm{kV}$.

A project sponsored by Brookhaven with DOE funds has been established in the Polymer Science and Standards Division to evaluate the suitability of polymer films. The aims of this project are: the measurement of the dielectric constant and dielectric loss of candidate films between $273 \mathrm{~K}$ and $403 \mathrm{~K}$, the study of the fine structural characteristics of the films, and the specification of the most suitable films for the intended end use.

Measurements this past year have been carried out on polyethylene and polypropylene tapes prepared for Brookhaven by Battelle Institute. They were prepared by rolling at temperatures from 293 to $398 \mathrm{~K}$ for polyethylene with thickness reduction ratios of four to ten and at a temperature of $423 \mathrm{~K}$ for 
polypropylene with thickness reduction ratios of five to six. Two different base polymers were used for both the polyethylene series and the polypropylene series. The dielectric measurements were carried out under standard temperature cycling, and density measurements were carried out on the tapes both before and after the dielectric measurements to check on their stability under thermal cycling.

All the polyethylene tapes showed a significant decrease in loss (tan $\delta$ ) on thermal cycling. The decrease at $303 \mathrm{~K}$ (after heating incrementally to $373 \mathrm{~K}$ over a period of 24 hours) was about a factor of two. Aside from this decrease, the losses in one base polymer were substantially lower than in the others (less than $24 \times 10^{-6}$ on the third heating cycle). All the tapes increased in density upon thermal cycling, but the tapes rolled at $398 \mathrm{~K}$ showed a much smaller change, which indicates their higher mechanical stability.

The polypropylene tapes, which were processed at a roll temperature of $423 \mathrm{~K}$ (the maximum temperature of the dielectric measurements) showed behavior dependent on which of two base polymers was used. Tapes made from one base polymer showed little variation with thermal cycling and had a loss maximum at $350 \mathrm{~K}$ of $35 \times 10^{-6}$. Tapes made from the other polymers showed a loss peak which shifted from $320 \mathrm{~K}$ to $370 \mathrm{~K}$ upon thermal cycling and which had a maximum value of about $800 \times 10^{-6}$. Tapes from both polymers showed an increase in density after the thermal cycling, showing that they had not been fully stabilized at the rolling temperature.

Brookhaven uses the NBS data to select the most suitable film, including specially oriented or bonded films, for new synthetic-tape-insulated power cables. The development of such cables has been identified by EPRI as an important short-term research objective in meeting increasing U.S. electric power requirements.

This project was completed during FY81 and a final report was prepared.

Polymer Insulation for Superconducting AC Power Transmission Cables

F. A. Khoury, F. I. Mopsik, S. J. Kryder, and L. H. Bolz

This project is funded through Brookhaven National Laboratory by DOE. The goal has been to evaluate the suitability of polymer films for use as electrical insulation in a superconducting ac power cable that would operate at 6.0 to $8.0 \mathrm{~K}$. Development of a superconducting power cable has been identified by EPRI has a long-range research goal in order to meet future demands for very high power transmission (several giga watts). The measurements provided by NBS have enabled Brookhaven to specify special composite tapes which are now being built into prototype cables.

The primary task in this project has been the measurement of the dielectric constant ( $\varepsilon$ ) and dielectric loss $(\tan \delta)$. For suitable insulation these should be less than 2.5 and $20 \times 10^{-6}$ respectively at 6.0 to $8.0 \mathrm{~K}$ and $60 \mathrm{~Hz}$. These measurements have been coupled with studies of the fine structure of the films (crystallinity, molecular orientation, voids) to guide the development of optimum films. In addition, measurements of the compressibility of stacks of polymer films have been made to provide data necessary for winding tapes sliced from the films onto the cable core.

In FY81, measurements were made on a series of laminates made from polypropylene films of 40 to $60 \mu \mathrm{m}$ thickness bound together by thin polyurethane layers to provide total thicknesses of 80 to $180 \mu \mathrm{m}$. These laminates have shown promise of meeting all necessary requirements in work done in previous years. One series of measurements was made on a set of laminates graded in thickness. Three were bilaminates of total thickness 80,100 , and $120 \mu \mathrm{m}$. All the films had suitably low loss levels with tan $\delta$ ranging from $10-15 \times 10^{-6}$. Low loss was achieved by use of a polypropylene film that had special antioxidants as well as reduced polyurethane content. Previous work had shown that antioxidants can strongly increase the loss of polypropylene at cryogenic temperatures (< $10 \mathrm{~K}$ ). Also, it was shown that if too much polyurethane is used as a binder, the higher loss levels of the polyurethane cause the performance of the laminates to be unsatisfactory.

Another series of measurements was made on a $94 \mu \mathrm{m}$ thick bilaminate and a $140 \mu \mathrm{m}$ thick trilaminate in which a slip agent had been added to the polypropylene in order to obtain better mechanical properties of the finished cable. Losses increased to 16 to $23 \times 10^{-6}$ at 6.0 to $8.0 \mathrm{~K}$ as a result of this change.

In addition to the dielectric loss measurements, compressibility measurements were made on stacks of the first set of bilaminates, carbon loaded polytetrafluoroethylene, and carbon loaded Kraft paper. These data are needed to ensure proper bendability of a finished power cable.

This project has been completed and funding for further research on the insulation for the superconducting cable terminated. A prototype cable is being built. 
M. G. Broadhurst, F. A. Khoury, A. J. Bur, and G. T. Davis

A two-day workshop on the morphology of polyethylene and its relation to the current state of research on the causes of premature failure of polyethylene insulation on high power transmission cables was held at NBS. The workshop was organized by Martin Broadhurst of the Polymer Science and Standards Division and was jointly sponsored by NBS and the Electric Power Research Institute, the research arm of the U.S. electric utilities. Participants were from electric utilities, cable makers, polymer manufacturers, and universities. In the welcoming address, J. D. Hoffman, Director of NML, described his research and its relevance to both the electrical properties and morphology of polymers. NBS scientist Freddy Khoury delivered the keynote address.

The electric utilities requested this workshop because of their continuing concerns for reducing costs and improving efficiency of electrical transmission systems. A further concern is to minimize space required for power transmission and the environmental impact of transmission lines; both of these considerations favor underground cables, for which polymer-insulated cables operating at higher voltages are attractive solutions provided that improved dielectric performance and durability can be obtained.

Several scientific approaches to eliminating the problem of electric failure in polymers are currently being followed. In one promising approach, morphologists use electron and optical microscopes together with other techniques to examine structural details of the polyethylene cable insulation to deduce reasons for failure and to identify ways to modify processing conditions to achieve better performance. Recent studies have been at odds as to how failure channels which develop during cable operation are related to the chemicals and steam used to cross-link or cure the insulation. There was a consensus that the various etching techniques now being used in cable insulation studies often produce artifact structures on the surface of polyethylene samples which are not representative of the original samples. Many structural interpretations are, therefore, in doubt. Improved methods of carrying out these important measurements to give more reliable results were delineated.

A summary report of the workshop has been prepared by NBS and has been issued as an EPRI publication. This report will be used immediately by EPRI as a guide to planning and funding future research projects on morphology of polyethylene insulated cables. In the longer term, this work will lead to improved processing conditions and better performance from underground electric power cables.

\section{Space Charge Studies}

\section{A. S. DeReggi, F. I. Mopsik}

The measurement of charge distributions in solid polymers is basic to understanding polymer-charge interactions. These interactions are believed to play a key role in a material's insulating or conducting properties, electrical strength, and electrical aging.

During the past year, a thermal pulse method recently developed at NBS has become increasingly accepted as one of the most powerful, readily-available methods for measuring the distribution of charge or polarization in a dielectric film as a function of depth, where the thickness of the film is of the order of $25 \mu \mathrm{m}(0.001 \mathrm{in})$. Further development of the technique at NBS has been primarily in the area of data analyses. A recently acquired transient recorder allows digital acquisition of data which is then transferred to a tape cassette for input to a laboratory minicomputer. A program has been developed and tested to show that as many as ten Fourier coefficients of the charge or polarization distribution can be extracted from the data. This means tht the resolution of the charge or polarization distribution is on the order of one-tenth of the film thickness. These developments which enable us to input data from a tape and output a graph of the polarization distribution have been presented in the Proceedings of the most recent Conference on Electrical Insulation and Dielectric Properties in Boston (1980), and a more extensive article is in review.

The above progress in data analysis has opened the possibility of obtaining for the first time quantitative and unambiguous information about polymer charging phenomena. For instance, we have shown that the piezoelectric polymer, polyvinylidene fluoride (PVDF), when poled at ambient temperature, poles more or less symmetrically but not uniformly. The regions near the surfaces seem to have a greater reluctance to responding to applied poling fields than the bulk of the sample. This is manifested by the form of the polarization distribution obtained as progressively stronger fields are applied in the thickness direction and the changes which occur upon applying progressively stronger fields in the reverse direction. At low forward fields, the polarization in a virgin sample first forms primarily in the bulk. At medium and high fields, it develops near the surfaces in addition to in the bulk. Up to the highest attainable fields, it remains somewhat less ( $\sim 15$ percent) near the surfaces than in the bulk. When the applied field is subsequently reversed, the polarization reverses in the bulk more readily than it does on the surface. A sample which is forward poled at $2.0 \mathrm{MV} / \mathrm{cm}$ and subsequently is reversed poled at $-1.0 \mathrm{MV} / \mathrm{cm}$ exhibits strong forward polarization near the surfaces and strong reverse polarization in the bulk, with practically zero mean polarization. 
These effects are believed to be due to the mechanical constraints imposed during poling by the deposited aluminum electrodes. Investigations now underway will provide additional information concerning the origin of the above effects. These investigations include studies of the dependence on thickness of the Al, thickness of the polymer, and on the type and thickness of the electrode material.

The effects on charging phenomena associated with work function differences between the polymer and metal electrode are also of basic interest and could be important in determining poling behavior. Previous qualitative thermal pulse work at NBS on non-polar polymers strongly indicated differences in charging properties when $A 1$ and $A g$ were used as electrode materials. During a renewed visit to NBS, contractor Prof. Sidney Lang of the Ben Gurion University has been studying the effects of electrode material in the polarization distribution in PVDF poled over a range of applied fields.

We also have begun to apply the thermal pulse method to investigate charging of polymers bombarded by electron beams. These studies have been motivated by interest at Rome Air Development Center on the electron-beam charging of polyvinyl chloride films. Simple models for the radiation effects on the conductivity which are currently being developed at RADC predict nonuniform distributions. To guide this theoretical work, there is a need for experimental determinations of the charge distribution. Our thermal pulse measurements, so far, for $700 \mathrm{kV}$ electrons and a 1 imited dose range (200 to 2000 seconds at $10^{-9} \mathrm{~A} / \mathrm{cm}^{2}$ ) indicate more or less uniform charge distributions at long times after irradiation.

\section{Structure and Performance of Piezoelectric Polymers}

\section{G. T. Davis, M. G. Broadhurst}

The objectives of this task are to study the molecular motions, structural changes, and electrical properties associated with the process whereby polymers are made piezoelectric. Information gained from these studies will be used to optimize piezoelectric and pyroelectric response with respect to both initial activity and long-term stability.

While piezoelectric and pyroelectric activity from polymers is comparable to that from good ceramic materials, other properties of polymers often make them the preferred transducer material. Some of the polymer properties which make them so useful include toughness, flexibility, low density, broad band response, close acoustic impedance match to water, and ease of fabrication. Requests for information from other Government agencies and from industries have remained numerous. Interest in this area of research continues at a high level, as evidenced by the 22 papers on this topic presented at the American Physical Society meeting in March 1981.

A copolymer of vinylidene fluoride and trifluoroethylene ( 52.8 mol \% VDF) has been studied in terms of structural changes that occur upon poling and subsequent depoling by heating through the reported ferroelectric Curie temperature of $70^{\circ} \mathrm{C}$. As crystallized from the melt, the polymer is a mixture of two phases. In one phase, the molecular conformation is thought to be (TG) 3 , resulting in a $3 / 1$ helix as in the trifluoroethylene homopolymer. In the other phase, the molecular conformation is all trans, in which the molecules are packed hexagonally. The electric fields employed in poling $(0.5$ to $1.0 \mathrm{MV} / \mathrm{cm})$ are sufficient to cause a crystal phase conversion to the all trans conformation with considerable improvement in order, as evidenced by a shift to smaller cell dimensions and sharper $x$-ray diffraction maxima. Upon heating, the two coexisting phases initially present undergo large changes in cell dimension between 60 and $70{ }^{\circ} \mathrm{C}$, and only a single $3 / 1$ helical conformation exists above $85^{\circ} \mathrm{C}$. Although the change in conformation from all trans to (TG) 3 which occurs in the vicinity of 60 to $85^{\circ} \mathrm{C}$, can certainly account for the ferroelectric transition; much of the piezoelectricity induced by poling at room temperature is lost before the transition to a single $3 / 1$ helical conformation is observed. It therefore seems probable that polarization is lost by randomization of the dipole direction followed by a change in chain conformation at a somewhat higher temperature.

We have succeeded in varying the small angle x-ray spacing in drawn samples of polyvinylidene fluoride by adjusting the temperature of extension--a processing characteristic reported years ago for polyethylene and polypropylene. The linear position-sensitive detector on the Rigaku Denki rotation anode source was employed to measure long spacings varying from $11.5 \mathrm{~nm}$ to $16.5 \mathrm{~nm}$. These films were prepared to study the effect of lamellar spacing on the poling characteristics of PVDF. 0ver this range of crystal size, there is no effect on the pyroelectric response for poling fields in excess of 2.0 $\mathrm{MV} / \mathrm{cm}$. The ease of switching as determined from hysteresis loops has yet to be measured.

Dielectric and polarization data were compared to calculated results from a ferroelectric theory developed in our laboratory. The model has six equally spaced rotational energy minima. A first-order cooperativity assumption is applied, and the dielectric constant and remnant polarization are calculated as a function of temperature. Comparison between the experimental and calculated results gave good agreement. 


\section{Aging of Piezoelectric Polymers and Application to Biomedical Uses}

\section{E. DeRossi, S. C. Roth, A. S. DeReggi, M. G. Broadhurst}

A new research associate in the Polymer Science and Standards Division has begun work on the aging and durability of piezoelectric polymers for biomedical devices under sponsorship of the Brown University Research Foundation. Dr. Danilo DeRossi, on leave from the University of Pisa where he is on the Faculty of Engineering and a Group Leader in the Institute of Clinical Physiology, sees great potential for such polymers in biomedical uses and has come to NBS to develop measurements of and models for the aging of piezoelectric activity in po?ymers.

The unique properties of piezoelectric polymers--low density, good mechanical impedance match to human tissue, flexibility, nontoxicity, and ease of fabrication in complex patterns and arrays--are ideally suited to biomedical transducer applications. The factors which affect the stability of the piezoelectric activity in polymers are not well understood, however, nor are accelerated tests available to measure the durability of piezoelectricity in these materials. This Research Associate Program will include the following activities: laboratory research to improve techniques for periodic nondestructive measurements of the performance of piezoelectric polymer samples, experiments to aid in the development of an accelerated service test to facilitate evaluation of the relative durability of piezoelectric polymers, design and evaluation of predictive mathematical models relating the molecular and microstructural characteristics of piezoelectric polymers to aging mechanisms, and evaluation and recommended improvements of existing methods for calculating the properties of polycrystalline samples (the commonly available form) from analyses of single crystal results.

Through this program, fundamental polymer transducer science at NBS wil? be transferred to Brown University and other private sector groups which are inventing and developing devices. NBS will gain assistance and impetus from their efforts to develop and improve test methods, data, and concepts regarding durability and aging in the important new class of piezoelectric polymer materials.

This current activity is expected to be followed by other research associate activities related to the use of polymer transducers in areas such as robotics.

\section{Automated Dielectric Measurements}

\section{F. I. Mopsik, S. J. Kryder, and S. C. Roth}

This subtask was begun as part of the power cable insulation subtasks ( 1 and 2 ) to meet the need for more rapid dielectric measurements on large numbers of samples. The instrument being deve?oped will record the transient electric charge response of a dielectric sample to a step change in electric field in real time. This response will automatically be transformed into dielectric constant and loss over the frequency range of $1 \mathrm{mHz}$ to $10 \mathrm{kHz}$ and plotted by computer. The work directly supports Task 12139 by providing a basic tool for measuring electrical properties.

During FY81 the electronic circuits for an automated dielectric spectrometer were completed. A hardware clock programmer was built to allow for compatiblity between the sampling rates needed at the beginning of the transient and the time the computer needs to make changes in the operating conditions during data acquisition. This device provides automatic sequencing through the first of three sampling rates so that the computer has time to take control of the sampling.

The analog-to-digital converter has been built and tested. The converter has successfully operated at speeds compatible with a $5 \mu$ sampling time so that the beginning of the transient can be well characterized and extrapolated to the beginning of the charge transient. Its sensitivity and noise level is such that it will not degrade the performance of the charge detector.

Finally, the first few data acquisition programs have been written, and the interfaces of the components (generator, detector, converter, clock, clock programmer) to the computer have been successfully tested. The initial results show that the desired loss sensitivity (< $10 \mu \mathrm{rad}$ ) can be achieved and that the design goals will be met. The resolution was sufficient to show that one of a pair of $10 \mathrm{pF}$ standard capacitors was faulty and had an apparent negative loss that was confirmed by transformer bridge to be $-70 \mu \mathrm{rad}$ at $100 \mathrm{~Hz}$. For the size of the integrating capacitor used in the detector, this result was well within the sensitivity limits initially desired.

\section{Depolarization of Poled PVDF Specimens with Thick Electrodes}

\section{A. J. Bur and A. K. Tsao}

The effect of electrode thickness on the activity of thin film PVDF was investigated by observing the pyro- and piezoelectric activity of 26 specimens whose electrode thickness varied from 60 to $310 \mathrm{~nm}$. These samples were obtained from Bell Laboratories (Allentown, Pennsylvania) where they were poled under uniform conditions after the entire electrode was deposited on the sample. This subtask 
was done partly in cooperation with Bell Laboratories who are interested in the effects of electrode thickness for telephone switch pad devices and contributes to the NBS task of optimizing transducer material for applications.

The electrodes consisted of two layered metals: first, Ti was deposited to a thickness of $60 \mathrm{~nm}$; Au was then deposited on top of the Ti to a thickness which varied from 15 to $250 \mathrm{~nm}$.

The samples were examined in three experiments: (1) the hydrostatic piezoelectric coefficient and the pyroelectric coefficient were measured for the original samples; (2) both coefficients were measured after the thick electrodes were removed; and (3) the depolarization charge was measured by detecting the discharge as the samples were subjected to an abrupt temperature rise through the melting point. For the second experiment, all samples had $\mathrm{Ti}$ electrodes of $60 \mathrm{~nm}$ thickness. The results of these measurements are: (1) the values of the coefficients for the as-received samples were lower the thicker the electrode; (2) with the thick electrodes removed from the samples, the values were the same as those for the thick electrodes in place; and (3) the depolarization charge decreased as the original electrode thickness increased.

We have concluded that the decrease in piezelectric and pyroelectric response as a function of electrode thickness is essentially due to the decreases in polarization of the thick electroded specimens and that thick electrodes mechanically constrain the sample during poling so that optimum poling cannot be achieved.

\section{Fabrication of Thick Piezoelectric Polymer Film}

A. J. Bur, M. G. Broadhurst, and A. K. Tsao

The subtask results from a direct request from the Navy to explore fabrication of thick piezoelectric polymer film for hydrophone use. Thick film is needed because the voltage response of thin PVDF films is less than typical ceramic hydrophones. The voltage response is inversely proportional to capacitance and hence directly proportional to the thickness of the film. To make the voltage sensitivity of polymers better than that of ceramics the thickness should be at least $750 \mu \mathrm{m}$. To stretch and polarize a film this thick requires special equipment and so far only a couple of European companies have been successful at producing it. In this work, NBS is developing techniques whereby industrial laboratories in the U.S. can produce suitable films.

U1tra-drawn PVDF is material which has been stretched beyond its natural draw ratio of $4: 1$. Using a PVDF sample which had been previously stretched to its natural draw ratio of $4: 1$, it is possible to obtain draw ratios of 6.5 or 7.0 to 1.0 by reclamping the sample in the Instron machine and stretching at $140{ }^{\circ} \mathrm{C}$ at a rate of $0.05 \mathrm{in} / \mathrm{min}$. During the ultra-stretch, the load increases while the sample dimensions decrease, indicating that the modulus increases with increasing draw ratio. The ultra-drawn material is desired because it contains mostly $\beta$ phase material which poles at lower fields and has a larger polarization than other phases, it is highly oriented, and it is able to withstand high poling fields.

Poling these thick samples requires high voltages. For example, to pole a $0.3 \mathrm{~mm}$ thick sample at $2.0 \mathrm{MV} / \mathrm{cm}, 60 \mathrm{kV}$ are needed. Working at these voltages, it is necessary to suppress corona and flashover conduction by carrying out the poling in oil and to construct the sample electrodes so that inigh electric fields are not present at the edges.

\section{Acoustic Sensors}

\section{A. S. DeReggi, S. C. Roth, and S. Edelman}

Probing hydrophones and biomedical sensors made with the piezoelectric polymer polyvinylidene fluoride (PVDF) have been shown to have superior performance characteristics over previously available piezoelectric ceramic devices in terms of a wide operating band-width and negligible insertion effects. These favorable characteristics stem from the similarity in acoustic impedance between the PVDF and the common transmission and test media relevant in ultrasonics, such as water and oils, and in the case of biomedical ultrasonics body tissue. There is currently a growing interest in these polymer transducers in the ultrasonic community in general and in the biomedical community in particular. The collaboration between NBS and a guest worker from the Bureau of Radiological Health (BRH) has continued during the past year. The principal aims of the collaborative work are the development of polymeric acoustical probes for measuring the radiation pattern of biomedical transducers and the development of improved methods of characterizing the performance of existing biomedical transducers using the new polymeric probes. The knowledge obtainable with the new polymeric sensors can be used to ensure both the safety and adequacy of ultrasonic dosage as well as to advance research in ultrasonic treatment.

A variety of ultrasonic probing hydrophones and hydrophone arrays have been constructed, all featuring the spot-poled, tensioned membrane design configuration which was introduced at NBS several years ago and was publicized extensively during the course of the past year through contributions to the Proceedings of the 25th Annual Meeting of the Institute of U1trasound in Medicine (September 1980), 
the 1980 IEEE U1trasonics Symposium (November 1980), the Sixth International Symposium on U1tric Imaging and Tissue Characterization (June 1981), and through the publication of an article $\lfloor 1\rfloor$ addition, BRH has filed a patent application (November 1980), and NTIS has publicized this patent application among ultrasonic manufacturers who might be interested in its commercialization.

The typical active electrode diameter of these probes is around $1.0 \mathrm{~mm}$, although useful output has been obtained from a probe with an active electrode diameter as small as $300 \mu m$. The operating bandwidth of greatest interest is 0.5 to $5.0 \mathrm{MHz}$. Over this range, the frequency response of the polymer sensors is flat, with a sensitivity for a probe with a $1.0 \mathrm{~mm}$ diameter active electrode of $-237 \mathrm{~dB} \pm 1.5 \mathrm{~dB}$ re $1.0 \mathrm{~V} / \mu \mathrm{Pa}$, and an insertion loss not exceeding $2.5 \mathrm{~dB}$. Repeated sensitivity measurements over a period of 2.5 years have indicated good stability with no credible trend for reduced sensitivity within the $\pm 1.0 \mathrm{~dB}$ accuracy of the measurements.

Larger diameter (1.0 cm diameter active area) piezoelectric polymer sensors have been used at NBS for many years to ciemonstrate the capability of piezoelectric polymers to detect the heart pulse when held against the skin at the medically common peripheral monitoring sites. In collaboration with NBS, members of the CNR Institute of Clinical Physiology at the University of Pisa are evaluating piezoelectric sensors for the monitoring of cardiac patients.

Preliminary clinical data on peripheral pulse signals obtained at Pisa with NBS sensors will be presented at the forthcoming Computers in Cardiology Conference in Florence in September 1981. These data appear to give information complementary to but not necessarily contained in EKGs.

Reference:

[1] A. S. DeReggi, S. C. Roth, J. M. Kenney, and S. Ede1man, J. Acoust. Soc. Am. 69, 853 (1981).

Polymer Stress Gauge for Intaglio Printing Press

M. G. Broadhurst, S. C. Roth, S. J. Kryder, A. S. DeReggi, and S. Edelman

This work is supported by the Bureau of Engraving and Printing, U.S. Department of Treasury. The purpose is to develop a gauge to measure the dynamic pressure at critical points in the presses used to print money. The first phase of the work is completed. The accomplishments of the first phase included measurement of the width of the area of contact between the two cylinders of. the press, the duration of contact of a point on the upper cylinder with the lower cyinder, and a qualitative (uncalibrated) determination of the change of the pressure with time during contact.

The second phase of the work is to provide a calibrated pressure transducer and a complete signal processing system for transmitting the transducer signals from the operating printing press and providing pressure and temperature vs time data for the nip (region of contact between the printing rollers). Piezoelectric polymer strips containing a pressure tranducer and thermocouple are placed under the fiber rigging on the top roller of the press. When the polymer strip moves into position between the top and bottom rollers, it is subjected to both a pressure increase and a temperature increase due to adiabatic heating of the trapped and compressed air. The thermocouple signal monitors the temperature change, and the signal processor subtracts from the polymer transducer signal that part which is due to temperature change. The resulting signal is that due to pressure change only. A calibrator has been developed to monitor and correct for changes in transducer response due to aging while the transducer is installed on the press. A final check of the system has been performed between metal plates covered with rigging material and mounted in a hydraulic press. Sudden release of pressure in the hydraulic press simulates the release of pressure in the printing press as the nip moves past the transducer. The pressure measurement system will provide the BEP with data on printing pressures and temperatures which will not only permit better control of printing pressures but also will provide data needed for improving paper and ink.

Successful demonstration of this measurement technique has received the attention of both the paper and synthetic polymer film manufacturers who have a long-standing need to measure and control pressures between the rollers used to make films. Results will be transmitted to these industries.

\section{Resistivity and Piezoelectric Measurement on Oriented and Unoriented PVDF Films}

\section{A. J. Bur}

This subtask directly supports the optimization of piezoelectric polymers for applications in industry and Government. It concerns that part of the transducer preparation where large electric fields are applied to the film in order to polarize it. It has been found that the larger the field, the higher the piezo/pyroelectric activity and this subtask examines the effect of film orientation on the maximum electric field which the material can support.

Thick specimens of PVDF were prepared by molding PVDF powder or pellets in a compression mold under vacuum. These specimens, in an isotropic unoriented condition, were found to be unsuited for 
poling because electrical breakdown occurred at fields between 0.3 and $0.6 \mathrm{MV} / \mathrm{cm}$, which are too $10 \mathrm{w}$ to produce significant polarization. When these samples were mechanically oriented, however, poling fields of $1.0 \mathrm{MV} / \mathrm{cm}$ or higher could be attained so that acceptable transducer activity could be achieved. This much orientation was carried out by means of tensile extension at $55^{\circ} \mathrm{C}$ which produced a natural draw ratio of $4: 1$.

In order to examine the difference between the electrical properties of mechanically oriented and unoriented PVDF, we measured resistivity before and after orientation. The resistivity for an oriented sample at room temperature showed a marked rise by an order of magnitude or more above that of the unoriented samples. For unoriented samples, resistivities were in the range 1.7 to $2.4 \times 10^{15} \mathrm{ohm}-\mathrm{cm}$, whereas for the oriented samples resistivities ranged from $4.2 \times 10^{16}$ to $>2.2 \times 10^{17} \mathrm{ohm}-\mathrm{cm}$.

While we did not establish a correlation between resistivity and electrical breakdown voltage, we

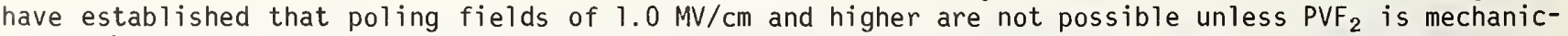

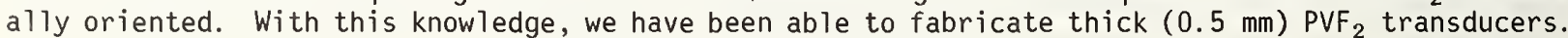

\section{Nondestructive Evaluation}

\section{S. Edelman, and S. C. Roth}

A method for nondestructive evaluation and the dynamic elastic properties of some kinds of structural elements was worked out during previous years, and a paper on the subject was presented at the Spring 1980 meeting of the Acoustical Society of America. The measurement technique made use of the favorable properties of piezoelectric polymer strain gauges to measure both axial and shear motion on the same specimen. The specimen can be evaluated nondestructively while it is in service. This subtask demonstrates to potential manufacturers this important application of piezoelectric polymers and is supported by the NDE program at NBS.

During this reporting period, a set of the specialized piezoelectric strain gauges required by the method was fabricated. The gauges were mounted on a thin-walled PVC cylinder which simulated a structural element under load.

Several measurements were made at different frequencies. The real and the imaginary parts of the dynamic Young's modulus were calculated. Reasonable agreement with published values of the real part was found. The results of two sets of measurements at slightly different frequencies are given in the table below. Each set consists of three nominally identical measurements.

\begin{tabular}{ccccc} 
Frequency & $\begin{array}{c}\alpha \\
\left(\mathrm{m}^{-1}\right)\end{array}$ & $\underline{\left(\mathrm{m}^{-1}\right)}$ & $\begin{array}{c}\mathrm{E}_{1} \\
(\mathrm{~Pa})\end{array}$ & $\begin{array}{c}\mathrm{E}_{2} \\
(\mathrm{~Pa})\end{array}$ \\
\cline { 5 - 5 } 6915 & 3.19 & 27.33 & $3.37 \times 10^{9}$ & $7.97 \times 10^{8}$ \\
& 3.19 & 27.32 & $3.37 \times 10^{9}$ & $7.99 \times 10^{8}$ \\
& 3.19 & 27.33 & $3.37 \times 10^{9}$ & $7.99 \times 10^{8}$ \\
6836 & 2.82 & 27.36 & $3.32 \times 10^{9}$ & $6.91 \times 10^{8}$ \\
& 2.82 & 27.38 & $3.31 \times 10^{9}$ & $6.92 \times 10^{8}$ \\
& 2.83 & 27.38 & $3.29 \times 10^{9}$ & $7.45 \times 10^{8}$
\end{tabular}

$\alpha$ and $\beta$ are the attenuation and phase constants which make up the propagation constant $\gamma$ where $\gamma=\alpha+$ $\beta$ and where $\gamma$ is related to the wave amplitude $\xi_{x}$ by the relation $\xi_{x}=\xi_{0} e^{-\gamma x}$.

$E_{1}$ and $E_{2}$ are the real and imaginary parts of the Young's modulus, $E=E_{1}+i E_{2}$.

Measurements are presently being made on a section of pipe while the pipe is subjected to a changing tensile stress.

\section{ASTM Activities}

\section{W. P. Harris}

This involvement in ASTM is one method by which the task output impacts the industry. Mr. Harris attended the 0ctober 1980 and March 1981 meetings of ASTM Committee D-9, Electrical Insulating Materials. He continues to be active in several subcommittees, especially in the area of electrical measurements. He is also active on the Executive Subcommittee, which is responsible for governing the main Committee. 
The Crystal Structure and Disorders of the Low Temperature Phase (II) of Polytetrafluoroethylene

\author{
J. J. Weeks, E. S. Clark ${ }^{1}$, R. K. Eby, B. L. Farmer ${ }^{2}$
}

Long-term durability studies of a series of polymers revealed that fluoropolymers have superior stability of dielectric and mechanical properties. Therefore, they were chosen to make standard reference materials for calibration of industrial dielectric cells. As noted below, this has indeed turned out to be the case. To investigate the origin of this superior stability, we have carried out studies of the crystal structure.

An electron diffraction pattern from polytetrafluoroethylene in Phase II was analyzed to determine a chain conformation of $2.1598 \mathrm{CF}_{2}$ units per turn of the helix. A structure was established from a combination of electron and $x$-ray diffraction data. The structure contains an ordered repeating pattern of a left- and right-handed pair of chain stems. Although the structure is ordered in three dimensions, it cannot be described meaningfully in terms of classical space group nomenclature because very small changes in the structure result in very large changes in the cell parameters. The structure has a volume of $0.03542 \mathrm{~nm}^{3}$ per $\mathrm{CF}_{2}$ group, corresponding to a density of $2344 \mathrm{~kg} / \mathrm{m}^{3}$.

Energy analysis was used to determine the low energy crystal structure for polytetrafluoroethylene molecules in the $13 / 6$ and $54 / 25$ conformations. The structure for the $54 / 25$ conformation contains an ordered repeating pattern of a left- and right-handed pair of molecules in a unit cell having projected parameters, $a^{\prime}, b^{\prime}$, and $\gamma^{\prime}$, of $9.60 \AA, 5.62 \AA$, and 91.4 degrees, respectively. These results, the relative orientations of the molecules, and the relative translations of the molecules are in good agreement with results obtained in a concurrent and independent analysis of diffraction data. Energies calculated for rotational and translational displacements in various modes suggest that a range of structures and a fairly large anount of disorder may be present, also in agreement with experiment.

$X$-ray diffraction patterns for oriented and unoriented copolymers of tetrafluoroethylene and hexafluoropropylene were obtained. They show a transition between 229 and $243 \mathrm{~K}$ for a copolymer with about $3.4 \mathrm{CF}_{3}$ per hundred main chain carbon atoms. The lower temperature phase (II) appears to be similar to that of the tetrafluoroethylene homopolymer. A primary difference is the presence in the copolymer crystals of small longitudinal displacements of the molecules caused by the randomly located $\mathrm{CF}_{3}$ groups. The displacements increase with increasing $\mathrm{CF}_{3}$ concentration. The higher temperature phase (I) is similar to that of the homopolymer with a difference again being the longitudinal disorder of the copolymer. The difference disappears at higher temperatures as longitudinal disorder is introduced thermally in the homopolymer. At $296 \mathrm{~K}$ (phase I), the molecular stems in a copolymer with about $3.4 \mathrm{CF}_{3}$ per hundred main chain carbon atoms are at an angle of 37.1 degrees with respect to the normal to the basal plane of the lamellas. In phase II, this number is the same within the limits of error.

\title{
MIGRATION AND THE DURABILITY OF POLYMERS IN USE
}

\section{E. Smith}

\section{Task Leader}

The most promising areas of growth in polymer markets are in construction, transportation, and electrical systems where long term durability is essential to competitive performance. Increased awareness of the problems of productivity and heightened competition has intensified this concern with durability in all polymer materials.

Reliable test methods that reflect long term durability are needed for the selection of appropriate polymers for specific uses or to maximize industrial productivity by reducing overdesign of parts without inducing premature failure. Reliable methods for the prediction of the lifetime of polymers in service rely on three separate types of information:

(1) Knowledge of the rates and mechanisms by which the polymers degrade under the appropriate environmental conditions.

(2) Information concerning the rates of diffusion of protective additives; (Almost all commercial polymers are too sensitive to environmental attack to be used without protective additives. These low molecular weight substances such as antioxidants or stabilizers inhibit the degradation reaction and are dispersed throughoutte polymer to protect it. In use, these additives migrate through the polymer leaving the polymer markedly more vulnerable to failure.)

(3) Relationships connecting the changes in molecular properties to the commercially useful properties of the polymers.

Work in this task is concerned with all three of these needs. Item (3) is addressed in both this task and in task 12135, Mechanical Durability of Polymers.

\footnotetext{
${ }^{1}$ Consultant, University of Tennessee

"Consultant, Washington State University
} 
Technical work this year on degradation mechanisms and rates includes investigations of the hydrolys is of polyesters, a study of the mechanism of oxidation of polystyrene, the use of thermogravimetry to measure the rates and activation energies of the degradation of several polymers, and analysis of thermal methods of lifetime prediction.

For most commercial polymers, lifetime prediction methods must explicitly include the rates of additive diffusion along with the rates of the inhibited and uninhibited degradation reactions. The number of commercially usable polymer-additive combinations is too large to be considered eficiently on a case-by-case basis. The emphasis in this task is, therefore, on the development of general principles or models of diffusion that are applicable to the maximum number of commercially important systems. In addition, knowledge of the general principles which control diffusion through solid polymers is important to the design and evaluation of improved additive systems.

Polyolefins, which include psolyethylene and polypropylene, account for approximately half of the total polymer production. For this reason, the work in this task on models for diffusion is concerned exclusively with polyolefins which have their amorphous regions above their glass transition temperatures in normal use conditions. We have developed a useful empirical correlation equation which relates the diffusion constants among a large number of polymer-additive pairs. Current work is now focused on the investigation of rapid and convenient methods for the measurement of diffusion rates by solvent extraction and inverses chromatography and on the accurate measurement of slow diffusion rates in solid polymers by microfluorimetry. These accurate measurements will provide improved data with which refined correlations can be constructed. These refined correlations should lead to theoretical descriptions of diffusion based on the molecular structure of the additives and the structure and morphology of the polymers.

\section{Hydrolytic Degradation of Polyester Polyurethane Elastomers}

\section{W. Brown, R. E. Lowry, L. E. Smith}

Polyester polyurethanes are versatile specialty polymers which are used to form tough and durable cast or molded elastomeric parts such as oil seals, gaskets, and electrical connectors. They are frequently used in critical and demanding service where the lifetime and performance of major systems depend on their durability. Some examples include aircraft engines, ship propulsion assemblies, submarine oxygen generators, and nuclear reactor control rod actuators. In order to provide reliable lifetime prediction methods for such applications, we have measured the rates of chain scission of unstabilized polyester polyurethanes. Activation energies for the degradation were measured to enable the use of accelerated test methods. We then devised a rapid and convenient measurement method which uses the acid contents of the polymers to predict degradation rates in service.

During this year we have measured the rates of the inhibited reaction to evaluate the effect of stabilizers on the expected lifetime and have produced a test method which gives results that correlate well with field experience.

We have recently described the hydrolytic breakdown of carbodiimide-stabilized polyester polyurethanes [1]. Polyester degradation ordinarily occurs by an acid catalyzed hydrolysis of the ester linkages. Carbodiimide stabilizes the polymer by reacting with acid, thus preventing catalysis. We have shown that uncatalyzed hydrolysis of ester also occurs. This reaction produces acid at a slow rate which reacts with the carbodiimide, eventually consuming it. Thereafter, acid catalysis again becomes important. Consequently, there were three rate constants to consider: $k_{0}$ for uncatalyzed hydrolysis, $k$ for acid catalyzed hydrolysis, and $k^{\prime}$ for reaction of acid with carbodiimide. These were evaluated separately from the slow disappearance of carbodiimide, polymer hydrolysis without stabilizers, and reaction of polyester acid and carbodiimide in a dry polyester polyurethane, respectively. Differential equations for the combined reactions were then numerically integrated to generate curves that show the time dependence of the acid concentration, [A], the carbodiimide concentration, [M], and the change in the reciprocal of the number average molecular weight, $\Delta\left(M_{n}^{-1}\right)$. This last is equal to the number of chain scissions. The figure shows one set of curves and the corresponding experimental data for stabilized and unstabilized polymers. The increase in service life due to the presence of carbodiimide is approximately equal to $\left(\left[M_{0}\right]-\left[A_{0}\right]\right) / k_{0}$, where the subscripts indicate use of concentrations measured after incorporation of the carbodiimide in the polymer. Values of $k_{0}$ depend on temperature but are the same for polycaprolactone and polybutylene adipate based polyurethanes of laboratory and commercial origin. Consequently, the $k_{0}$ should be generally useful in estimating lifetimes of stabilized polyurethanes.

Two aspects of the hydrolysis are still being studied. One is the dependence of $k_{0}$ on the urethane content of the polymer, since $k_{0}$ of polyester diols is three- to ten-fold less than $k_{0}$ of the corresponding polyurethanes. The other concerns the position of the ester-acid equilibrium in polyester diols and polyurethanes. This equilibrium would define an optimum environmental condition where no further hydrolysis would occur. This has important implications for archival uses of polyesters for information storage and retrieval. 
Reference:

[1] D. W. Brown, R. E. Lowry, and L. E. Smith, The Hydrolytic Degradation of Polyester Polyurethanes Containing Carbodi imides, submitted to Macromolecules.

Long Term Aging Studies With Poly(Ethylene Terephthalate)

\author{
D. W. Brown, R. E. Lowry, L. E. Smith
}

Polyesters are the base material of a number of information storage and retrieval systems, such as photographic film, magnetic video and audio recording tape, and electrographic record systems. These storage media are replacing paper books and records even in archival storage because of their much higher information densities. In a project sponsored by the National Archives, we are investigating methods for estimating the useful lifetimes of polyesters and attempting to define optimum storage conditions for these materials.

Hydrolysis of the ester linkage is the most important cause of degradation. Thus, the general features of the degradation of the polyester polyurethanes are expected to be followed with complications caused by the higher glass temperature $\left(\sim 70{ }^{\circ} \mathrm{C}\right)$, the presence of crystallinity, and the extreme orientation needed to make a strong film.

Experiments that have been set up include agings in air at 0, 25, 50, and 100 percent relative humidity $(\mathrm{RH})$ at temperatures of 35,55 , and $85^{\circ} \mathrm{C}$. One set of samples at 100 percent relative humidity is in sealed tubes under nitrogen. Amorphous, unoriented samples are also being aged. Samples include a variety of commercial record-keeping films as well as samples of the NBS SRM 1470, a polyester film. Measurements being made on the samples include tensile tests, acid analysis by titration, molecular weight measurements by gel permeation chromatography, and examination by differenital scanning calorimetry.

To date, we have found that 90 days aging of SRM 1470 at 100 percent RH at $85{ }^{\circ} \mathrm{C}$ causes $\sim 90$ percent loss in tensile strength and a reduction in the number average molecular weight from 14500 to 6300. A sample aged at 100 percent $\mathrm{RH}$ at $55{ }^{\circ} \mathrm{C}$ for 90 days lost negligible tensile strength, and its molecular weight was 12600 . Calorimeter scans showed no significant effects on the main melting peak $\left(\sim 255^{\circ} \mathrm{C}\right)$ compared with the original material, but small peaks were introduced near the aging temperature. Very severe degradation resulting in weak, brittle samples occurred at 100 percent RH in 13 days at $115^{\circ} \mathrm{C}$ and in 39 days at $100{ }^{\circ} \mathrm{C}$, molecular weights being $\sim 2800$ and $\sim 3100$, respectively. With these samples, the melting peak was much broadened, with maxima occurring at 243 and $249{ }^{\circ} \mathrm{C}$, respectively. Peak areas were increased about 20 percent.

It is planned to take advantage of the autocatalytic effect by preaging samples at $85{ }^{\circ} \mathrm{C}$, 100 percent $\mathrm{RH}$ and then aging the samples under milder conditions, where the high acid content will result in more rapid changes.

\title{
Oxidation of Polystyrene
}

\section{B. Dickens}

Polystyrene is a high volume commodity polymer subject to oxidation in use. The mechanism of oxidation under normal use conditions is not known with certainty. Studies carried out in collaboration with Dr. Jean Marchal of the CNRS, Strasbourg, have used $\gamma$-ray initiated oxidation of polystyrene to simulate the low temperature oxidation process encountered under normal use but with control over the rate of radical initiation.

0xidation studies were carried out on polystyrene in chlorinated hydrocarbon solutions to produce readily radicals which are capable of attacking the polymers. Product analysis coupled with measurements of molecular weight changes have led to a number of conclusions. Under these circumstances, we conclude that: the initial attacking species are $C l$ radicals, attack is random along the PS chain, intramolecular propagation of the oxidation chain occurs by hydrogen abstraction by peroxyl radicals, on the average there is one such propagation step on the PS backbone in $\mathrm{CHCl}_{3}$ and $\mathrm{six}$ in $\mathrm{CCl}_{4}, \mathrm{higher}$ molecular weights are attacked less per monomer unit than lower molecular weights, and alkoxyl radicals are formed and undergo $\beta$-scission to break the polymer backbone and produce small molecules. In the solid these small molecules would plasticize the material and impair its useful mechanical properties. An oxidation mechanism to account for these facts has been devised.

\section{Variable Heating Rate Thermogravimetry}

$$
\text { J. H. Flynn }
$$

The concept of measuring the degradation of a polymeric material under conditions designed to accelerate its rate and extrapolating the rates to milder conditions so as to predict the lifetime of the material has great appeal. The practical uses and commercial importance of reasonably accurate 


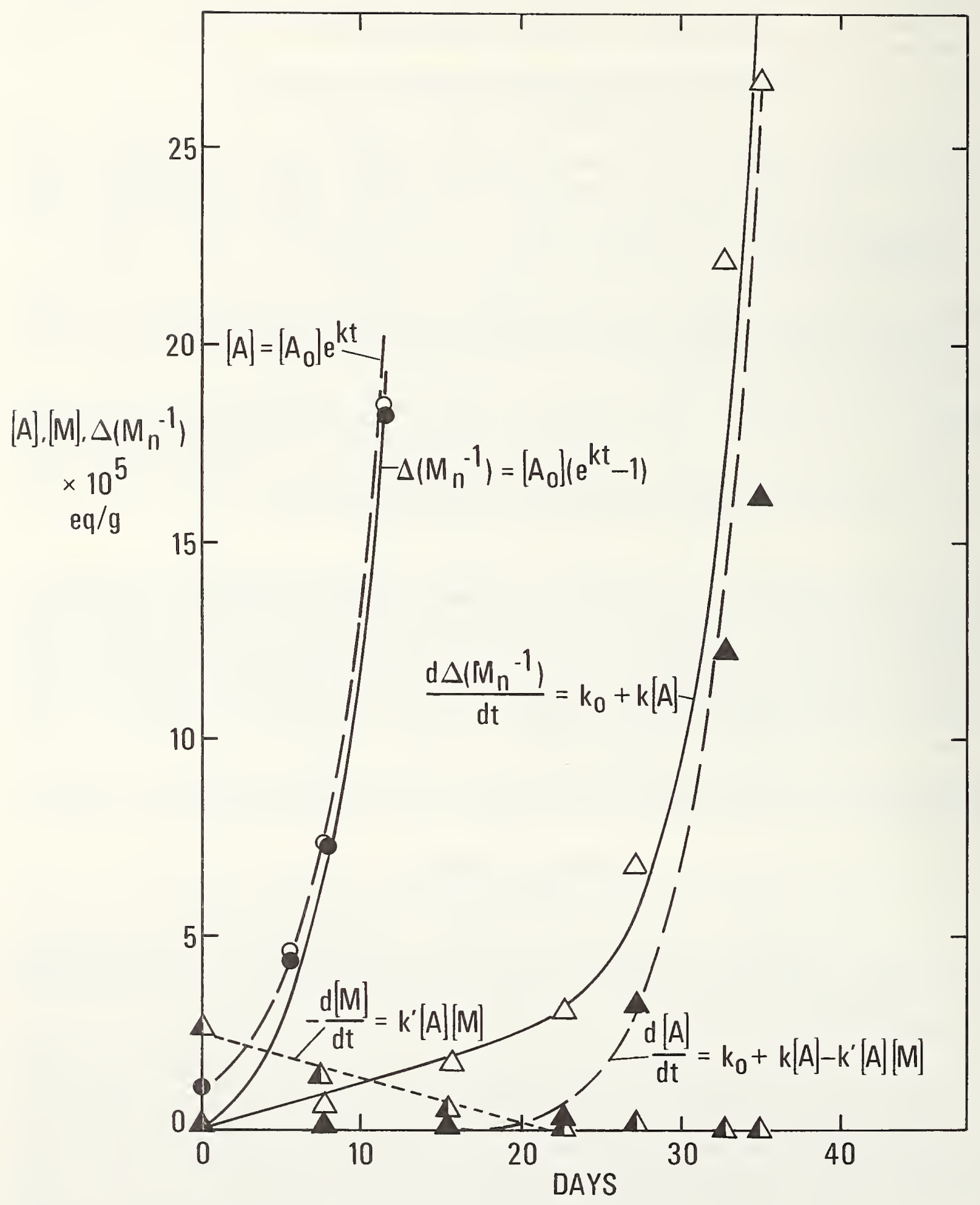

Figure 1. Hydrolytic degradation of a polybutylene adipate-based polyurethane at $85{ }^{\circ} \mathrm{C}$, 100 percent relative humidity: with carbodi imide, triangles; without carbodi imide, circles, [A] shaded, [M] half-shaded $\left(\Delta M_{n}^{-1}\right)$ unshaded. Lines with $k_{0}=1.2 \times 10^{-6}$ equivalent $/ \mathrm{g} \cdot$ day, $k=0.26$ day $^{-1}, n_{\text {and }} k^{\prime}=3.0 \times 10^{5} \mathrm{~g} / \mathrm{eq} \cdot$ day. 
lifetime prediction methods are obvious. The methods of thermal analysis are often used in lifetifis prediction procedures. They offer practical advantages such as accessibility of apparatus, simplicity of application, and rapid accumulation of data. Thermogravimetry is the method of thermal analysis most often used for this purpose because the kinetics of volatilization are often closely coupled to the kinetics of degradation in the condensed phase.

Unfortunately, the application of the Arrhenius equation to polymer degradation processes for the purpose of predicting aging at service conditions is complicated by the complex kinetics of these reactions. Such heterogeneous systems are often composed of at least several "elementary processes," each with its own set of Arrhenius parameters. A change in activation energy will result from a change in the rate-limiting step of the degradation mechanism. If this occurs in the unmonitored kinetic gap between the lowest experimental temperature and the service temperature, then the lifetime prediction is invalid.

In our variable heating rate method, kinetic data are compared over a thousand-fold range of heating rates--from six degrees/minute to nine degrees/day. As a result, activation energies are obtained from five to 95 percent conversion for over a hundred degree temperature range and down to much lower temperatures than those of the conventional methods of thermal analysis. From isoconversional diagnostic plots of logarithm heating rate vs reciprocal absolute temperature, activation energies are calculated for each fraction of conversion. The wide range of temperatures which these measurements cover gives one the precision necessary to determine the activation energies from the slope between each successive pair of points on each isoconversional. A grid may be set up from these values in which activation energies (E) are displayed as a function of conversion and temperature. This new technique has been applied to the degradation kinetics of polystyrene, poly(methylmethacrylate), and polyurethanes in inert atmosphere and in air. In the case of poly(methylmethacrylate) in nitrogen, for example, activation energy grids demonstrate that $E$ increases with increasing temperature at constant conversion, but $E$ appears to be nearly independent of conversion at constant temperature.

\section{Analysis of the Kinetics of Thermogravimetry}

\section{J. H. Flynn}

The kinetic analysis of the thermogravimetry of polymers requires the comparison of data from experiments performed at differing temperature programs. These data reflect divergent physical properties and chemical compositions among the specimens resulting from their diverse thermal histories. The effect of thermal history on the kinetics of weight-loss processes is a serious problem. However, it is not usually dealt with during the modeling of the kinetics of these processes. A reason for this is that often the effect can be exposed only through the comparison of calculated parameters from two separate series of kinetic measurements on identical specimens of polymers. 0therwise, contradictions in values of kinetic parameters from different techniques and investigations may either be overlooked or falsely ascribed to other causes such as experimental error, differences in initial chemical or physical properties of the samples, differences in experimental conditions, etc.

The critical test for thermal history dependence is differing rates of weight loss from two experiments at the same temperature and conversion, i.e., at a point of concurrence. The conventional methods for the determination of Arrhenius parameters involve either the comparison of two or more isothermal experiments at differing temperatures or the comparison of two or more constant heating rate experiments at differing heating rates. Neither of these cases realize a point of concurrence between two experiments.

Two experimental techniques and methods of data analysis have been developed to detect thermal history dependence of Arrhenius parameters. In the first method, logarithms of the times to reach various fractions of conversion for several isothermal experiments are plotted against the reciprocal absolute temperatures at which the same fractions of conversion were reached in a single experiment at constant heating rate. The slopes of the isothermals are given by:

$$
\text { isothermal slope } \sim-1.05 E R^{-1}
$$

and the isoconversional distance between two isothermals, $T_{a}$ and $T_{b}$, is

$$
\text { isothermal distance }=E R^{-1}\left(T_{b}^{-1}-T_{a}^{-1}\right) \text {. }
$$

If thermal history effects are present, then the activation energy (E) calculated from the slopes will differ significantly from E calculated from the isothermal distances.

Similar sets of equations have been developed in the second case in which a single isothermal experiment is compared with several experiments at various constant heating rates. For this case, E's obtained from the isothermal distance between two isothermokinetics are compared with those obtained from the slopes of the isothermokinetic lines. As before, any difference between $E$ calculated from the slopes and E calculated from the distances will indicate thermal history dependence. Also, in 
either case, any deviation of $E$ from constant value can be observed visually from a distortion of the uniform distances or from changes in slope of the grids.

Activation energies have been determined with great precision for the degradation of polystyrene in vacuum from both the factor jump and constant heating rate techniques. The $E$ values from the two methods show a significant difference of $11.1 \mathrm{~kJ} / \mathrm{mol}$. This difference has been interpreted as due to a bias from a constant increment caused by different thermal histories for each rate of heating.

\section{Thermogravimetric Studies of Polymer Degradation}

\section{B. Dickens}

Because of its ease of application, thermogravimetry is widely used to study polymers. However, this ease of application also induces a significant amount of misapplication. The appropriate kinetic treatments for thermogravimetric data have been examined and reviewed in a joint publication with J. H. Flynn. The parameter most readily determined is the activation energy, which can then be used to shed some light on the degradation mechanism.

In recent degradation studies, the following activation energies were found: $65.4 \pm 0.5 \mathrm{kcal} / \mathrm{mol}$ for both linear and branched polyethylene (LPE and BPE) degrading in vacuum, 64.8 $\pm 0.3 \mathrm{kcal} / \mathrm{mol}$ for LPE and $64.4 \pm 0.2 \mathrm{kcal} / \mathrm{mol}$ for BPE in slowly flowing $\mathrm{N}_{2}$ at $8.0 \mathrm{~mm} \mathrm{Hg}$, and $6.26 \pm 0.5 \mathrm{kcal} / \mathrm{mol} \mathrm{for} \mathrm{LPE}$ and $61.2 \pm 0.6 \mathrm{kcal} / \mathrm{mol}$ for $\mathrm{BPE}$ in $\mathrm{N}_{2}$ flowing at 1.0 to $4.0 \mathrm{~mm} / \mathrm{s}$ at $800 \mathrm{~mm} \mathrm{Hg}$. The overall activation energy in vacuum has been related to a degradation mechanism to show that the slow chain-breaking step is one of $\beta$-scission following hydrogen abstraction. The procedure provides an estimate of $33 \mathrm{kcal} / \mathrm{mol}$ for the activation energy of $\beta$-scission for secondary alky 1 radicals. For degradation by random scission of $C-C$ backbone bonds in the initial stage, the overall activation energy is estimated to be $70.6 \mathrm{kcal} / \mathrm{mol}$. The lower activation energies in the presence of an external pressure have been shown to be due to the evaporation of molecules formed in scissions but not volatilized immediately after their formation. This adds a latent heat term (10 to $50 \mathrm{kcal} / \mathrm{mol})$ to the apparent activation energy.

Similar quantities have been determined for isotactic polypropylene: $61.5 \pm 0.8 \mathrm{kcal} / \mathrm{mol}$ in vacuum, $55.9 \pm 0.3 \mathrm{kcal} / \mathrm{mol}$ in $\mathrm{N}_{2}$ at $5.0 \mathrm{~mm} \mathrm{Hg}$, and $55.1 \pm 0.2 \mathrm{kcal} / \mathrm{mol}$ in $\mathrm{N}_{2}$ flowing at $1.0 \mathrm{to} 4.0 \mathrm{~mm} /$ $s$ at $800 \mathrm{~mm} \mathrm{Hg}$. The decomposition of the activation energy into its components for degradation in vacuum using a degradation mechanism devised to be consistent with the observed degradation behavior and degradation products provides an estimate of $29.6 \mathrm{kcal} / \mathrm{mol}$ for the $\beta$-scission of tertiary alkyl radicals. As was the case for $P E$, the lower activation energy for degradation under external pressure has been shown to be due to the latent heat contributions of molecules which do not evaporate as soon as they are formed. For the initial stage of degradation in vacuum, an activation energy of 71 kcal/ mol is proposed.

\section{Measurement of Antioxidant Diffusion by Microfluorimetry}

\section{S. L. Wunder, F. W. Wang, and R. A. Velapoldi ${ }^{1}$}

Measurement of the concentration profiles of a diffusion substance is an accurate method for determining relatively small diffusion constants in the solid state. In addition, the effect of solvent on the diffusion rate can be measured on the same system which will allow direct comparison with diffusion rates measured by rapid and convenient solvent extraction methods.

The migration of antioxidants from polymer films is being studied by both extraction experiments and solid state diffusion experiments. In an extraction experiment, a plan sheet of polymer with uniform concentration of an antioxidant is immersed in a limited amount of well-stirred solvent. Then, the amount of the antioxidant extracted by the solvent is determined at various times by fluorimetry. The extraction of $N, N^{\prime}$-diphenyl-p-phenylendiamine (DPPD) by heptane at $22.0^{\circ} \mathrm{C}$ from $10 \mathrm{w}$ density polyethylene films has been measured. An analytical working curve relating DPPD concentration to fluorescence intensity was linear in DPPD concentration below $4.0 \mathrm{ppm}$. A preliminary report of the results has been prepared.

The diffusion of the antioxidant $N, N^{\prime}-\beta$-dinaphthyl-p-phenylene-diamine (DNPD) in branched polyethylene (PE) SRM 1476 is being studied using microfluorimetry. After fusion of two plugs, one neat $P E$ and the other doped with antioxidant, measurement of the antioxidant fluorescence as a function of distance from the fused boundary yields the diffusion coefficient of the antioxidant. This solid state diffusion technique eliminates the problem of the swelling effects in extraction experiments. Preliminary work has been performed to optimize the experimental conditions and techniques for the diffusion measurements. These will be obtained for DNPD in PE as a function of temperature. 


\section{Migration of 01 igomers and Antioxidants From Polyolefins}

\section{S. S. Chang, W. J. Pummer, J. R. Maurey, L. E. Smith}

Under the sponsorship of the Food and Drug Administration, this project aims at the development of general principles and models of diffusion that can be used to simplify the regulations governing polymers in food contact use and thus ease the burden of Federal regulations necessary for public health and safety. This work is also directly applicable to the design and interpretation of extraction methods for diffusion measurement and the models developed will be applicable to the problem of lifetime prediction as well. In this year, copolymers of ethylene and vinyl acetate with vinyl acetate contents of five and 13 percent have been added to the list of polyolefins studied. Several solvents have also been added to the list of solvents used.

Thus, the experimental variables include (1) polymers--linear polyethylene, branched polyethylene, isotactic polypropylene, and copolymers of ethylene and vinyl acetate; (2) migrants--n- $\mathrm{C}_{18} \mathrm{H}_{38}$, $n-\mathrm{C}_{32} \mathrm{H}_{66}$, and BHT; (3) solvents--corn oil, tributyrin, trioctanoin, ethanol, ethanol-water mixtures, water, n-heptane, n-octanol, synthetic triglyceride mixture HB307, and n-octadecane; and (4) temperature--30, 40, and $60^{\circ} \mathrm{C}$. Furthermore, the initial concentrations of the migrants in the polymers and the thickness of the sample plaques have also been varied. Besides the kinetic studies, equilibrium properties such as solubilities and partition coefficients have also been studied. The migrants used are labeled with radioactive ${ }^{14} \mathrm{C}$ to yield a detectibility of $10^{-10} \mathrm{~g}$ if needed. The extraction experiments generally require a few weeks to a few months to complete; however, some experiments may reach completion in less than a day, while some may last longer than a year. The diffusion coefficients observed vary from $10^{-6}-10^{-15} \mathrm{~cm}^{2} \mathrm{~s}^{-1}$. Some of the experiments indicate an ideal Fickian behavior, especially for preswollen polymers or for non-swelling solvents. In most solvents, the diffusion coefficient may increase from an initial value and then level off at a higher value due mainly to swelling effects.

If all other parameters are held constant, then the ranking of importance of the influence of parameters on the diffusion coefficient of migrants from polyolefins is as follows: solvent, temperature, additive type, polymer type, additive concentration. The first three parameters are far more important than the remaining two.

The nature of the effect of the first three variables is also rather different from those of the latter. The effect of the most influential group of variables (solvent, temperature, and migrant) is generally much less pronounced for cases producing high diffusion coefficients, e.g., $10^{-6} \mathrm{~cm}^{2} \mathrm{~s}^{-1}$, than for low diffusion coefficients. For instance, the effect of replacing a lower MW oligomeric migrant, $n-C_{18} \mathrm{H}_{38}$, by a higher MW migrant, $n-\mathrm{C}_{32} \mathrm{H}_{66}$, under various conditions is shown in figure 2 as an illustration for this type of behavior. The effect is less than a factor of five at diffusion coefficients of $10^{-7}$ to $10^{-6} \mathrm{~cm}^{2} \mathrm{~s}^{-1}$, while the ratio is near 1,000 at diffusion coefficients of $10^{-10}$ to $10^{-9} \mathrm{~cm}^{2} \mathrm{~s}^{-1}$. Similar behavior is observed by changing solvents or temperature.

However, in the case of changing polymers, the effect seems to be more or less a constant shift in the ratio of the diffusion coefficients covering a wide range of diffusion coefficients. This behavior is illustrated in figure 3 where the diffusion coefficients of migrants from low crystallinity, branched polyethylene are compared against those from highly crystalline, linear polyethylene (as well as polypropylene) in various combinations of other parameters. The average ratio of the coefficients for changing the base polymer only is about six through the range of diffusion coefficients from $10^{-14}$ to $10^{-6} \mathrm{~cm}^{2} \mathrm{~s}^{-1}$.

In contrast to the great accelerating action of n-heptane over that of corn oil in extracting small molecules from polymers (NBSIR 81-2263), the diffusion coefficients of these small molecules into liquidus tributyrin, trioctanoin, and synthetic triglyceride HB307 are nearly identical to that into corn oil within a factor of 1.5. Therefore, either tributyrin, trioctanoin, or HB307 can be used to simulate the extractive action of corn oil in a great variety of conditions involving different additives, polyolefins, and temperatures. However, normal analytical techniques (other than radiolabeling) are difficult with the triglycerides. Diffusion coefficients into ethanol are equal or slightly greater than that into triglycerides under similar combinations of test conditions and chemical analysis in ethanol is generally much easier than in the triglycerides. The slightly enhanced extraction behavior makes ethanol a slightly accelerating solvent which yields an upper bound for migration.

Results for n-octanol and 95 percent ethanol yield supplemental information for using ethanol as a simulating solvent. Octanol also behaves as an accelerating solvent, although its accelerating action is far below that of n-octadecane and n-heptane. Diffusion coefficients of these small molecules are about the same into pure ethanol and into 95 percent ethanol. However, the lower solubility and partition coefficients of some of the migrants in 95 percent ethanol yield a lower limit of migration of these molecules at equilibrium than that in anhydrous ethanol. 


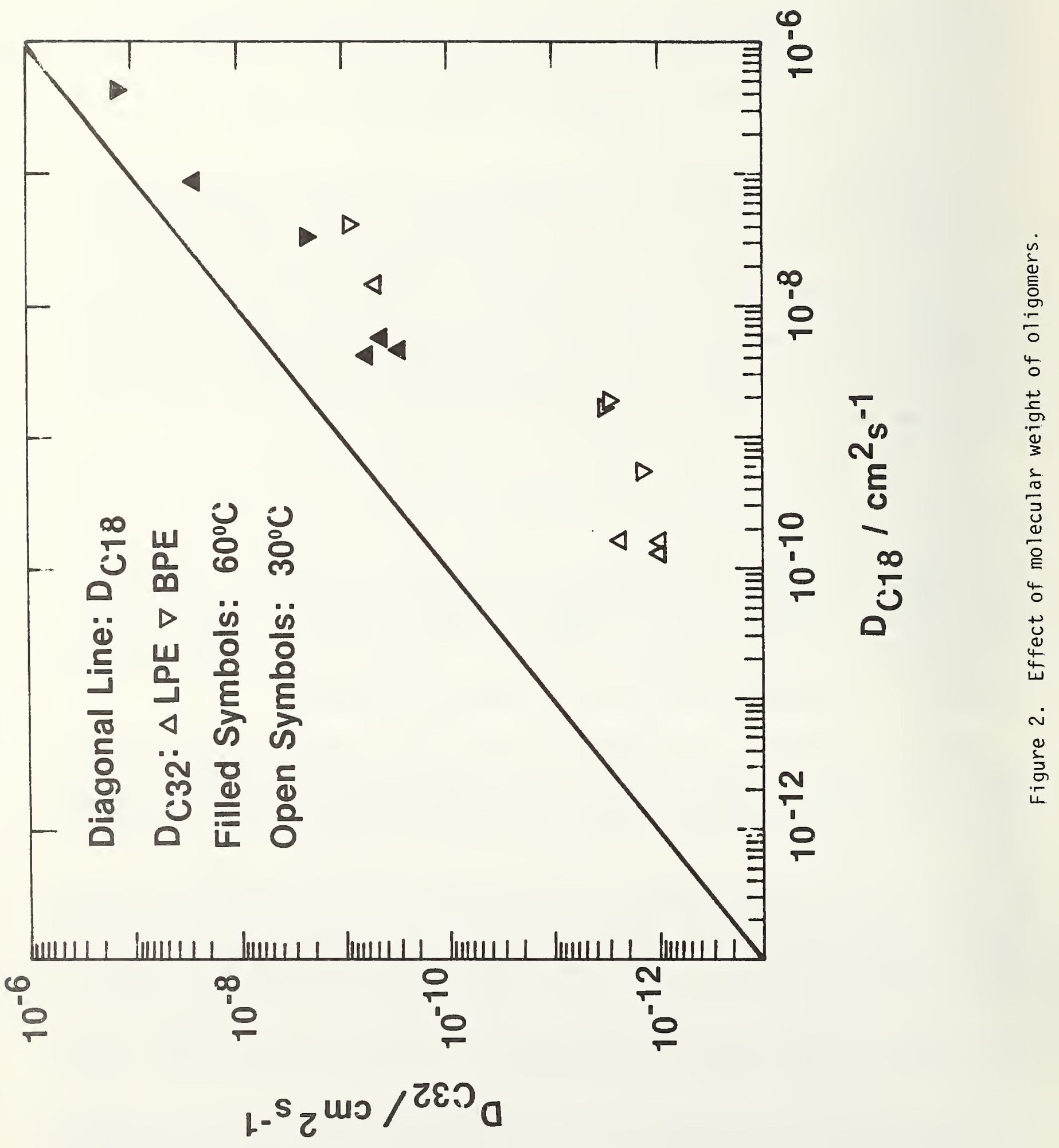




\section{G. A. Senich}

Diffusivities of volatile compounds or probes in polymers have been obtained from non-equilibrium inverse gas chromatography (IGC) experiments conducted at rapid carrier gas flow rates. The experiment is similar to conventional gas chromatography (GC) except that the polymer under study replaces the usual low molecular weight stationary phase. The quantity of interest is the measured peak broadening that occurs during flow through the column. Peak spreading arises from the multiplicity of differing gas paths through the column, diffusion in the gas phase along the flow direction, and resistance to mass transfer in both the gas and polymer phases, among other factors, and is commonly related to the probe-polymer diffusion coefficient by the well-known van Deemter equation in GC. However, this relation neglects variations in the local carrier gas flow velocity and some additional diffusive processes in the gas phase. These factors have been shown to exert a significant influence on the magnitude of the diffusivity found by IGC studies.

Another important parameter is the diffusion path length in the polymer phase. Two extremes in polymer geometry are possible within the column, a thin, uniformly distributed covering over the entire surface of the spherical column packing or annulus-like regions collected at the contact points between spheres, each with a different diffusion path length. These two extremes in the physical distribution of the polymer phase can lead to polymer-probe diffusivities differing by factors of up to ten thousand. The variability of the polymer film throughout the column makes an accurate determination of the absolute probe-polymer diffusivity unlikely. Experiments on the rate of diffusion of one probe relative to another in the same polymer column are currently in progress. The resulting relative diffusivities may then be placed on an absolute scale by calibrating the column with a probe whose diffusion coefficient in the polymer has been determined by an independent method.

The extent of migration of a sufficiently volatile material from a polymer into a contacting substance (typically a solvent) can be estimated from data readily obtained by IGC studies. The specific retnetion volume obtained experimentally at slow carrier gas flow rates, which characterizes the thermodynamic interactions between the probe and polymer, is used to determine the polymer-migrant interaction parameter. When combined with a value of the solubility of the migrant in the solvent, a calculation of the equilibrium partition coefficient is possible. The latter is a direct measure of the fraction of the additive which can be expected to migrate from the polymer to the contacting medium. If the time over which migration can occur is short, an equilibrium prediction may greatly exaggerate the degree of migration expected. A value for the diffusion coefficient of the migrant in the polymer may then be obtained from IGC experiments and employed to estimate the degree of migration which will occur over the given time period. A recent publication [1] outlines in greater detail a procedure for determining the extent of migration at both equilibrium and short times from data derived by IGC studies.

Reference:

[1] G. A. Senich, Chem. Tech. 11, 360 (1981).

Measurement of Translational Diffusion of Polymers by Fluorescence Photobleaching Recovery Technique

F. W. Wang, and W. H. Grant

The diffusion coefficient of chain molecules is a dynamical parameter which should provide a test of both scaling [1] and reptation [2] concepts proposed by de Gennes. Recently, Hervet and co-workers [3] used the forced Rayleigh scattering technique to measure the diffusion coefficient (D) of polystyrene chains in benzene. They found that in the semidilute regime where the chains overlap, $D$ decreases with polymer concentration, $c$, as $c^{1} 701$, in agreement with scaling law predictions. However, they pointed out that more experiments are necessary to check the molecular weight dependence of the diffusion coefficient.

In collaboration with Prof. En-Shinn Wu of the University of Maryland (Baltimore County), we have initiated a project to measure by the fluorescence photobleaching recovery (FPR) technique the diffusion of chain molecules in the semidilute regime. In the FPR technique, one uses high intensity illumination to deplete the observed volume element of chromophore-labeled chain molecules by photobleaching. After the bleaching pulse, the diffusion of unbleached chromophore-labeled chain molecules into the volume element is observed with low intensity illumination. From the time constant for the recovery of the original fluorescence intensity, the diffusion coefficient can be obtained. The FPR technique can be used to measure diffusion coefficients in the range $10^{11} \mathrm{~cm}^{2} / \mathrm{s} \mathrm{to} 10{ }^{6} \mathrm{~cm}^{2} / \mathrm{s}[4]$.

We have developed a procedure to synthesize polymers labeled with photobleachable chromophores and have determined the fluorescence properties of one of these polymers. The diffusion coefficients of this and other similarly prepared polymers will be measured by using the FPR technique to check the molecular weight dependence of the diffusion coefficient in the semidilute regime. 


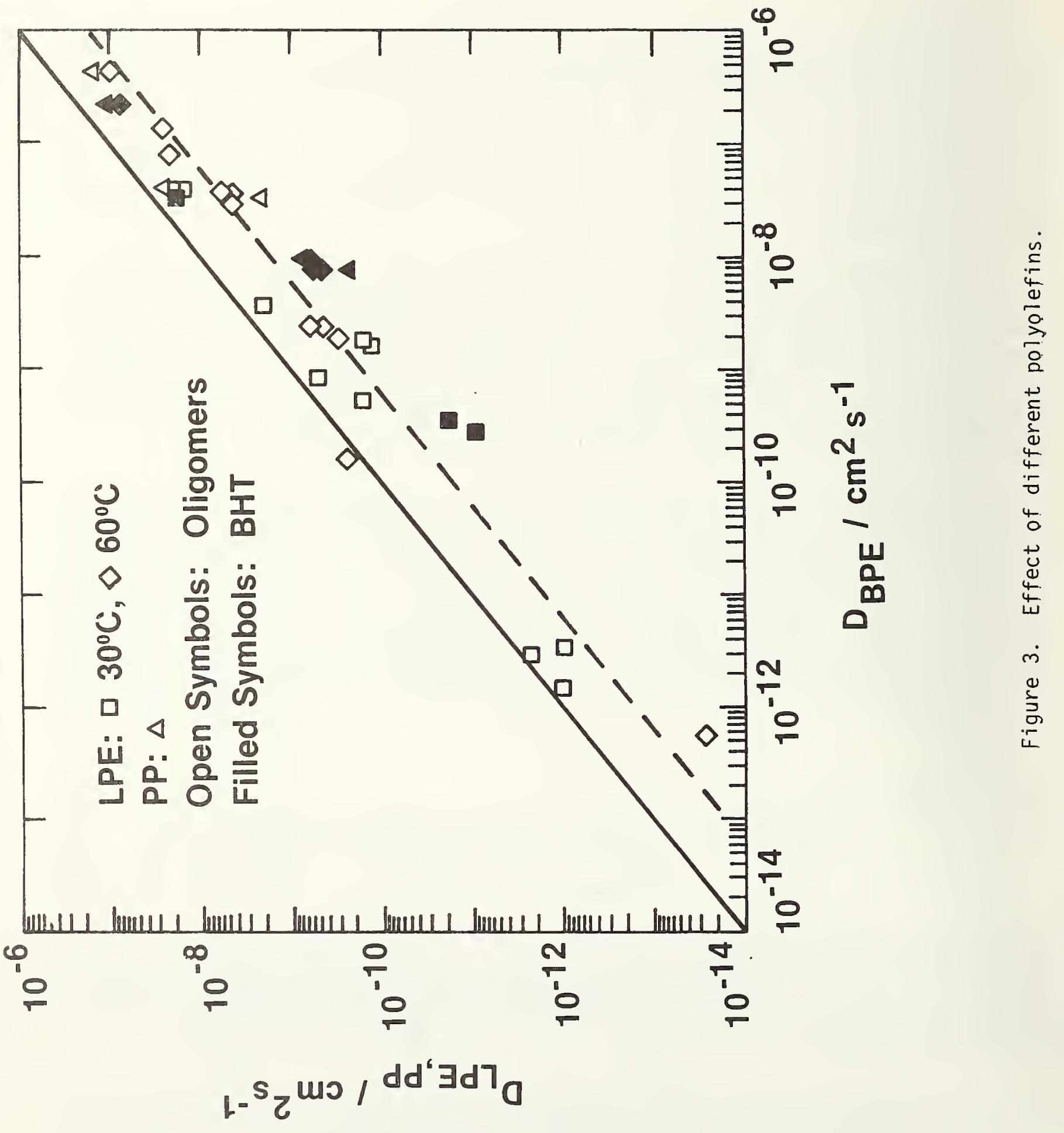


References:

[1] P. G. de Gennes, Macromolecules, 9, 594 (1976).

[2] P. G. de Gennes, J. Chem. Phys. 55, 572 (1971).

[3] H. Hervet, L. Léyer, and F. Rondelez, Phys. Rev. Lett. 42, 1681 (1979).

[4] D. E. Koppe1, D. Axelrod, J. Schlessinger, E. L. E1son, and W. W. Webb, Biophys. J. 16, 1315 (1976).

\section{Assessment of Transport Mechanisms For Radon In and Through Building Materials}

\section{R. J. Rubin}

Accumulation of radioactive radon gas in buildings poses a health hazard. High levels of radon are encountered in certain geological areas where the naturally occurring amounts of radium-226 are high. Certain building materials, such as concrete, building blocks, and gypsum board, may also produce higher than average amounts of radon if one of the components of the building material contains high trace concentrations of radium-226. Under an interagency agreement with the EPA, NBS has very recently undertaken the task of studying radon transport in building materials. The study involves a cooperative effort between three NBS Centers: Radiation Research, Building Technology, and Materials Science. The initial phase of this project, to critically assess the current state of knowledge of radon transport through or emanation from building materials, has been completed. Because of our expertise in diffusion and transport models, the Polymer Science and Standards Division was asked to assess current mechanisms and models and apply them to some selected cases.

Models of the movement of radon through and from porous building materials such as concrete are based on the assumption that the transport of radon through the air-filled pore system of a porous material occurs in response to two driving forces: (1) diffusive transport of radon in response to a difference in radon concentration, where the transport coefficient is the diffusion coefficient of radon in the air-filled pore system, and (2) convective transport of an air-radon mixture in response to an air pressure difference, where the transport coefficient is the permeability coefficient of air in the pore system. Among the questions and transport cases which have been treated are: (1) What are the relative contributions of concentration differences and pressure differences to the steadystate transport of radon in concrete? From literature values of the diffusion coefficient of radon in concrete, the porosity of concrete, and the permeability coefficient of air in concrete, we have found that a pressure difference of $25 \mathrm{~mm} \mathrm{Hg}$ across $10 \mathrm{~cm}$ of concrete significantly enhances the flux of radon through a surface. (2) Estimates are obtained of the times required to establish steady-state behavior in both the case of diffusive transport and convective transport. In general, the time required for establishing steady-state convective transport is a few hours when the time required for establishing steady-state diffusive transport is a few days. Thus, it appears that short term variations in radon concentration (of the order of one day or less) are "averaged out." (3) The factor by which the steady-state radon flux from bare soil is reduced when a concrete slab is interposed has been calculated.

These and other examples are incorporated in the report, "Review and Assessment of Radon Transport Through and From Building Materials," which has been reviewed favorably by a number of experts in the field and will be published as an NBS Technical Note. Among the recommendations which have been made on the basis of our study for this report are: (1) The time-dependent transport equations for transport of radon in a porous medium which include both diffusive and convective terms should be verified or validated. (2) Measurements of the diffusion coefficient of radon and the permeability coefficient of air should be made on the same concrete specimens. It is only through such combined measurements that the relative magnitude or importance of the two kinds of transport can be determined with certainty. (3) An experiment has been proposed to determine the diffusion coefficient of radon in concrete from measurements of the build-up of radon concentration with time when a specimen of welldefined shape is placed in a radon chamber. The model case of a cylinder with exposed ends and coated side has been analyzed. 


\section{Task Leader}

Polymeric materials increasingly are used in applications where long-term mechanical durability is critically important. The full exploitation of these materials would result in greater productivity through improved properties at lower costs. The realization of increased productivity is often limited by the lack of data and models upon which to design for long-term use. Thus, the lack of adequate test methods and data for evaluation of long-term mechanical performance not only restricts applications, but also leads to over design which results in more expensive items and limits weight savings. The substitution of polymer composites for critical materials in the aerospace industry has been limited for this reason. Evaluation and control of materials parameters that affect long-term performance should lead to more durable materials and, hence, reliable performance for the intended service life.

The objective of this task is to provide data, test methods, and standards for evaluation of long-term mechanical durability of polymeric materials. The approach is to identify and analyze principal failure mechanisms in these materials under mechanical stress in the use environment, to determine the effects of chemical structure and fine structure morphology on mechanical properties and failure processes and to develop constitutive relationships and models for mechanical properties as the basis for accelerated test methodology and lifetime prediction.

New activities have been initiated in glassy and composite polymeric materials. Theories have been developed to predict the rate of craze extension and stress relaxation at crack tips in crazes. The fracture energy of elastomer-modified epoxy resin systems has been investigated to elucidate mechanisms of crack blunting in these composite materials. The identification of failure modes both at the fine structure and molecular level not only serves as a basis for models to predict long-term behavior but also leads to quality control methods during processing. For example, incomplete chemical reactions during curing of composite materials have been shown to affect long-term mechanical performance. The extent of these chemical reactions during the cure process may be determined using spectroscopic techniques of nuclear magnetic resonance and Fourier transform infrared spectroscopy.

The National Bureau of Standards has established joint programs on mechanical durability with other Federal agencies which are fostering the application of polymeric materials in areas of national concern. A joint program with the Department of Energy is concerned with the mechanical performance of elastomeric seals in geothermal energy wells. The Bureau of Medical Devices of the Food and Drug Administration supports an investigation of relationships between the fine structure morphology of ultra high molecular weight polyethylene and the long-term mechanical properties of this material when used in medical implant devices. Improving the mechanical durability of fibrous network materials is the goal of a joint program with the Bureau of Engraving and Printing. In all of the above applications, there exists the need for test methods and standards to ascertain the physical limits of polymeric materials in various use environments. An outgrowth of a recent project with the Department of Transportation on mechanical durability of plastic shipping containers has been the development of a new test for environmental stress cracking. Optimal testing conditions are being identified for submission to the ASTM of an improved standard method for environmental stress-crack resistance.

\section{Molecular Processes Associated with Mechanical Deformation and Failure}

B. M. Fanconi, D. L. VanderHart, S. L. Wunder, J. P. Colson, S. J. Kryder, E. E. Toth, K. L. DeVries ${ }^{1}$, S. Abbate ${ }^{2}$, and G. Zerbi ${ }^{2}$

The elucidation of molecular processes and structure changes accompanying mechanical deformation and failure not only provides the basis for development of test methods for long-term performance and quality control but also provides insight into the selection and design of materials with improved mechanical performance and durability. The semicrystalline structures found in most polymeric materials present significant challenges to characterization of molecular structure. New spectroscopic techniques involving ${ }^{13} \mathrm{C}$ nuclear magnetic resonance and Raman scattering are developed to elucidate molecular conformations in disordered material. Reorganization of polymer molecules during deformation affects mechanical performance. Identification of the molecular processes involved and the physical structures which result are the aim of the NMR studies.

Fourier transform infrared spectroscopy is used to determine the number of chain scissions produced per free radical measured during fracture in order to assess the role of free radical chain propagation reactions in the fracture process.

\footnotetext{
Tniversity of Utah

${ }^{2}$ Instituto di Chimica Industriale di Politechnico
} 
Spectroscopic Investigations of Mechanical Degradation of Polyethylene.

Molecular processes associated with mechanical degradation of polymeric materials include chain rupture or scission. It has been postulated that chain rupture sites may be highly localized forming microvoids from which cracks may initiate leading to macroscopic failure [1]. The process of bond scission produces free radicals that may initiate many additional chain scissions before recombination to form stable chemical species. This would suggest that mechanical stability might be improved by the addition of free radical scavengers. The evidence for multiple chain scissions per free radical comes from comparisons of the number of free radicals generated during mechanical degradation measured by electron spin resonance and new end groups formed as determined by infrared spectroscopy [1]. 0ther estimates of the extent of chain rupture derive from viscosity-averaged molecular weight changes [2]. The number of chain scissions per free radical determined by viscosity measurements on mechanically damaged polyethylene is several orders of magnitude lower than the ratio evaluated from previous infrared data.

Previous infrared determinations were based on spectral differences between deformed and undeformed specimens [1,3]. Effects of molecular orientation, changes in sample thickness and surface texture, as well as differing void content could not be readily accounted for in these measurements. Another complication derives from the use of specimens with relatively high initial concentrations of end groups. For these reasons, we have carried out a detailed investigation of free radicals and chain scissions during mechanical degradation of polyethylene using electron spin resonance and Fourier transform infrared spectroscopy.

Measurements were made on tensile-fractured polyethylene fibers, rods ground in liquid nitrogen, and on an ultra-high molecular weight polyethylene that was also mechanically degraded through grinding in liquid nitrogen. This last sample was of particular interest in that the undeformed material contained very few end groups. To circumvent spectral effects due to orientation, surface conditions, void content, and film thickness caused by the deformation, our approach was to cast a film of the deformed material from solution or the melt. Films for IR analysis were prepared from the reference material in an identical manner.

Spectral changes from new end groups were observed in the 900 to $1000 \mathrm{~cm}^{-1}$ region (arising from vinyl groups), near $1375 \mathrm{~cm}^{-1}$ (due to methyl groups), and in the 1700 to $1750 \mathrm{~cm}^{-1}$ region (attributed to carbonyl groups). Increases were observed in the concentrations of the terminal vinyl, methyl, and aldehyde groups. The concentrations of new end groups are given in table 1 and compared to the free radical concentrations in table 2 .

As evident from table 2, we find that the ratio of chain scissions to free radicals is lower by one to two orders of magnitude when compared to previous determinations [1]. 0ur values are still approximately an order of magnitude larger than the viscosity-determined values [2]. We attribute the latter discrepancy to differences in molecular weight and the physical state of the samples used in the two studies. The discrepancy with previous IR studies [1,3] arises from sampling methods and the errors in the previous work associated with taking differences between relatively intense bands. In the case of the ultra high molecular weight polyethylene, no change was observed in the vinyl group concentration, even though the initial concentration of this species was extremely low.

Examination of Drawn PET Fibers by ${ }^{13} \mathrm{C}$ NMR. Through a collaborative effort with the Firestone Tire and Rubber Company, several drawn PET fibers having different annealing histories at various constraints were investigated. Solid state ${ }^{13} \mathrm{C}$ NMR measurements were made at NBS on specimens furnished by Firestone and characterized by them with respect to mechanical properties, $x$-ray scattering, and density. The motivation behind this research was to determine whether solid-state ${ }^{13} \mathrm{C}$ NMR could: (1) clarify the concept and/or measurement of crystallinity in these fibers, (2) determine noncrystalline orientation, and (3) aid in relating molecular properties to mechanical properties.

Limited variable temperature data were obtained on non-spinning oriented samples. The rotation of a large number of aromatic rings about the 1,4 axis at frequencies near one kilohertz was observed in the temperature range of 130 to $150^{\circ} \mathrm{C}$. At room temperature, a few mobile aromatic rings were observed to rotate at similar frequencies. In these experiments, ring rotation is observed preferentially for those chains which have been oriented by drawing and/or annealing. The question of the morphological origin of these chains remains somewhat ambiguous since there has been no clear definition of crystallinity in the ${ }^{13} \mathrm{C}$ NMR results to date. Possible definitions of crystallinity in terms of lineshapes (spinning or non-spinning) or relaxation ( $T_{1 C}$ or $T_{1 \rho}^{C}$ ) have all given ambiguous results. K-ray results suggest that the crystallite block length is rather short ( 3.1 to $8.0 \mathrm{~nm})$ and that percent crystallinities are low (20 to 40 percent). On the other hand, NMR results, particularly spinning lineshape analysis, suggest percent crystallinities in the range of 60 to 70 percent. We are considering using Raman or IR in future work on these samples to measure the gauche/trans ratio and how this ratio is related to the NMR and $x$-ray observables.

The methylene resonance in these non-spinning oriented samples is rather distinct from the rest of the ${ }^{13} \mathrm{C}$ NMR resonances. From this resonance, overall orientation can be determined. This analysis has not, as yet, been done in detail, but qualitatively, the following statements can be made based on the observed NMR lineshapes: 
Table 1

Concentrations of Chemical Groups Formed During Mechanical Degradation of Polyethylene

\begin{tabular}{|c|c|c|c|c|c|}
\hline Group & $\begin{array}{l}\text { IR Band Freq. } \\
\underline{\left(\mathrm{cm}^{1}\right)}\end{array}$ & Commercial & $\begin{array}{c}\text { Concentrations } \\
\text { (No./cc) }\end{array}$ & Fractured PE & Ref. 1 \\
\hline $\mathrm{RCH}=\mathrm{CH}_{2}$ & 909 & $1.2 \pm 0.4 \times 10^{17}$ & - & $1.3 \pm 0.4 \times 10^{17}$ & $3.4 \times 10^{18}$ \\
\hline $\mathrm{RCH}=\mathrm{CHR}^{\prime}$ & 965 & - & - & - & $1.9 \times 10^{18}$ \\
\hline $\mathrm{RCH}_{3}$ & 1,370 & $2.2 \pm 1 \times 10^{18}$ & $5.6 \pm 3 \times 10^{18}$ & $7.3 \pm 4 \times 10^{17}$ & $11.8 \times 10^{18}$ \\
\hline $\mathrm{RCHO}$ & 1,735 & $3.7 \pm 0.5 \times 10^{17}$ & $1.1 \pm 0.1 \times 10^{18}$ & $1.9 \pm 0.6 \times 10^{17}$ & $4.6 \times 10^{18}$ \\
\hline
\end{tabular}

Table 2

Chain Scissions Per Free Radical

Ground PE

Commercial
$1+1 \times 10^{17}$

$1.2 \times 10^{16}$

$34-50$ $\underline{\text { UHMW }}$

$1.2 \pm 0.1 \times 10^{18}$

$1 \times 10^{17}$

$11-13$
Tensile Fractured PE

Ref. 1
No. chain scissions per gram

No. free radicals per gram

Chain scissions per radical

$\begin{array}{ll}5.1 \pm 1 \times 10^{17} & 1.2 \pm 0.1 \times 10^{18} \\ 1.2 \times 10^{16} & 1 \times 10^{17} \\ 34-50 & 11-13\end{array}$

$3.4 \pm 1 \times 10^{17}$

$9.9 \times 10^{18}$

$3.5 \times 10^{15}$

$5 \times 10^{15}$

$68-125$

1,600

(1) Annealing at constant length improves the orientation of those chains which were already quite well aligned by the cold drawing. However, there is no appreciable change in the amount of new orientation of those chains whose direction deviated substantially from the draw direction.

(2) Annealing with free ends broadens the orientational distribution for the highly-oriented chains and significantly increases the number of poorly-oriented segments.

Examination by NMR of Morphological Changes in the Wet Beating of Cellulosic Materials. A collaborative effort with the Bureau of Engraving and Printing entails the examination of paper-related cellulosic materials. The background for this work was that in previous studies [1] it was shown that NMR could distinguish ${ }^{13} \mathrm{C}$ resonances associated with cellulose chains near the surface as opposed to the interior of an elementary fibril. Elementary fibrils are roughly $3.5 \mathrm{~nm}$ in diameter.

In paper making, cellulosic materials are soaked in water and then wet beaten. The latter process seems to plasticize the materials so that they form a good paper. An important question concerns the fate of the elementary fibril during wet beating. It is known, for example, that wet beating cotton increases the amount of non-freezing water, while the fiber saturation point increases in wood pulp as a result of this process. So, in general, the amount of water intimately associated with the cellulose increases with wet beating.

For this project, a high speed water-tight rotor had to be designed which could spin stably at frequencies of at least $1.5 \mathrm{KHz}$. Such a rotor was designed, and data were obtained using dry, wet, and wet beaten samples of microcrystalline cellulose, Norway Spruce Kraft pulp, and purified cotton. The NMR results showed changes in the surface-carbon resonances on going from dry to wet samples. But no further changes were observed when going to the wet beaten material. Thus, it appears that wet beating does not break up the elementary fibrils to any significant (> 10 percent) degree. Some questions remain as to whether all of the carbons are being observed, but this problem cannot be addressed unless we acquire the ability to observe proton resonances directly. 
Study of Crystal Modification and Surface Area Changes in Mechanically-Deformed Ultra High Molecular Weight Linear Polyethylene. In FY80, we observed that cold (room temperature) drawn $(\lambda=2.7)$ ultra high molecular weight linear polyethylene contained a significant portion of the monoclinic crystalline phase along with the usual orthorhombic phase. This year, the amount of this phase was quantified using magic angle spinning (MAS) ${ }^{13} \mathrm{C}$ NMR. Furthermore, by taking advantage of magnetization diffusion of proton magnetization in solids along with the cross-polarization process between ${ }^{13} \mathrm{C}$ and ${ }^{1} \mathrm{H}$ nuclei, an attempt was made to measure the increase in going from the undrawn to the drawn LPE. The results, simply interpreted, indicated a significant increase (> $2 x$ ) in surface area in the drawn material. However, unambiguous interpretation awaits the ability to observe proton resonances directly.

Spectroscopic Investigation of Disorder in Polyethylene. Characterizastion of order at the molecular level is fundamental to establishing connections between molecular structure and mechanical properties. The structure of crystalline material can often be deduced from x-ray scattering data, however, this is not the case for molecular conformations in disordered material. In concert with suitable calculations of frequencies and intensities, vibrational spectroscopy can provide useful information on disordered polymeric materials. A case in point is the $\mathrm{C}-\mathrm{H}$ stretching region of polymethylenes which is sensitive to both intra- and intermolecular order. In the Raman spectra of these molecules, there is a Fermi resonance interaction between the $\mathrm{C}-\mathrm{H}$ bending and stretching vibrations, which depends on the state of the polymethylene chain. In the approximation that a gauche bond can be modeled by a decoupled $\mathrm{CH}_{2}$ group, the Fermi resonance scheme for this system can be compared to that for the al]-trans chain. The calculations indicate that a redistribution of intensity in the $\mathrm{C}-\mathrm{H}$ stretching region occurs upon introduction or disordered structures in the all-trans chain. In particular, a band at $2935 \mathrm{~cm}^{-1}$ increases relative to a band at $2850 \mathrm{~cm}^{-1}$ as the population of gauche bonds increases. This was demonstrated to occur for semicrystalline polyethylene of varying percent crystallinities and for melt polyethylene as a function of temperature.

\section{References:}

[1] S. N. Zhurkov, V. A. Zakrevskii, V. E. Korsukov, and V. S. Kuksenko, Sov. Phys. Solid State, 13, $1680(1973)$.

[2] T. M. Stoeckel, J. Blasius, and B. Crist, J. Polym. Sci., Polym. Phys. Ed., 16, 485 (1978).

[3] D. L. Tabb, Ph.D. Thesis, 1974, Case Western Reserve University.

[4] D. L. VanderHart and W. L. Ear], J. Am. Chem. Soc., 102, 3251 (1980).

Relationship Between Morphology and Mechanical Properties of Polyethylene.

G. B. McKenna, F. A. Khoury, J. M. Crissman, L. H. Bolz, G. W. Bullman, and G. M. Martin

The mechanical properties of semicrystalline polymers and their durability are related to the fine structure morphology. The work of this subtask is to determine these relationships for polyethylene, a widely used semicrystalline polymer. The morphology is determined by processing conditions and, to a lesser extent, by the use environment. An examination of processing conditions on morphology is an important aspect of the work. To improve our knowledge of the organization of polymer chains in bulk crystallized polyethylenes, studies have been carried out on solution crystallization at high temperatures. These conditions produce morphological features similar to those found in melt crystallized material with the advantage that detailed morphological examinations using electron microscopy may be made without subjecting the samples to harsh chemical treatments.

The Bureau of Medical Devices of the FDA supports investigations into the relationships between morphology and mechanical performance and durability of ultra high molecular weight polyethylene.

Relationship Between Morphology and Mechanical Properties of Ultra High Molecular Weight Polyethylene. Uitra high molecular weight polyethylene (UHMWPE) is used in a variety of orthopedic joint prostheses. One factor which limits the expected lifetime of a prosthesis is the durability of the UHMWPE component. In clinical use, failure of the polymer component is not a result of wear alone but may occur through creep, fatigue associated with wear, or even fracture. The length of time between device implantation and clinical failure depends upon the time dependent mechanical response of UHMWPE to the clinical loading environment.

It is known that the properties of semicrystalline polymers are highly affected by such structure or morphology related features as degree of crystallinity, orientation, and spherulite size and texture. These features are determined by processing variables such as temperatures and pressures used during fabrication of a polymer component. Under contract with the Food and Drug Administration's Bureau of Medical Devices, we are studying the relationship between morphology and the mechanical behavior of UHMWPE. An important consideration in this work is the determination of the material behavior variability which can be introduced by changes in the processing conditions and molecular weight so long as the molecular weight remains in what is considered to be the UHMWPE range, i.e., $M_{W}>10^{6}$. 
Protocols have been established for preparing compression molded sheets and solid cylinders of the UHMWPE. Features of the fine structure of both the raw polymer and the compression molded sheets and cylinders have been examined. Three aspects of the raw polymer have been studied: its morphological characteristics, its melting behavior, and its crystallinity. The polymer, as received, is in its as-polymerized state and consists of fine particles. Because of its very high melt viscosity, both molded and machined prosthesis components made from it retain a memory of the particulate nature of the raw polymer. This was also true for specimens molded in our laboratory at temperatures as high as $90 \mathrm{~K}$ above the melting point. This observation emphasizes the importance of characterizing the structure of UHMWPE products, not only to determine their crystallinity but also to monitor those aspects of their morphology which are directly related to, and a consequence of, the particulate nature of the raw polymer. Since the nature of the shapes, sizes, and fine textures of nascent polyethylene particles vary depending upon the polymerization conditions, knowledge of the morphology of the raw polymer is a necessary aspect of the present study.

Differential scanning calorimetry (DSC) was used to examine the melting behavior and crystallinity of the raw polymer. The melting point was found to be between 413 and $414 \mathrm{~K}\left(140\right.$ to $141{ }^{\circ} \mathrm{C}$ ). The percent crystallinity, as determined from the area under the melting curve, was 78 percent, which represents a value significantly higher than can be expected for the same material compression molded at temperatures above the melting point.

The mechanical behavior of the processed polymer has been examined for a variety of different deformation histories including single step stress relaxation, uniaxial creep, constant rate of strain, and cyclic loading. Stress relaxation and creep experiments have been done for material which was quenched from the melt (density $=0.923 \mathrm{~g} / \mathrm{cm}^{3}$ ), slowly cooled from the melt $\left(0.935 \mathrm{~g} / \mathrm{cm}^{3}\right.$ ), and slowly cooled followed by annealing at a temperature near the melting point $\left(0.942 \mathrm{~g} / \mathrm{cm}^{3}\right)$. Quenching of the polymer reduced the modulus at small strains by about a factor of two from that of the slowly cooled material, whereas annealing increased the modulus only by about 20 percent. 0ne interesting feature of this material is that unlike lower molecular weight polyethylenes, in the quenched or slowly cooled state it does not exhibit the phenomenon of necking during a creep or constant rate of elongation experiment. However, upon annealing necking does occur.

The uniaxial creep behavior of the quenched and slowly cooled materials has been mapped for stresses in the range from nine to $27.5 \mathrm{MPa}$ and time of from $10^{2}$ seconds to $10^{7}$ seconds ( $\sim$ one/year). Although the quenched material is more compliant than the slowly cooled UHMWPE, the maximum stretch attainable was essentially the same for both $(\lambda \sim 6.0)$. This is significantly less than is achievable in lower molecular weight polyethylenes which neck and then cold draw. The creep response of the annealed material was found to be very nearly the same as that of the slowly cooled polymer.

The cyclic fatigue behavior has been studied for two different UHMWPE samples which, based upon intrinsic viscosity measurements, have molecular weights of $M_{W} \sim 4.5 \times 10^{6}$ and $2.5 \times 10^{6}$. Tests were carried out at different levels of stress and frequency, and it was found that the fatigue lifetimes of both samples decreased with increasing test frequency. This behavior is the opposite of that for lower molecular weight polyethylenes $\left(M_{W}<3 \times 10^{5}\right)$. Further, it was found that the lifetime of the material having the lower molecular weight was about 30 percent higher than that having the higher molecular weight. This behavior is also unlike that of the much lower molecular weight polyethylenes where the fatigue lifetime generally increases with increasing molecular weight. Sorting out the respective influences of molecular weight and density (fine structure) will require a comparison of both high and low molecular weight polymers having similar densities.

We have also begun to characterize the environmental stress crack resistance (ESCR) of the UHMWPE polymer prepared under varied processing conditions. The experimental technique being used to evaluate the ESCR is the same as that recently developed by us for application to other ethylene-based plastics. Preliminary results indicate that the ESCR is greatest for the slowly cooled material and least for the annealed UHMWPE.

The Morphology of Compression Molded Ultra High Molecular Weight Polyethylene (UHMWPE). The work described below is part of a continuing project sponsored by the FDA and the objectives of which are to characterize the mechanical properties of UHMWPE and to examine the relationships between these properties and the morphology of the polymer. These relationships are of particular interest to the FDA because numerous components of artificial orthopedic implants (e.g., hip cups and tibial plateaux) are made from this polymer.

We have previously reported that sheets of UHMWPE (Mol. Wt. $\sim 4 \times 10^{6}$ ) which were compression molded at $200{ }^{\circ} \mathrm{C}$ and then slowly cooled to room temperature did not exhibit any necking when stretched uniaxially at a strain rate of $0.02 / \mathrm{min}$ up to the breaking strain. The density of the undeformed sheets prior to deformation was $0.936 \mathrm{~g} / \mathrm{cm}^{3}$. The absence of necking contrasts with the behavior of lower molecular weight linear polyethylene. More recently, the recovery of samples from uniaxial deformation to various strains below the breaking strain has been examined. The sheets exhibited considerable recovery. For example, sheets were stretched at $37{ }^{\circ} \mathrm{C}$ and at a strain rate of $0.02 / \mathrm{min}$ to strains of $0.25,0.5,1.0,2.0$, and 3.0 , respectively. They were then released and allowed to cool 
to room temperature under ambient conditions. The corresponding residual strains after 24 hours at room temperature were $0.06,0.12,0.34,0.92$, and 1.15 .

Wide angle and small angle x-ray diffraction patterns were obtained from the samples originally stretched to strains of $1.0,2.0$, and 3.0 at $37{ }^{\circ} \mathrm{C}$. The $x$-ray beam was normal to the stretching direction. In the case of the wide angle patterns, the changes associated with increasing residual strain in the released samples were characterized by a progressive intensification of the 200 arcs in the equatorial region. Correspondingly, the 110 arcs, which were split about the equator, tended to be closer to the equatorial direction with increasing residual strain. In addition to the reflections associated with the usual orthorhombic unit cell of polyethylene, all three samples exhibited fainter reflections characteristic of a metastable polymorphic form of polyethylene, which form has been variously described as being monoclinic or triclinic [1]. The main feature exhibited by the small angle diffraction patterns from the same three samples was that as the residual strain increased, the patterns became more clearly "four-pointed" in character. This latter feature indicates the occurrence, between crystalline and disordered regions, of interfaces which are appreciably inclined relative to the stretching direction. In addition, the samples originally stretched to strains of 2.0 and 3.0 exhibited a central horizontal streak indicating the presence of long and comparatively narrow voids whose long dimension is oriented parallel to the stretching direction.

Examination of the samples mentioned in the previous paragraph in a scanning electron microscope indicated that although they did not exhibit any macroscopic necking when originally strained, stretching did not occur uniformly throughout each sample. The manifestation of nonuniform deformation was evidenced by the occurrence of regions which exhibit an apparent "fibrillar" appearance. These regions of higher deformation, in which the "fibrils" were oriented parallel to the stretching direction, were interspersed between regions of lower deformation which exhibited no evident fibrillar character. It remains to be determined whether this nonuniform deformation is associated with the memory, which the molded samples retain, of the grain-like character of the constituent particles of the raw UHMWE polymer. This matter is currently under investigation.

The Habits of Polyethylene Crystals Grown From Solution at High Temperatures. The two main objectives of this investigation have been the following. First, to seek appropriately "poor" solvents from which chain folded polyethylene crystals could be grown which exhibit $\{110\}$ and $\{200\} 1$ lateral faceting and preferential growth parallel to the b-axis (i.e., axial ratios B/A $>1.33^{*}$ ) at temperatures which approach and overlap the temperature range ( 118 to $130^{\circ} \mathrm{C}$ ) in which the polymer crystallizes isothermally in the form of axialites and spherulites upon cooling from the molten state. Second, to characterize the lateral growth habits and conformations (non-planar character, e.g., hollow pyramidal or variously curved) of crystals having B/A > 1.33. Interest in the morphology of such crystals and its dependence on molecular weight and crystallization temperature stems from their potential usefulness as models for the experimentally less accessible constituent lamellae in the various axialitic and spherulitic structures which are formed in bulk (melt) crystallized polymer [2]. It has long been known that the preferred growth direction in the radiating lamellae in polyethylene spherulites is parallel to their $\underline{b}$-axis. Notwithstanding the new insights provided by 011 ey, Hodge, and Bassett [3], the origins of the twisting of the lamellae in the spherulites as well as other aspects of the morphology of these lamellae remain obscure.

Using dodecanol as a solvent and two polyethylene fractions, Fraction $I(M n=11400)$ and Fraction II $(M n=28900)$, the highest temperatures at which crystallization has been achieved to date from 0.01 percent solutions have been $115{ }^{\circ} \mathrm{C}$ (Fraction I) and $120^{\circ} \mathrm{C}$ (Fraction II), at which temperatures crystals having an axial ratio $B / A \sim 6.0$ were formed. Examination of crystals of both fractions grown at $105^{\circ} \mathrm{C}$ and above revealed that in addition to the fact that in both cases higher crystallization temperatures yielded crystals with a higher axial ratio, for given crystallization temperatures in the range 105 to $115^{\circ} \mathrm{C}$, crystals of the higher molecular weight fraction exhibited a smaller axial ratio. For example, the axial ratios of crystals of Fractions I and II grown at $115{ }^{\circ} \mathrm{C}$ were $\sim 6.0$ and $\sim 2.4$, respectively. This effect indicates that the axial ratio and, hence, ratio of the growth rates perpendicular to the $\{110\}$ and $\{200\}$ planes are governed primarily by the undercooling $\Delta T$.

Various "self-seeding" procedures are being explored in an attempt to grow crystals of the two fractions at even higher temperatures. In addition, the effect of crystallization temperature on the habits of crystals of a third polyethylene fraction (Fraction III, Mn = 100500) is being pursued using the same solvent. Concurrently, experiments aimed at deducing the nature of the as-grown conformations of crystals exhibiting axial ratios in the range 1.7 to 6.0 are continuing. Scanning transmission electron microscopy and small area electron diffraction are the main tools being used in this connection. Among the difficulties encountered in characterizing the conformation of the crystals are the following features which have been encountered in the few species of crystals which have been explored so far: (a) variations in the tilt of the chain stems relative to the crystal surfaces both along and across the length of the crystals, and (b) apparent differences in stem tilts between crystals of different sizes grown at the same temperature and of the same polymer fraction. The latter effect, which has been encountered during the course of a comparison between crystals of Fraction $\mathrm{I}$ grown at $115{ }^{\circ} \mathrm{C}$

Where $B$ and $A$ are the dimensions of the crystals parallel to [010] and [100], respectively. 
from unseeded preparations (large crystals) and "self-seeded" preparations (smaller crystals), is currently under study.

References:

[1] See, for example, A. Turner Jones, J. Polym. Sci. 62, 553 (1962).

[2] F. A. Khoury, Faraday Disc. Chem. Soc. 68, 404 (1979).

[3] J. Polym. Sci., Polym. Phys. Ed. 17, 627 (1979).

\section{Fracture Processes in Polymers}

\section{E. Passaglia, D. L. Hunston, and J. L. Rushford}

At the macroscopic level, the onset of failure under mechanical stress can be identified with either inhomogeneous deformation (necking) or crack growth. Two aspects of crack growth that are particular to polymers are craze phenomena and time dependent fracture. Glassy polymers exhibit craze formation in the region preceding the crack tip. The failure of these materials is related to the growth of the craze region and its dependence on stress relaxation processes of the material. An adequate theoretical description of craze growth and its dependence on material properties is the aim of a recently initiated effort in the fracture of polymers. A successful model of the craze phenomena would provide an improved basis for lifetime prediction.

A second activity in a study of the fracture of polymers is concerned with elucidating the mechanisms by which elastomer additives improve the fracture toughness of epoxy matrix composites and the dependence of these mechanisms on time and temperature. Test methods are being developed that adequately measure the resistance of glass fiber reinforced composites to delamination. The dependence of the failure time of elastomer modified composites on temperature has been established. An Arrheniustype temperature dependence is found that may be the basis for an accelerated test method.

Theoretical investigations of craze and crack growth. The investigation of the effect of relaxation of stresses within a craze at a crack tip on the rate of craze and crack growth is continuing. $A$ manuscript entitled, "Relaxation of Stresses in Crazes at Crack Tips," has been completed and is undergoing editorial review.

For this investigation, the theory of cohesive stresses of Barenblatt [1] was applied to the craze growth problem, considering the uncrazed material to be elastic. In the Barenblatt theory, the magnitude $\sigma$ of the cohesive (craze) stresses and the distance $\alpha$ (craze region) over which they act are given (for very small craze regions) by the equation

$$
\int_{0}^{\alpha} \frac{\sigma(\xi, t)}{\sqrt{\xi}} d \xi=\sqrt{\pi / 2} k_{1}
$$

where $\xi$ is a coordinate within the craze and $K_{1}$ is the applied stress intensity factor. This is related to the applied stress $\sigma_{\infty}$ and the crack length a by the equation

$$
K_{1}=\sigma_{\infty} \sqrt{\pi a} f(a / w)
$$

where $w$ is the specimen width and $f(a / w)$ is a geometric factor determined by the specimen configuration.

The fundamental hypothesis of the Barenblatt theory is that the cohesive (craze) stresses cancel the stress singularity that would exist at the crack tip in their absence. In the present treatment, it is assumed that this singularity cancellation exists even for moving crazes. It is then clear from Eq. (1) that, for constant $K_{1}, \alpha$ must increase if $\sigma$ decreases. A simple equation relating the rate of craze growth to rate of stress relaxation is obtained from differentiation of Eq. (1):

$$
\frac{\sigma(\alpha, t)}{\sqrt{\alpha}} \frac{d \alpha}{d t}=\sqrt{\pi / 2} \frac{d K_{1}}{d t} \int_{0}^{\alpha} \frac{\partial \sigma}{\partial t} \frac{d \xi}{\sqrt{\xi}}
$$

where $\sigma(\alpha, t)$, is the stress at the trailing edge of the craze (i.e., the tip of the crack).

This integro-differential equation is the basic equation for this type of craze growth, called "relaxation controlled growth" by Williams and Marshall [2]. Its solution requires knowledge of the rate of change of craze stress over the whole craze region. This latter problem can be formulated in terms of integral equations, but these are in general intractable. However, it can be said that the 
rate of relaxation of stress within the craze will in general be lower than the rate of the relaxation of stress for craze material in a normal (i.e., fixed length) stress relaxation experiment.

With some simplifying assumptions, Eq. (3) may be solved. The assumptions are:

(1) The craze initiation stress at the craze tip is a constant $\sigma_{0}$. This is equivalent to a stress criterion for craze initiation, which is considered reasonable for the triaxial stress state at the craze tip.

(2) At $t=0$, i.e., at the time of initial craze formation, the stress in the craze is independent of position within the craze, as in the Dugdale model [3]. This stress then relaxes with time, except at the craze tip, where growth occurs. This is called the "generalized Dugdale model." yield

(3) The stress intensity factor is a constant. Under these conditions, Eq. (3) may be solved to

$$
\sqrt{\alpha} \sqrt{t}=\frac{1}{4} \sqrt{\frac{\pi}{2}} k_{1} \int_{0}^{t} \frac{d t}{\sqrt{t} \sigma(\alpha, t)}
$$

Assuming now that $\sigma(\alpha, t)$ varies exponentially with time as follows

$$
\sigma(\alpha, t)=\frac{\sigma_{0}}{1+(t / \tau)^{m}}
$$

where $r$ is in the nature of a relaxation time, and $m$ is related to the slope of the compliance on a log- $\log$ plot, Eq. (4) may be integrated to give

$$
\alpha=\frac{\pi}{8} \frac{k_{1}^{2}}{\sigma_{0}^{2}}\left[1+(t / \tau)^{m} \frac{1}{2 m+1}\right]^{2}
$$

For times long compared to $\tau$, this equation gives satisfactory agreement with experimental results and gives the proper dependence on $K_{1}$. For short times, the relevant experiments are complicated by the presence of solvents. Clearly, the exact form of the solution will depend upon the time dependence taken for $\sigma(\alpha, t)$ in Eq. (4).

Present work is concerned with investigating more thoroughly the equations that determine the time behavior of the stress within the craze.

Experimental investigation of craze growth. Considering the importance to craze growth of the relaxation of stresses within the craze, a small experimental program to measure the creep function in craze material was begun. For this purpose, a sample of polycarbonate with large crazes grown in it was kindly provided by Dr. Roger Kambour of the G.E. Research Laboratory. This was then mounted on a jig, loaded to a stress of $800 \mathrm{psi}\left(0.56 \mathrm{mN} / \mathrm{m}^{2}\right)$, and the thickness of the craze measured microscopically for a period of 24 hours. While the experiment suffered from lack of precision, preliminary results indicate that the function

$$
\frac{J(t)-J(0)}{J(0)}
$$

where $J(0)$ is the instantaneous creep compliance and $J(t)$ is the creep compliance at time $t$, is the same for the craze material as for the uncrazed substrate. This implies that the retardation times for creep are unchanged by the process of crazing, but this result will have to be confirmed in more careful experiments.

Influence of Matrix Properties on Delamination in Fiber-Reinforced Composites. The testing of fiberreinforced composite panels in aerospace applications has revealed a problem with delamination. Composites are designed and utilized to take advantage of their high strength in the plane of the fibers. Unfortunately, it is not possible to totally eliminate out-of-plane loading. One of the problems encountered in aerospace tests is impact damage during landing and takeoff. Such out-of-plane loading can lead to serious delamination because the organic matrix resins used to bind the fibers together in most composites are very brittle. This delamination can substantially reduce performance for both in-plane and out-of-plane loads. What makes this damage particularly troublesome is that it 
is often difficult to detect; once detected, it is difficult to assess how much the performance is affected; and if performance is seriously affected, delamination is often difficult to repair.

A number of studies have shown that when a matrix resin with improved fracture properties is used, the composite has significantly higher resistance to matrix cracking and other types of interlaminar fracture. Unfortunately, it is usually necessary to sacrifice other properties, such as modulus, to obtain improved fracture properties. Current work is investigating the use of rubber-modified epoxy resins. In these materials, the rubber is present as tiny particle-inclusions in a matrix of epoxy, and the two phase nature of these systems makes possible toughening mechanisms that cannot be achieved in single phase materials. As a result, these systems give very high fracture energies with a minimum sacrifice in other properties.

Fracture tests were conducted on neat resin specimens of modified and simple epoxies, and these tests show the large increase in fracture energy that can be obtained by rubber-modification. Both glass and carbon fiber reinforced composites were then fabricated with these resins and examined by propagating a crack in the matrix resin between the fiber layers. This test gives an interlaminar fracture energy which is one indication of the resistance of the composite to delamination. Such tests indicate that the interlaminar fracture energy of composites can be significantly increased by rubber-modification. Current work is aimed at developing a basic understanding of the mechanisms involved in composite failure and the factors which influence them. Such an understanding will provide guidelines for both improved composite designs and better materials.

Time-Dependent Fracture of Structural Adhesives. The high fracture energy of the rubber-modified epoxies has also made them the major constituent in most commercial structural adhesives. The mechanisms responsible for this high fracture energy are presently being studied with both bulk and adhesive bond specimens. A major result of this work is the demonstration that the toughening mechanisms are viscoelastic/plastic in nature, and thus, the fracture energy depends on the loading and temperature history of the sample. Experiments have shown that the fracture behavior for bulk samples of this material subjected to simple types of loading and temperature histories can be described with simple empirical equations. For example, over a range of conditions, the time to failure, $t_{f}$, in constant cross-head speed tests can be related to a parameter, $S_{E}$, that measures the rate of increase in strain energy during loading by a simple power law with an Arrhenius temperature dependence

$$
t_{f}=A\left(S_{E}\right)^{-n} e^{\Delta H / R T}
$$

Although this empirical equation only applies to a limited set of conditions, the parameters $A$, $n$, and $\Delta H$ are quite useful for comparing the relative rate and temperature sensitivity for different formulations. Studies are now underway to examine more general histories and models for failure of these commercially important materials.

\section{References:}

[1] G. I. Barenblatt, Adv. in Applied Mech., Vol. VII, Academic Press, New York, 1969.

[2] J. G. Williams and G. P. Marsha11, Proc. Roy. Soc. (London), A342, 55 (1975).

[3] D. S. Dugdale, J. Mech. Phys. Solids, 8,100 (1960).

\section{Instability and the Phenomena of Necking}

\section{J. Zapas, J. M. Crissman, B. Bernstein ${ }^{1}$, and B. D. Coleman ${ }^{2}$}

Failure of polymeric materials by neck formation and propagation depends on level of mechanical stress, temperature, as well as aspects of the molecular and physical structure. These relationships have been determined for polyethylene, a widely used thermoplastic. Analyses of these relationships have led to an empirical relation between the time required to reach a certain strain and the time at which a neck forms. For practical purposes, neck formation is identified as mechanical failure. Current work is aimed at developing models and experimental procedures to predict the instability point, that is, the time required for the material to reach the strain in question. Successful models and experimental procedures would provide a powerful method for predicting lifetime under conditions where neck formation is the mode of failure.

The Mechanical Behavior of Isotactic Polypropylene in Uniaxial Extension: Instability and the Phenomenon of Necking. A theory of stability for the deformation of viscoelastic bars which obey the BKZ theory [1] has been developed [2]. This theory is an extension of the one given by Ericksen [3] for elastic bars. For the appropriate strain potentials under certain loads in uniaxial creep and constant rate of extension, the bar will deform homogeneously for some time and then become unstable and neck. Ericksen's type of analysis has been applied to a viscoelastic situation in an effort to 
explain how time effects may enter into the onset of a discontinuity. While the theory does nut predict a priori when the material will neck, it does give an explanation for the formation of the neck, which depends on the stretch history, and also shows how a material may, when fully necked, have a higher modulus than it had originally.

From constant rate of strain experiments in uniaxial extension, we have found that a slowly quenched sample of isotactic polypropylene can be described rather well by the BKZ theory. By this, we mean that the stresses in a constant rate of strain experiment calculated using the BKZ theory were in good agreement with the experimental data. In all cases, the calculated values were lower than the observed values, but the maximum deviation was seven percent or less. This good agreement encouraged us to examine more closely the model proposed by Bernstein and Zapas concerned with the stability and cold drawing of viscoelastic bars.

A critical element in the application of the theory of Bernstein and Zapas is knowledge of the single step stress relaxation behavior. For semicrystalline polymers, such as isotactic polypropylene, single step stress relaxation data can be obtained only over a small region of strain. At strains above ten percent, the specimen necks during application of the step. However, the theory requires knowledge of such data over a much greater range of strain than is experimentally possible. Therefore, we have carried out a series of experiments involving a variety of different strain histories in uniaxial extension; these include single step stress relaxation, creep, constant rate of strain, and constant rate of loading. With the knowledge of the various strain histories, we have constructed one possible set of isochrones of stress vs strain by extrapolating our data into the regon of very high strains. From this set of curves and the instability theory, it is then possible to predict the point at which necking will occur for other strain histories.

We conclude that the Bernstein and Zapas instability theory may provide a reasonable description of the phenomenon of necking. This, we believe, may turn out to be a valuable procedure for predicting the incipient point of necking for even more complicated strain histories. The present work was undertaken as a first step for further investigation into understanding the phenomena of instabilities that may occur in other polymers.

Theory of the Instability and Failure of Viscoelastic Materials in Tension. In a tensile test of a bar or fiber formed from a transversely isotropic viscoelastic material, the initial motion in regions away from the clamps is a homogeneous uniaxial extension. If the applied tensile load is "tame" in the sense that it is given by a piecewise smooth function of time, then during the early stages of loading, the homogeneous exterision of the specimen is also tame. It is often the case that at a time $t_{c}$, previous to the instant of fracture, the motion departs from a tame homogeneous extension. The critical time $t_{c}$ is the "failure time" of the specimen, i.e., the time at which the motion first changes character; $t_{c}$ precedes, often by a constant factor, the time at which neck-down is easily visible. The problem of calculating $t_{c}$ for a given loading program has been treated within the framework of the general theory of non-linear simple materials with fading memory. For such materials, the instantaneous tensile modulus, i.e., the derivative of the immediate change in tensile stress with respect to a sudden change in tensile strain, depends upon the previous history of the strain. An explanation has been put forward for identifying $t_{c}$ with the earliest time $t_{0}$ at which the instantaneous tensile modulus becomes zero. It has been shown that at the time at which the instantaneous modulus vanishes, one cannot arbitrarily assign the rate of change of tensile stress and have the motion remain in the class of tame homogeneous extensions.

References:

[1] B. Bernstein, E. A. Kearsley, and L. J. Zapas, J. Res. Nat. Bur. Stand., B68, 103 (1964).

[2] J. L. Ericksen, J. Elasticity, 5, 191 (1975).

[3] B. Bernstein and L. J. Zapas, J. Rheology 25, 83 (1981).

\section{Test Method Development}

J. M. Crissman, D. L. Hunston, E. A. Kearsley, R. W. Penn, L. J. Zapas, G. M. Martin, and J. L. Rushford

Test methods are developed for evaluating mechanical performance and durability of polymeric materials. A joint program with the Department of Transportation led to the development of an improved test for environmental stress cracking of plastics. The method has been presented to the ASTM and test conditions are being optimized in current work prior to submission for round robin testing. A cooperative effort with DOE addresses the need for test methods to evaluate the long-term performance of elastomeric seals used in geothermal energy production. A test protocol has been devised that circumvents the problem of carrying out measurements in extreme environments similar to those found in the use conditions. 
A nondestructive measurement technique has been developed that permits dynamic mechanical testing and stress relaxation experiments as a function of temperature and time. The technique has been applied to the study of cure of composite materials and to the dependence of mechanical properties on aging time. The technique has application as a quality control method since an improperly prepared batch of thermosetting material can be easily detected.

Development of a New Test Method for Environmental Stress Crack Resistance. For thirty or more years, it has been recognized that ethylene-based plastics are susceptible to environmental stress cracking (ESC). This knowledge led to the rapid development of many test methods for evaluating environmental stress-crack resistance (ESCR), including those in most widespread use today. More recently, the use of ethylene-based plastics in the packaging industry has greatly expanded. One important area is that of hazardous materials transportation where because of increased energy constraints there is a demand for lighter weight shipping containers. The use of polyethylene in large shipping containers has been made possible in part by the development of new, more highly stress crack resistant polymer resins.

One result of this activity is that over the past several years there has been renewed interest in ESC and the development of new test methods which can, on a reasonable time scale, more adequately characterize ESCR. We have examined a number of test methods for ESCR and have developed one test which involves a bent strip geometry but differs from the current ASTM bent strip test in two ways: (1) all specimens are constrained in such a way as to maintain a constant geometry, and (2) the specimen is subjected to a constant applied load.

Tests are currently being carried out on three different polyethylenes having widely different ESCR. Two of the samples are linear polyethylenes having weight average molecular weights ( $M_{\text {) }}$ of approximately $10^{5}$ and $4.5 \times 10^{6}$, and the third is an ethylene-hexene copolymer having a $M_{w}$ of $W_{b o u t}$

$2.1 \times 10^{5}$. The test procedures being employed are intended to optimize the experimental conditions in order that data can be obtained in an acceptable time period for as wide a range of different polyethylenes as possible. Such procedures involve systematically changing the bend geometry, specimen thickness, magnitude of the applied stress, and temperature.

At the present time, the characterization of the two lower molecular weight samples is nearly complete. Work is continuing on characterization of the ultra high molecular weight material which requires very long times to collect data. It is anticipated that the data collection will be completed in about one year. Upon completion, a description of the test method as well as the appropriate statistical data will be submitted to ASTM Committee D-20.10 for their consideration.

Polymeric Sealants for Geothermal Energy. In a program to assist the Department of Energy to develop geothermal energy resources in the United States, we have been developing test methods for polymeric sealant materials to be used in the drilling of and producton of geothermal wells. Since there are many forms of seals used and down-hold environments vary considerably among the different well locations, it is not possible to specify reasonable requirements for geothermal sealants in general. The most interesting and energetically important wells are potentially those with ambient down-hold temperatures greater than $260{ }^{\circ} \mathrm{C}$ and with strongly corrosive, reducing (rather than oxidizing) atmospheres. The sealant materials should perform (frequently this means maintaining large elastic stresses for fixed deformations) under these demanding conditions for time periods ranging from hours for well-drilling sealants to months or years for production seals. Current technology for such seals is largely based on developments for the petroleum industry and is, consequently, adapted to lower temperatures and less corrosive conditions.

At present, various ad hoc lifetime tests are used by designers of geothermal equipment to test specific seals under conditions simulating the intended use. Such tests are very specialized and inconvenient. Failures of the seals are observed to occur either because the sealant hardens and eventually cracks or because the sealant softens and flows. The program on geothermal sealants in the Polymer Science and Standards Division aims at a more fundamental test which gets at the causes of this behavior so that the results can be used to design seals for whatever configuration or geothermal environment is needed. This is to be achieved by measuring the rates of formation and of breaking of bonds (cross-links or equivalent) using the method developed by Tobolsky, et al., to study oxidative degradation of rubber. In that method, two sets of measurements are required: (1) the stress relaxation of material aging while deformed and (2) the change in elastic modulus of material aging in an undeformed configuration. The latter data can be collected rather routinely, but stress relaxation is an inconvenient experiment, particularly under conditions corresponding to geothermal down-well atmospheres. Consequently, we have worked out the theory of a method to substitute permanent-set data for the stress relaxation data needed for the Tobolsky method. The theoretical work for this was completed previously.

Currently we have assembled apparatus in which we can age test samples in aqueous or dry atmospheres at pressures up to $3000 \mathrm{psi}$ and at temperatures up to $300^{\circ} \mathrm{C}$. The apparatus is constructed of stainless steel. Pressures and atmospheres are limited to the gases available in commercial cylinders. Test specimens can be held in various constant states of strain during their aging. Apparatus has also been developed to facilitate mechanical testing of the specimens without reclamping them. Our 
computer controlled hydraulic test system has been programmed to conduct the necessary testing efficiently and to reduce the mechanical properties data.

Determination of Mechanical Properties for Characterization, Quality Control, and NDE of Polymers Satisfactory performance of a polymeric material in a practical application is often directly related to the proper matching of the mechanical properties of the polymer to those required for the application over the range of temperatures and time scales involved. As a result, the measurement of mechanical properties can provide not only useful characterization data but also valuable quality control (QC) and NDE information. To obtain maximum benefit from mechanical property measurements, however, it is important that the behavior be determined over the widest possible range of temperatures and time scales (or frequencies). In this regard, a device has been constructed in cooperation with the Naval Research Laboratory to combine dynamic mechanical testing and stress relaxation experiments in a single instrument. This device provides data for low amplitude shear properties over an eight decade time scale range at temperatures from $-200{ }^{\circ} \mathrm{C}$ to $400{ }^{\circ} \mathrm{C}$. Samples can range from $70 w$ viscosity fluids to highly cross-linked solids. The instrument is extremely versatile and has already been used to examine fiber reinforced composites, structural adhesives, high temperature plastics, elastomers, propellants, plastisols, and printing inks. To illustrate the use of this device, three particular examples will be briefly discussed.

The problem of quality control for elastomeric materials is a difficult one because many ingredients are used and a number of processing steps are involved. As part of the manufacturing procedure, it is customary to make a number of small samples (ASTM plates) for each batch to serve as material for QC testing. Our test device has been adapted so that a small portion of an ASTM plate can be utilized to provide a detailed characterization of shear properties. With this information, an improperly prepared batch can easily be detected, and since the mechanical properties are related to molecular structure and morphology, it is possible in a number of cases to determine what mistakes were made in the formulation.

The nondestructive nature of this measurement technique is particularly helpful when the device is used to follow changes that occur in a material during cure. Two examples of this type have been studied during the past year. The Navy is interested in the properties of certain polyvinyl chloride plastisols for their studies of the interactions between turbulent flow and compliant surfaces. It was found, however, that the properties of this material change as a function of time. Our studies were able to provide not only a detailed characterization of the basic properties of these materials but also an analysis of how the properties change as a function of aging time. A second example of this type involves the printing inks used by the Bureau of Engraving and Printing for making currency. These inks cure during the printing process, and thus, good performance can only be obtained when the flow properties change in the required ways and at the proper rates during the cure. Studies are currently underway to characterize the properties of the inks as a function of cure so that better quality control techniques can be developed.

\section{Nonlinear Behavior of Polymeric Materials}

\section{J. Zapas, G. B. McKenna, and B. D. Coleman}

Constitutive relations are needed that adequately describe the nonlinear relationships found between stress and strain in most polymeric materials. These constitutive relations give connections between the macroscopic observables stress, strain, stress relaxation, etc., and are the basis for accelerated test methodology and lifetime prediction.

Nonlinear Elastic Behavior of Rubber Materials. In work carried out during the past year, we investigated the behavior of the derivatives of the strain energy function with respect to the stretch invariants, $\partial W / \partial I_{1}$ and $\partial W / \partial I_{2}$, of peroxide cross-linked natural rubber at deformations lower than those reported previously. In the course of our work, we examined the effects of the degree of crosslinking and of aging on the derivatives of the strain energy function. Our experiments were carried out in torsion, where both the torque and normal force were measured, to determine the strain energy function derivatives at values of the stretch invariants of $I_{1}=I_{2}$. In addition, we carried out experiments in simple extension and simple compression at strains as low as $\varepsilon= \pm 1.0 \times 10^{-4}$. By assuming the validity of the Valanis-Landel form of the strain energy function, we were able to relate the behavior in torsion with that in extension and compression.

The salient results of our work in torsion are:

(a) In no instance did we find that $\partial W / \partial \mathrm{I}_{2}$ became negative with decreasing strain; this is at variance with some results reported in the literature.

(b) The small strain torsional modulus, $G=2\left(\partial W / \partial I_{1}+\partial W / \partial I_{2}\right)$, is relatively unaffected by aging of the rubber, while the individual strain energy function derivatives, $\partial W / \partial I_{1}$ and $\partial W / \partial I_{2}$, change significantly.

(c) $\partial W / \partial I_{2}$ is relatively independent of cross-linking, while $\partial W / \partial I_{1}$ increases with increasing cross-linking. 
(d) At low degrees of cross-linking, $\partial W / \partial I_{1} \sim \partial W / \partial I_{2}$ for unaged samples. This result was unexpected.

The salient results of our work in extension and compression are:

(a) The apparent moduli in tension and compression at small strains are not equal.

(b) The values of the apparent modulus in tension and compression at very small strains does not become constant. Rather, as the deformation approaches zero, the modulus increases significantly.

Non-Linear Viscoelastic Behavior of Polymeric Materials. A new constitutive law which explains the differences between experimental results and currently existing theories for the nonlinear viscoelastic behavior of materials has been developed. The relationship between stress and strain for a shearing history can be written as:

$$
\sigma(t)=-\int_{0}^{\infty} f_{1}\left[\xi^{t}(s), s\right] d s+
$$

$$
\int_{0}^{\infty} \int_{0}^{\infty} f_{2}\left[\xi^{t}\left(s_{1}\right), \zeta^{t}\left(s_{2}\right) ; s_{1}, s_{2}\right] d s_{1} d s_{2}
$$

where $\zeta^{t}(s)=\gamma(t)-\gamma(t-s)$ and $\gamma(t)$ is the shearing strain. For all $s, s_{1}, s_{2} \geqq 0$ :

$$
\begin{aligned}
& f_{1}(0, s)=0 \\
& f_{2}\left(b, 0 ; s_{1}, s_{2}\right)=f_{2}\left(0, b ; s_{1}, s_{2}\right)=0 \\
& f_{2}\left(b, c ; s_{1}, s_{2}\right)=f_{2}\left(c, b ; s_{2}, s_{1}\right)
\end{aligned}
$$

Experimental work has been commenced to determine the material memory functions $f_{1}$ and $f_{2}$. This work is focusing on the torsional responses of epoxy and poly(methyl methacrylate). Single and double step stress relaxation histories are required to determine these material functions. Results for other histories, such as constant rate of strain, will be compared with predictions from the theory.

\section{Durability of Paper}

\section{J. C. Smith}

Paper used to make currency should have a good balance between durability and cost, as the replacement of worn out currency is an expensive operation. Because of this, the Bureau of Engraving and Printing supports work at NBS to identify and characterize those aspects of the morphology of paper networks which affect durability. The research results are expected to contribute to the development of durable paper made from blended wood pulps instead of the more expensive cotton and linen pulps presently used.

To achieve these objectives, it is necessary to have an improved understanding of the relationship between the structure of paper, the morphology of the fibers, the processing conditions, and the mechanical and physical properties of the paper and its components. Over the past several years, studies at the National Bureau of Standards on interfiber bondability and its relation to the forceelongation behavior of fiber networks have helped to provide some of this background information.

Tensile tests are performed on specimens cut from open-web handsheets of density $\sim 2.0 \mathrm{~g} / \mathrm{m}^{2}$. Experiments have shown that the resulting force-elongation curves fit an equation of the form

$$
F=A\left[\exp \left(x / x_{c}\right)-1\right]
$$

where $F$ is the force and $x$ is the elongation. $x_{C}$ is a scaling parameter having the dimensions of length, and $A$ is a parameter that serves as a modulus of elasticity. The two parameters, $A$ and $x_{C}$, are sensitive to the number of interfiber bonds per unit area in the specimens tested. Moreover, during a test $A$ and $x_{c}$ vary in value as the test proceeds, reflecting damage to the specimen and changes in the location of the bond breaks that occur. This possibility of characterizing the specimen at various stages of a test is expected to enable the calculation of a force level parameter representative of the average bond strength between fibers in a given test specimen. 
The average force at which a specimen fails by progressive bond breaks depends upon the density of bonds and upon the average bond strength. During a test, the density of bonds diminishes, but it is hoped that the $A$ and $x_{c}$ parameters can be used to compensate partially for this effect. Experiments to test this idea are in progress.

\section{Mechanical and Transport Properties of Polyethylene in Organic Solvents}

\section{J. C. Phillips and A. Peterlin}

Polymer films are being used as liners for landfills and chemical waste holding ponds to control the movement of hazardous materials. In order to develop data that can be used to estimate the useful lifetime of such polymer liners, we have measured the transport of an organic acid through low density polyethylene (LDPE) and have measured the mechanical properties of LDPE in the presence of an organic vapor. The organic solvents simulate the action of the landfill leachates or hazardous waste.

The preliminary phase of this work involved measurements of mass loss of acetic acid (HAc) in low density (LDPE) and high density bottles and sorption/desorption of acetic acid vapor in LDPE films. The mass loss measurements were performed at $40^{\circ} \mathrm{C}, 60^{\circ} \mathrm{C}$, and $78^{\circ} \mathrm{C}$. At the highest temperature ( $t=$ $78{ }^{\circ} \mathrm{C}$ ), mass loss for mixtures of $\mathrm{HAC} / \mathrm{H}_{2} \mathrm{O}$ were also done; the concentrations of $\mathrm{HAC}$ in volume percent were 100 percent, 75 percent, 50 percent, and 25 percent. From the mass loss-time data, the loss rate and time lag were determined for each density bottle. The former results, as a function of temperature, yield the activation energy of permeation, while the activation energy of diffusion was calculated from the latter results. The activation energy of permeation, $E_{p}$, was the same for each density bottle, and the activation energy of diffusion was uncertain due to errors in the time-lag determination. The loss rate data for the solutions of $\mathrm{HAc} / \mathrm{H}_{2} \mathrm{O}$ was found empirically to be a function of the volume fraction, $V$, of $H A C$, i.e., $\log Q=K+V^{\alpha}$, where $\alpha$ is a constant equal to 2.5.

The sorption/desorption data were obtained at $30{ }^{\circ} \mathrm{C}, 50{ }^{\circ} \mathrm{C}$, and $70{ }^{\circ} \mathrm{C}$ at a vapor pressure of $p \sim 17 \mathrm{~mm} \mathrm{Hg}$. The polyethylene film had a density of $\rho=0.906 \mathrm{~g} / \mathrm{cm}^{3}$ and a thickness of $10.16 \mathrm{x}$ $10^{3} \mathrm{~cm}$. Diffusivity and solubility as a function of temperature indicated, respectively, that the activation energy of diffusion and the heat of solution were roughly constant for the range investigated. The diffusion process seems to be Fickian with differences in sorption and desorption behavior which suggest a concentration-dependent diffusion coefficient.

This preliminary study has shown that the density of PE is a very important factor in making a choice of a specific liner material. The temperature effect and the volume fraction of HAc in the leachate are also essential parameters that influence the mass loss and loss rate.

Mechanical properties of LDPE were analyzed by separating the total strain, $\varepsilon$, into elastic, $\varepsilon$, and plastic, $\varepsilon_{\mathrm{pl}}$, components. These components were determined in air at $t \sim 23{ }^{\circ} \mathrm{C}$ from recovery measurements for imposed strains of $\varepsilon_{0}=9.0$ percent, 29 percent, and 54 percent by extrapolating $\varepsilon(t)$ data to $t=0$. The extension times or the time the sample was held, $t_{h}$, varied from 10 minutes to 30 days. As a function of $t_{h}$, the elastic strain decreases while the plastic deformation increases. If recovery measurements are performed in the presence of an organic vapor (e.g., ethyl acetate), $\varepsilon_{p \ell}$ becomes greater than its air or vacuum value while $\varepsilon_{\mathrm{e}}$ tends to become less.

A11 recovery data could be roughly represented on a single master curve by plotting a reduced recovery $\left[\varepsilon(t)-\varepsilon_{\infty}\right] /\left(\varepsilon_{0}-\varepsilon_{\infty}\right)$ vs the logarithm of a reduced time of $t / t_{h}$. The plastic deformation at infinite time, $\varepsilon_{\infty}$, is the permanent set of the material and also is a function of $\varepsilon$ and $t_{h}$. The recovery data obtained in a vapor environment at pressure p could also be plotted on the master curve by assuming a reduced time of $\left(t / t_{h}\right) f(p)$ where $f(p)$ was found empirically to be $1 /(1+\alpha p)$ with $\alpha$ being a constant.

The results of changes of $\varepsilon_{e}$ and $\varepsilon_{p \ell}$ with $t_{h}$ correlate very well with changes of transport (diffusivity and solubility) with $t_{h}$ and tend to emphasize the influence of the components of the total strain on the diffusion process. 
This task provides molecular and gas transmission standard reference materials (SRM's) to a $\$ 30$ B/ year plastics and elastomers industry. Molecular SRM's are used extensively in industry to calibrate gel permeation chromatographs (GPC's) which are used routinely to measure polymer molecular weights and molecular weight distributions. The latter are the most important physical properties in determining a polymer's processability and its ultimate performance.

Improved characterization techniques are essential to providing improved SRM's. A fundamental knowledge of polymer solution properties such as osmotic pressure, viscosity, diffusional behavior, and scattering power is essential to this goal. As a consequence, a majority of the activities in this task involve experimental and theoretical studies of polymer solution properties. As a spinoff of our characterization activities, this task also provides data and concepts which are useful in polymer processing. Some of these activities during the past year include:

- Certification of a new, narrow-molecular weight polystyrene standard, SRM $1479\left(M_{w} \sim 10^{6}\right)$.

- Continued development of a new state-of-the-art, seif-calibrating gel permeation chromatograph.

- Approximately 300 gas permeation experiments which have been carried out for the purposes of upgrading the certificate for SRM 1470 to include $\mathrm{He}, \mathrm{CO}_{2}$, and $\mathrm{N}_{2}$.

- Analytical and computer simulation studies of chain entanglements.

- Development and construction of a new state-of-the-art light scattering photometer. This instrument will have the option of static or dynamic light scattering measurements at elevated temperatures.

- Completion of a low shear rate rotational viscometer based on the floating rotor type (ZimmCrothers). Viscosities may be determined at shear rates as low as $3.0 \mathrm{~s}^{-1}$ and up to $8.0 \mathrm{~s}^{-1}$ at temperatures up to $150^{\circ} \mathrm{C}$.

- Completion of a theory of the phase behavior of polymer blends.

\section{Standards for Gas Transmission Measurements}

\section{J. D. Barnes}

Gas transport properties of polymeric materials are vital in assessing end-use suitability and durability of polymers in such applications as packaging and coating materials. Facilities similar to. the one developed at NBS should prove useful in quality control of processes for manufacturing film and sheet materials.

The NBS Automated Manometric Permeation Measuring Facility went into routine operation in February. Since that time, approximately 300 experiments have been performed. Each experiment represents a set of measurements at a given temperature and at a given upstream pressure, using one of four selected gases on three specimens. One hundred experiments are performed on each specimen of SRM 1470 for purposes of upgrading the SRM certificate to include $\mathrm{He}, \mathrm{CO}_{2}$, and $\mathrm{N}_{2}$ in addition to $\mathrm{O}_{2}$. The SRM certificate will also provide temperature and pressure coefficients for the time-lags and permeances. Figure 1 shows the pressure trace from a typical experiment. Note how, in the early phases of the experiment, sorbed gases from the previous experiment cause the pressure to rise in the cell even when no upstream pressure is applied. When this happens, the cells must be pumped out again. Upstream gas pressure is not applied until the pressure rise due to sorbed gases has fallen below an acceptable level. When the upstream pressure jumps from zero to the preset value (at the arrow in figure 1), there is no change in the trace until an induction period has passed. After a suitably long time, the pressure rises at a steady rate. The quantities characterizing the specimen are the induction period (time-lag) and the steady rate of pressure rise. Figures 2 and 3 give results for the rate of pressure rise and the time-lag of a typical specimen. The time-lag for He gas is less than one second and cannot be measured with our present setup.

The phenomenon of partial immobilization manifests itself when $\mathrm{CO}_{2}$ is used as the permeant species in SRM 1470. The time-lag decreases by about 20 percent at any temperature as the upstream pressure increases from $45 \mathrm{kPa}$ to $135 \mathrm{kPa}$. The shape of the time-lag pressure trace is also different in this case, but we do not yet have a solution to the transport equations which allow us to explicitly account for the contribution of partial immobilization when fitting data to the time-lag curve. 


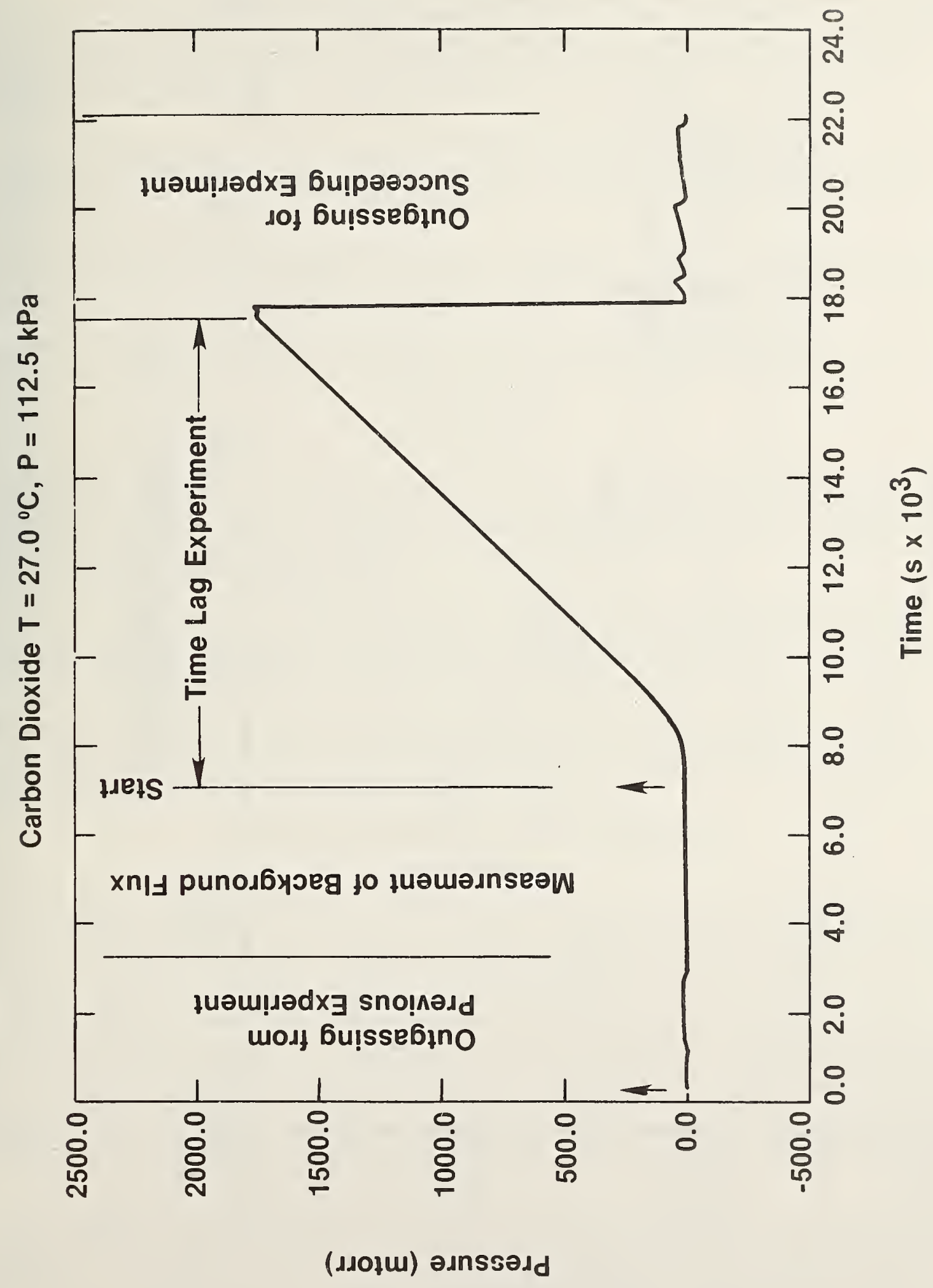




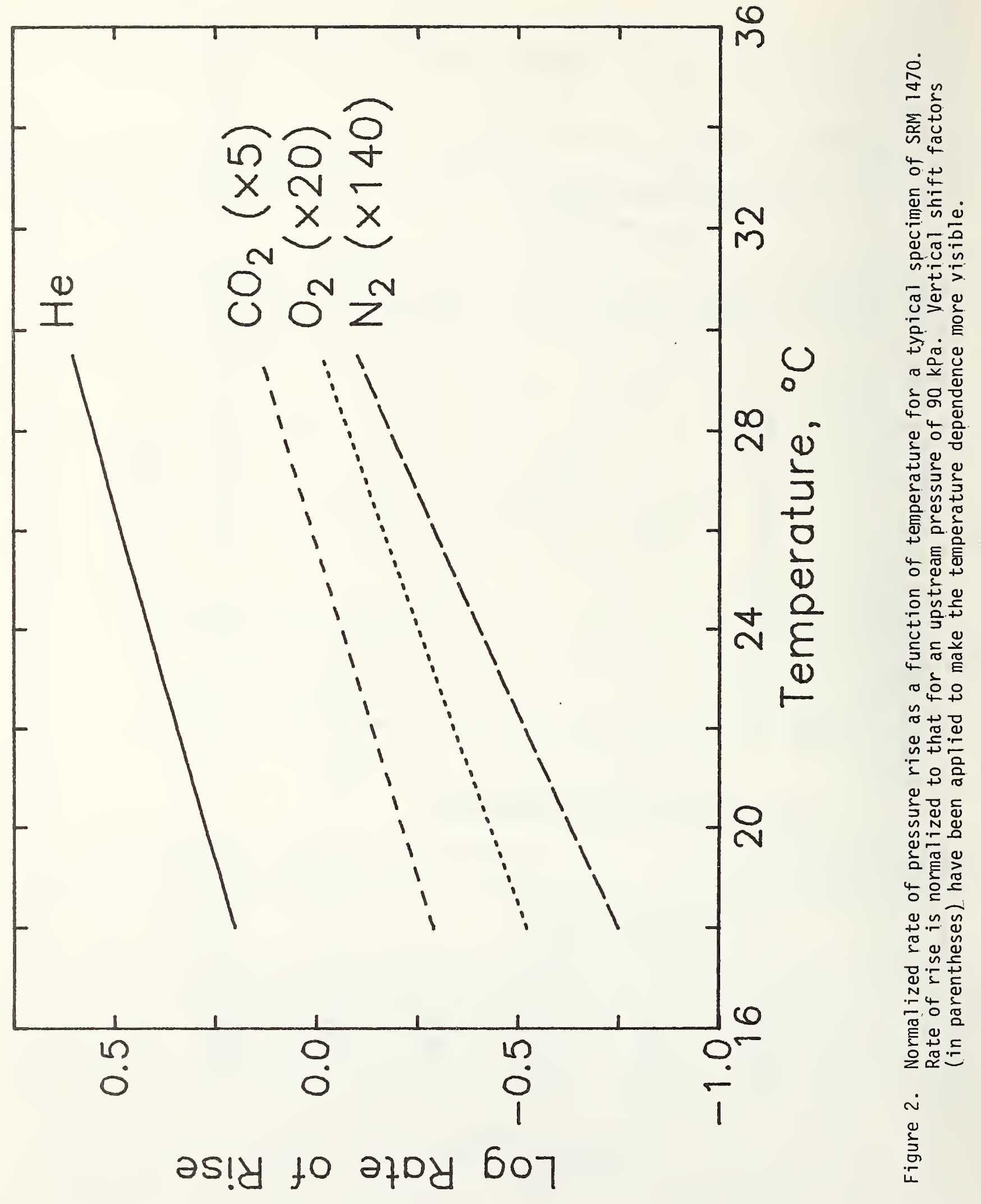


We found that data-taking totally occupied our apparatus and that it was necessary to send the data to the NBS central computer for reduction and analysis. In order to achieve this, we implemented a TIE into the NBS Net using software we developed ourselves. The data reduction programs have been copied into the 1108 and are presently being rewritten to take care of language and environment differences between the two machines. It is expected that all of the data for the new SRM 1470 certificate will have been analyzed by October 1 .

During FY82, we expect to finish upgrading the data acquisition hardware in the automated manometric permeation measuring facility. This replacement of antiquated MIDAS data acquisition equipment by more modern equipment using the IEEE-488 bus will allow us to study films with shorter time-lags and higher transmission rates.

We expect to undertake experiments near the glass transition temperature of SRM 1470 in an effort to better understand the role of molecular motions in transport processes. It is also believed that the glass transition plays an important role in regulating partial immobilization.

We expect to begin development of a simpler measurement scheme based on gas chromatography that will allow us to study interactions between permeant species in multicomponent mixtures.

\section{ASTM Activities}

\section{J. D. Barnes}

J. D. Barnes continues to serve as chairman of ASTM Subcommittees F-2.30 (Test Methods) and F-2.93 (Statistical Matters) of ASTM Committee F-2 on Flexible Barrier Materials. Subcommittee F-2.30 is responsible for test methods relating to materials characterization, barrier properties, sealing and bonding properties, and additive migration. Subcommittee F-2.93 provides statistical assistance to all groups needing it within Committee $\mathrm{F}-2$.

Dr. Barnes has drafted a Standard Practice for Selecting and Distributing Flexible Barrier Materials for Interlaboratory Testing Programs. This document is in the Subcommittee balloting stage, and it is expected that the adoption of this practice will enhance the ability of round-robin participants to obtain meaningful characterizations of the test methods they are studying.

Dr. Barnes is also a member of Subcommittees D-20.19 (Film and Sheeting), D-20.12 (01efin Plastics), D-20.70 (Analytical Methods), and D-20.13 (Statistics) of ASTM Committee D-20 on Plastics. Dr. Barnes has played a very active role in reviving interest in Subcommittee $0-20.13$, and the group receives many more requests for statistical assistance than formerly.

\section{Self-Calibrating Gel Permeation Chromatography}

\section{P. H. Verdier}

Gel permeation chromatography (GPC) is a widely accepted technique for estimating the molecular weight distribution (MWD) of high polymers. However, the usefulness of the conventional GPC apparatus is limited by the need to provide calibrants for each polymer measured of known molecular weight over the entire molecular-weight range in which the MWD is significantly different from zero. The calibration depends, among other things, upon the chemical nature, degree of branching, etc., of the polymeric material, so that each new material requires a fresh calibration. The so-called "universal calibration" hypothesis, while useful, is limited to comparisons of polymers of similar shape and, in any event, is inadequate for quantitative determinations. Some instruments, an example of which is commercially available, attempt to circumvent the need for calibrants by adding a single-angle light scattering detector to the usual concentration-sensitive detector. However, this does not allow the extrapolation to zero scattering angle which is required, in principle, to relate scattering intensity to molecular weight. In addition, qualitative information on the variation of scattering with angle, normally required to give assurance that meaningful results are being obtained, is not available.

We are designing and constructing a light-scattering detector for the GPC which measures, in real time, the scattering intensity as a function of scattering angle and which is controlled by a dedicated minicomputer in a way that allows scattering to be measured as a function of scattering angle and concentration. The instrument will allow continuous monitoring of the variation of scattering with angle. This will allow immediate identification of difficulties such as association, microgel formation, etc., which could affect the validity of the molecular weight obtained, an important consideration for work on new and unstudied materials. In addition to molecular weight, the mean-square radius (radius of gyration) will be obtained as a function of molecular weight, at least in the higher ranges of molecular weight, providing useful information for the characterization of branched polymers.

The mechanical components of the instrument have been constructed, and alignment is under way. The electronic components (preamplifiers, amplifiers, and switches) have been designed and prototypes built. In the coming year, we expect to complete alignment, construct the electronics, program the minicomputer and its associated video display, and begin taking measurements. 


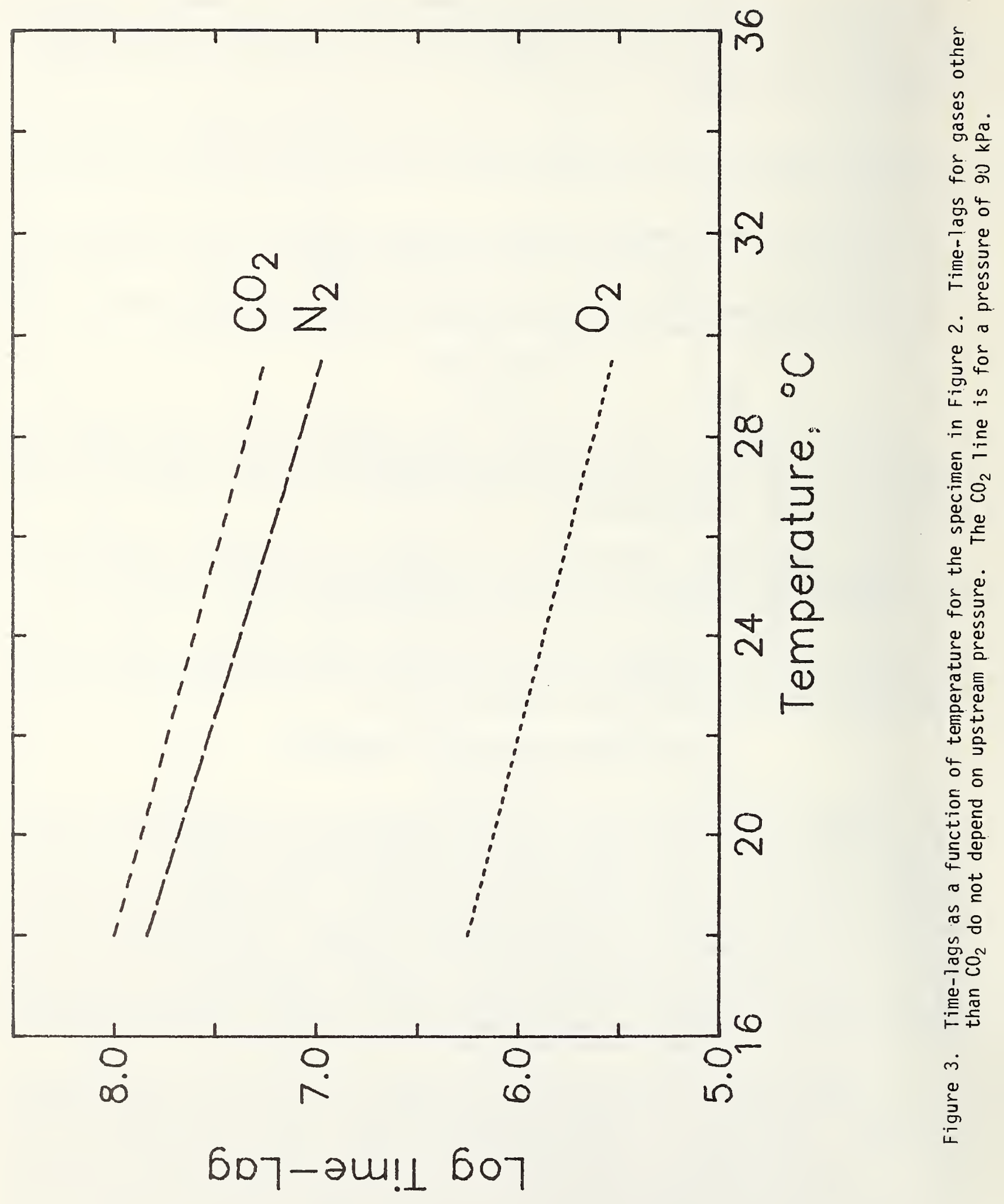




\section{P. H. Verdier and D. E. Kranbuehl}

The dynamic behavior of polymer chains is important to improving both characterization and processibility of polymers. The principal methods used to estimate molecular weight, and in particular gel permeation chromatography, the only method available at present for estimating distribution in molecular weight, are dynamical, non-equilibrium experiments which depend in part upon the relaxation of polymer chains in dilute solution. In its present state, the theory of these systems does not allow the calculation of molecular weight directly from measured quantities; a series of calibrants of known molecular weight is required. Improvements in the theory could reduce or even remove the need for calibrants, allowing the determination of molecular weight distribution of polymers for which calibrants are not available.

The processability and the final characteristics of both plastics and elastomers depend in large part upon the relaxation behavior of the high-polymer chains of which these materials are primarily composed. More realistic theoretical treatments of polymer melts and elastomers, which must start with more realistic treatment of the dynamical behavior of the individual chains, will lead to more efficient processing and better fabricated end-products. A major shortcoming of the present theory is its inability to treat chain entanglements in a realistic way. Our present work is aimed primarily at the study of entanglement effects, using a combination of analytical and computer simulation techniques. The simulation results provide data on simple model systems and serve to guide the development of better theoretical treatments.

Present work in this area is proceeding along three main lines. The first is a study of the effects upon our results of the necessarily crude models of polymer chains employed in our work. This study was prompted by the fact that our results show much larger effects of self-entanglement upon chain motion than are predicted by a currently popular theoretical model, which raises the question of the extent to which the effects we observe are artifacts of the models. We have extended our earlier studies of chain motion on several lattices to chains which are not constrained to lie on lattices. Perhaps surprisingly, the self-entanglement effects obtained for chains on lattices, which are largely independent of the lattice employed, become even more pronounced for chains free of lattice constraints. During the coming year, we expect to extend this work further by studying the motion of chains with move rules modified to provide increased local randomness at each step and to write up the resuits for publication. Second, there is at present an unresolved disagreement in the literature regarding the interpretation of the time-correlation functions obtained for flexible chain molecules in the quasielastic light scattering experiment. It is not clear whether these functions exhibit the entire spectrum of chain relaxation times or are dominated by the longest relaxation time. It appears that by direct simulation we may be able to resolve the disagreement, which will increase the value of measurements made by this relatively new technique. These studies have begun and should be completed in the coming year. Third, as a step in the direction of investigating melt properties, we have begun a study of systems of many interacting chains, with chain densities corresponding to concentrated solutions and melts. This work, which was partly supported this year by a grant from the NRCC, will be continued during the coming year.

\section{Low Shear Rate-High Temperature Rotational Viscometer}

H. L. Wagner and J. G. Dillon

Limiting viscosity number (intrinsic viscosity) is the most rapid and convenient method of measuring the molecular weight of high polymers provided the relationship between viscosity and molecular weight is known. However, for polymers greater than about a million in molecular weight, such as UHMWPE, the viscosity depends on shear rate as well as molecular weight. Hence, measurements should be made at zero or very low shear rate where this dependence is minimal and where, in addition, degradation due to shear is avoided.

A low shear rate rotational viscometer of the floating rotor type (Zimm-Crothers), which may be operated at the high temperatures required for polyethylene, has been designed and put into operation. Viscosities may be determined at shear rates as $10 \mathrm{w}$ as $3.0 \mathrm{~s}^{-1}$ and up to $8.0 \mathrm{~s}^{-1}$ at temperatures up to $150^{\circ} \mathrm{C}$. To assess the performance of the viscometer, measurements were made on several polystyrenes, two of which were Standard Reference Materials. The viscosity results were consistent with the previously published data on these samples, indicating that the instrument is operating satisfactorily.

Low shear rate limiting viscosity numbers for several samples of implant UHMWPE in decalin at $135{ }^{\circ} \mathrm{C}$ and a shear rate of $3.0 \mathrm{~s}^{-1}$ are shown in table 1, together with the results for these same polymers in capillary viscometers.

The multibulb capillary viscometer used here operates at shear rates of about $50 \mathrm{~s}^{-1}$ to $150 \mathrm{~s}^{-1}$; the standard type of capillary viscometer ordinarily employed for routine viscosity measurements

${ }^{1}$ Guest Worker, Food and Drug Administration 
provides a shear rate of about $2000 \mathrm{~s}^{-1}$. From the table it is seen that serious errors may occur if capillary viscometers are employed, even if extrapolation to zero shear rate is attempted using the multibulb viscometer.

A problem encountered in these measurements in the low shear viscometer is an initial rapid loss in viscosity of 2 to 3 percent during the first ten minutes of residence in the viscometer and as much as a 7 to 9 percent decrease in the first hour. This does not occur with the polystyrenes available to us. Work to understand and eliminate this difficulty is underway.

Table 1

Sample No.

99716

99881

$\underline{99974}$

Maximum Concentration

$0.046 \%$

$0.03 \%$

$0.02 \%$

Limiting Viscosity No.

Decal in $135^{\circ} \mathrm{C}$

(a) Zero shear rate (low shear viscometer, initial value)

$17.7 \mathrm{dL} / \mathrm{g}$

$24.2 \mathrm{dL} / \mathrm{g}$

$37.8 \mathrm{dL} / \mathrm{g}$

(b) Zero shear rate, estimated (multibulb viscometer)

18.9

34.0

(c) High shear rate (routine Canon-Ubbelohde viscometer)

14.6

17.8

26.0

Rubber and Rubber Compounding Standard Reference Materials

G. W. Bullman and G. B. McKenna

The process of streamlining the NBS Rubber SRM Program is continuing in response to reduced resources available for this program. As part of this reduction, rubber and rubber compounding Standard Reference Materials, for which NBS serves primarily as a warehouse facility, are being removed from the SRM list as inventories are exhausted. In addition, several materials are being removed from the 1 ist due to poor sales. These SRM deletions are being coordinated with ASTM Committee D-11 on Rubber and Rubber-Like Materials and Committee D-24 on Carbon Black to assure an orderly change in the ASTM standards and within the rubber industry.

In order to serve industry's needs for rubber SRM's with our reduced resource base, we are maintaining inventories of materials which industry (through ASTM) has indicated are important, even though NBS's primary function for these materials is to serve as a warehousing facility. For example, we recertified SRM 381 (N-tertiary-buty 1-2-benzothiazole sulfenamide, a rubber accelerator) in order to maintain inventories of this material. In addition, proposals were written to OSRM requesting working capital funds for the procurement of materials and recertification of SRM's 371 (Sulfur) and 372 (Stearic Acid) during FY82.

Where standards involve certification of fundamental physical properties, we are issuing new SRM's when a need is indicated. Thus, during the past year we issued a new Butyl Rubber, Mooney Viscosity Standard (SRM 1495) with a lower Mooney Viscosity Number than that available with the Butyl Rubber SRM 388. This new standard extends the range of Mooney Viscosity Standards available to the rubber industry.

\section{Static and Dynamic Configurational Properties of Polymer Chains: Theory}

F. L. McCrackin, R. J. Rubin, C. C. Han, J. Mazur, I. C. Sanchez, and D. J. Lohse 2

Comprehension of the configurational statistics of polymer chains is indispensable for a rational characterization of polymer solution properties. Constitutive properties of a polymer which are dependent upon its configuration include the intrinsic viscosity, mean square dipole moment, the optical anisotropy, and the spectral dichroism. Mechanical properties are also intimately related to the configurational characteristics of the polymer chain.

The hydrodynamic radii of polymers were computed for polymer chains at the theta point created by a Monte Carlo simulation in which both volume exclusion and the energetics of nearest-neighbor interactions are taken into account. The dimensionless ratios $\rho$ of the radius of gyration to the hydrodynamic radius were also computed. The calculated ratios of $\rho$ were compared with experimental ratios measured by elastic and inelastic light scattering. The calculated ratios showed better agreement with the

\footnotetext{
${ }^{2}$ Exxon Chemical Company
} 
experiment ratios than did values calculated for the analytcal Gaussian coil model of the polymer. The Monte Carlo calculations are being continued for different coordination numbers of the lattice of the polymers and for nearest-neighbor interaction corresponding to good solvents. The results are being compared to the blob model of a polymer and to available experimental data.

A review paper has been completed and is presently in the hands of the Washington Editorial Review Board of NBS. It is intended that this review be published in the series, Advances in Chemical Physics, edited by I. Prigogine.

In this review, the theory of discrete step random walks in discrete time and continuous time is outlined. The relation between continuous time random walks and a generalized master equation is developed. In the limit of long walks, the following asymptotic properties are discussed: (1) return to the origin, (2) number of distinct sites visited, (3) central limit results, and (4) walks with steps of infinite variance. The effect of boundary conditions and traps on random walk behavior, maximum displacements and spans, and the application of Wald's identity for first passage time problems is treated in a chapter devoted to boundary conditions.

A general method is outlined for calculating the statistical properties of the number of visits to a set of points in a random walk. In illustrative examples, a number of known results and new results are easily derived: (a) the generating function of the probability distribution of a random walk of $N$ steps with an excluded origin; (b) the generating function of the probability distribution of a random walk of $N$ steps to go from point $A$ to point $B$ with no intermediate visits to $A$ or $B$, $i . e$., a random walk with excluded origin and end point; and (c) the generating function of the probability of an $N$ step random walk to visit a surface layer of points $m$ times without ever crossing through the surface to the other side (i.e., penetrating the solution surface). This last result recovers a result obtained earlier by R. J. Rubin [J. Chem. Phys. 43, 2392 (1965)] for adsorption at a solution surface.

Mean-square radii of gyration were computed for comb-branched polymers simulated on a cubic lattice by chains with both excluded volume and attractive energies between non-bonded segments of the polymer. The ratios, $g$, of the radius of gyration of a comb-branched polymer of the same molecular weight were found to be larger than the g ratios calculated by the unrestricted random-wal < model of the polymer. The calculations were completed this year.

A statistical thermodynamic theory of a polymer solution is formulated which takes into account concentration inhomogeneities on size scales equal to or larger than the average size of a polymer chain. A generalized cell model is used as the basis for the new polymer solution theory. Closed-form, parametric equations are obtained for solvent and polymer chemical potentials which only reduce to classical (Flory-Huggins) potentials in concentrated solutions. The second virial coefficient $A_{2}$ varies as

$$
A_{2} \sim \frac{1}{M C^{\star}} \sim \begin{cases}M^{-1 / 5} & T \gg \theta \\ M^{-1 / 2} & T=\theta\end{cases}
$$

where $c^{*}$ is the overlap concentration (as defined in scaling theories) and $M$ is the molecular weight. For finite $M, A_{2}$ vanishes slightly below $\theta$. At $T=\theta$, the chains are still repulsive because of the existence of repulsive ternary monomer interactions. For the chain expansion factor $\alpha^{2}$, we obtain

$$
-c^{*} d \alpha^{2} / d c^{\circ}{ }_{c=0}=\text { positive constant; } T>\theta \text {. }
$$

This result implies that even in the dilute regime, chain dimensions should decrease with increasing concentration. The physics behind this result is that large fluctuations of the chain dimensions are damped because such fluctuations increase the probability of unfavorable interchain interactions. Neutron scattering experiments are being planned to check these predictions.

Reference:

[1] R. J. Rubin, J. Chem. Phys., 43, 2392 (1965).

\section{Polymer Blends}

I. C. Sanchez, F. W. Wang, and R. E. Lowry

Mixtures or blends of two or more different polymers can, under the proper conditions, produce materials with properties intermediate between those of the constituent polymers. This provides a method of tailoring a material to a specific application as well as optimizing the cost/performance ratio. Many plastic markets are primarily cost limited. Modern Plastics, a trade magazine says, "New polymer development is at a technological plateau. But new alloys--compounds of two or more structurally different polymers--are far more economical to develop than are generically new classes 
of plastics." Material costs are an important variable in the economic formula because the polymeric raw material is about two-thirds of the manufacturing cost of a typical plastic part.

A major generic problem associated with blends is the property that most polymer mixtures are incompatible and, given the opportunity, will phase separate. Although separated phases on a micro scale are often desirable, if the morphology and scale of the separated phases is not within acceptable limits, parts made from blends are useless. Since polymers tend to phase separate much more rapidly at the high temperatures encountered during processing, the phase separation problem is a matter of widespread concern which limits the rate of introduction of polymer blends.

Phase diagrams of liquid polymer/polymer mixtures (blends) are very unusual when compared to similar low molecular weight mixtures. Neither the familiar adage, "like dissolves like," nor the familiar observation that solubility increases with temperature are in general applicable to polymer blends. We have examined the thermodynamic and molecular reasons responsible for this unusual phase behavior. A general thermodynamic analysis of phase stability has been developed which suggests that thermally induced phase separation near a lower critical solution temperature is an entropy driven process. The entropic driving force is related to the compressible nature of a fluid mixture and the propensity of fluids to contract upon mixing (negative volume changes) at sufficiently high temperatures or, equivalently, sufficiently low fluid densities. By using a molecular equation of state theory, we have shown that volume contraction is always expected at low fluid densities if attractive interactions exist between dissimilar molecular species. All of the unusual aspects of polymer blend phase behavior can be given a rational basis.

There has been much effort to prepare polymer blends for the tailoring of polymers to specific end uses. A simple quantitative method for determining the point of polymer compatibility is needed. We have, therefore, initiated a project to develop a fluorescence energy transfer technique for the study of the phase behavior of polymer blends.

If the emission spectrum of a donor chromophore overlaps the absorption spectrum of an acceptor chromophore, nonradiative energy transfer can take place over distances as large as $5 \mathrm{~nm}$. The theory of this process is due to Förster who obtained for the efficiency, Eff, of the energy transfer on the donor-acceptor separation, $r, E f f=\left[1+\left(r / R_{0}\right)^{6}\right]^{-1}$ where the sixth power of the characteristic distance $R_{0}$ is proportional to the overlap integral.

Intermolecular fluorescence energy transfer will occur in a film made of two almost identical polymers, one labeled with a small amount of a donor chromophore and the other labeled with a small amount of an acceptor chromophore. In a blend made of these chromophore-labeled polymers and a third polymer, the efficiency of intermolecular energy transfer will be larger when the third polymer is incompatible with the first two and forms a separate phase than when it is compatible and serves as a diluting "solvent" for the first two. Thus, measurements of fluorescence energy transfer will allow us to determine the cloud point curve for the two practically identical labeled polymers and a third polymer.

We have synthesized donor- or acceptor-labeled polymers of narrow molecular weight distribution. We shall use the fluorescence energy transfer technique to determine the cloud point curve of these polymers and a third polymer.

\section{Static and Dynamic Scattering from Polymers}

C. C. Han, D. B. Minor, H. L. Wagner, A. Z. Akcasu³, S. J. Bai ${ }^{3}$, D. J. Lohse ${ }^{4}$, T. P. Lodge

Scattering techniques, such as light and neutron scattering, provide valuable characterization information on polymers. Static light scattering from a dilute polymer solution is the classical method of determining the weight average molecular weight of a polymer. It is a method widely used in industry for this purpose. Static scattering measurements also provide two other important pieces of information: the equilibrium size of the polymer molecule, i.e., its radius of gyration, can be determined as well as the osmotic pressure second virial coefficient. The latter is a thermodynamic property that is a useful indicator of the solvent power.

Dynamic or quasi-elastic light scattering can provide information on molecular motions, such as the diffusional and hydrodynamic behavior as well as information on equilibrium properties. In this respect it is a more versatile experimental tool than static scattering methods.

Much of our effort has been devoted to developing the idea that dilute polymer solutions satisfy a corresponding states principle. Polymer solution properties, such as intrinsic viscosity, are a function of three variables: molecular weight, temperature, and solvent. Scaling theories suggest that these three variables can be combined into a single excluded volume parameter and that all dilute

\footnotetext{
${ }^{3}$ University of Michigan

${ }^{4}$ Exxon Chemical Company
} 
solution properties can be expressed as a function of this single parameter. This corresponding states idea holds considerable potential for simplifying and unifying the different approaches used in polymer characterization. We have tested these ideas by comparing theory with experimental data collected from small angle neutron scattering, light scattering, and quasielastic light scattering measurements. To date, satisfactory agreement has been obtained which suggests that the corresponding states approach is valid.

A state-of-the-art light scattering photometer was developed and used in the measurement of polystyrene SRM 1479 at room temperature. However, because of the thermal instability of the optical bench used in this system, measurements at the elevated temperature, required for ultra high molecular weight polyethylene, could not be made. Consequently, the optical bench has been redesigned to be thermally isolated from the heated cell. In addition, a stepping motor driven goniometer has been installed. The redesigned apparatus will now how the option for simultaneous static and dynamic light scattering measurements at elevated temperatures that are required for polyethylene.

Polystyrene solutions in the dilute region have been studied by dynamic light scattering experiments. The first cumulant, $\Omega(q)$, has been extracted consistently by either cumulant analysis or shape function analysis. It is found that $\Omega(q)$ approaches $q^{2}$ dependence as $q R_{g} \ll 1$ and $q^{3}$ dependence as $\mathrm{qR}_{\mathrm{g}} \gg 1$ with a broad transition region. It is also found that the magnitudes of the first cumulant at $q^{3}$ region change from $\theta$-solvent to good solvent condition. In both cases, experimental results agree better with theoretical calculations which use a preaveraged 0seen tensor. It seems that either the analytical Gaussian model of equilibrium polymer chain distribution function or the 0seen tensor hydrodynamic interaction has to be improved. 


\section{J. M. Cassel}

\section{Task Leader}

The objective of this research is to contribute to health care through development of better performing materials and test methodology and standards appropriate thereto. Materials research and techniques are pursued with the goal of developing dental adhesive composite restorative materials of greater durability and wear resistance and improved base metal alloy alternatives to the costly gold alloy dental prostheses. Knowledge of the structure and physical chemistry of biologically important calcium phosphates is used to develop improved preventive dental techniques. Improved test methodology is sought for characterization of the surfaces of surgical implant materials for defining the interactions that occur thereon. Means for characterizing the porosity of low modulus, polymeric, medical implant materials are sought.

Dental research directions in support of the objective are established collaboratively with the American Dental Association, the National Institute of Dental Research, and the Department of Defense Medical R\&D Command. Medical materials research is guided by close interaction with the Food and Drug Administration's Bureau of Medical Devices and the ASTM F-4 Committee (Medical and Surgical Materials and Devices).

\section{Dental Composite Chemistry and Development}

G. M. Brauer, J. M. Antonucci, J. W. Stansbury, J. M. Cassel, R. L. Bowen ${ }^{1}$, H. Argentar ${ }^{1}$, D. N. Misra ${ }^{1}$, T. Chen ${ }^{2}$, and E. E. Toth

Current efforts are directed toward upgrading the quality of composite restorative materials with regard to color and storage stability, toxicity effects, adhesiveness, and durability.

Chemical and photo (visible and ultraviolet) polymerizations of experimental resin formulations in which the monomers, the amine or other type accelerator, and the initiator are varied are being compared for development of dental resin systems having enhanced storage stability and which yield dental composite restorative materials with improved color, chemical and mechanical stability, and are minimally toxic to the pulp. A number of mono- and dimethacrylate monomers have been prepared that are bulkier in structure than BIS-GMA and hence may produce less polymerization shrinkage in composite restorations derived therefrom. Various combinations of base resin, such as BIS-GMA, and new diluent monomers are being investigated for this purpose. Experimentation has shown that the extent of polymerization (or residual unsaturation) in the highly cross-linked dental composite resin systems can be investigated successfully by DSC. Correlation of DSC results with oxidative stability and wear resistance of composites is planned.

Photochemical polymerization-initiating systems that on the basis of their structure would appear biocompatible are being investigated. Means are being sought to effectively incorporate into clinically initiated paste/paste composite formulations non-amine type polymerization accelerators, such as ascorbic acid, which give composites with excellent color stability and biocompatibility when adsorbed onto the powder in powder/liquid systems. Amine accelerators which are esters of dimethylaminophenethanol and succinic, maleic, and acrylic acids have been synthesized and will be evaluated in composite resin formulations.

Continuation of the investigation of the effect of amines on the silanization of composite filler particles has shown that primary aliphatic amines are the most effective catalysts and aromatic and heterocyclic types the least effective. The solvent from which the coupling agent is deposited has also been shown to be an important factor. Non-polar solvents, such as cyclohexane, yield a higher degree of silanization with more resistance to hydrolysis than polar solvents such as acetone.

At least one manufacturer is preparing on a commercial scale the semi-porous glass developed earlier in this laboratory to achieve better reinforcement of the composite resin matrix by the filler component. The combination of an etched filler surface with the more effective means for applying the coupling agent will provide a means for achieving more durable composite restorations.

\footnotetext{
${ }^{1}$ Research Associate, American Dental Association.

${ }^{2}$ Guest Worker, Food and Drug Administration.
} 
G. M. Brauer, R. L. Bowen ${ }^{3}$, J. M. Antonucci, D. N. Misra ${ }^{3}$, J. W. Stansbury, E. Cobb ${ }^{4}$, J. M. Cassel

Although effective bonding to enamel is presently achieved by means of acid etch treatments, similar treatments of dentin are contra-indicated in tooth restoration procedures. A bonding procedure effective with dentin would promote more conservative tooth restoration procedures.

A series of so-called reactive adhesive formulations prepared by solubilizing selected elastomers in acrylic, methacrylic acid, or methacrylate ester monomers have been examined for their adhesiveness to mineralized tissue such as bone. Bond strengths after three months storage in water did not exceed 1. $0 \mathrm{MPa}$, and this approach has been discontinued.

While the stability to water of the bonding to dentin with isobutyl cyanoacrylate is not satisfactory, the initial bond strength attained with this material is the highest achieved to date. Attainment of this high initial bond strength requires a cleaning of the surface of the dentin by a one percent citric acid pretreatment but this is too severe for the dental pulp. However, pretreatment of the dentin surface with dilute sodium citrate solutions gave joints with excellent bond strength. To mitigate the response of the pulp to such materials, we have been examining the use of isotonic surface cleansing solutions to which we have incorporated mordanting agents as a means of altering or increasing the mineral surface to adhesion. Currently, we are examining the sequential application of two coupling agents, each applied from a volatile solvent. The coupling agents apparently penetrate into the altered dentin layer and provide bound molecules that can copolymerize with the resin of the subsequently applied composite material. The dentinal tubules do not appear to be enlarged or filled to any significant depth with the adhesive or polymeric material, so the very high bond strengths currently being achieved are not the result of mechanical attachment via resin tags penetrating the tubules and insulting the pulp. Other coupling agents that are designed to react with labile hydrogen groups in dentinal collagen have been synthesized and will be evaluated, e.g., monomers with pendant isocyanate and aldehyde groups.

Zinc oxide-eugenol ( $Z O E$ ) cements, because of the excellent biocompatibility of the hardened material, are widely used in dentistry. However, they have serious deficiencies: comparatively low strength, lack of resistance to wear, early disintegration, and inability to adhere well to acrylic restorations or to mineralized tissue. Further, their inhibiting effect on the free radical polymerization of acrylic resins and to a lesser extent dental composites with which they may be placed in contact, reduces their usefulness. In the past, substitution for the eugenol component which inhibits polymerization has usually resulted in cements possessing poor physical properties.

Currently, we are investigating cements in which the liquid ingredients are esters of vanillic acid or its isomers containing o-alkoxyphenol or other groups capable of forming chelates with multivalent salts. Neither vanillic acid nor its common isomers are liquids at room temperature, which is a prerequisite for conveniently preparing a cement by mixing the chelator with a metal oxide. The hexyl ester of vanillic acid (HV) was synthesized since it is a liquid at room temperature. The hardening reaction of this ester with zinc oxide is much too slow for dental application, but by incorporating a second chelating component such as o-ethoxybenzoic acid (EBA), we have increased the reaction speed within the useful range. The resulting cement has high strength, does not inhibit dental resin polymerization, and adheres strongly to acrylic resins and composites even after prolonged exposures in an aqueous environment. Incorporation of dimethacrylates in the formulations allows a rapid cure of such "composites" in the presence of an initiator-accelerator system and yields cements with good physical properties. Further improvement in these properties is being sought through the incorporation of divanillates.

The fact that HV-ZOE cements do not inhibit methacrylate resin polymerization has many advantages:

It allows incorporation of polymerizable monomers into the cement mix. By suitable techniques such as redox polymerization, these monomers can be cured to strengthen the cement and to yield crosslinked, multiphase, or interpenetrating networks which are less soluble, and more degradation resistant than the presently available cements.

A portion of the cement when used clinically as a temporary restoration can be retained as a base for an acrylic resin restoration. This should shorten the time necessary for placing the permanent restoration. A complete reexposure of deep cavities is also avoided, which should reduce irritation of the dental tissues during the clinical procedure.

\footnotetext{
${ }^{3}$ Research Associate, American Dental Association.

${ }^{4}$ Guest Worker, Georgetown University Dental School.
} 


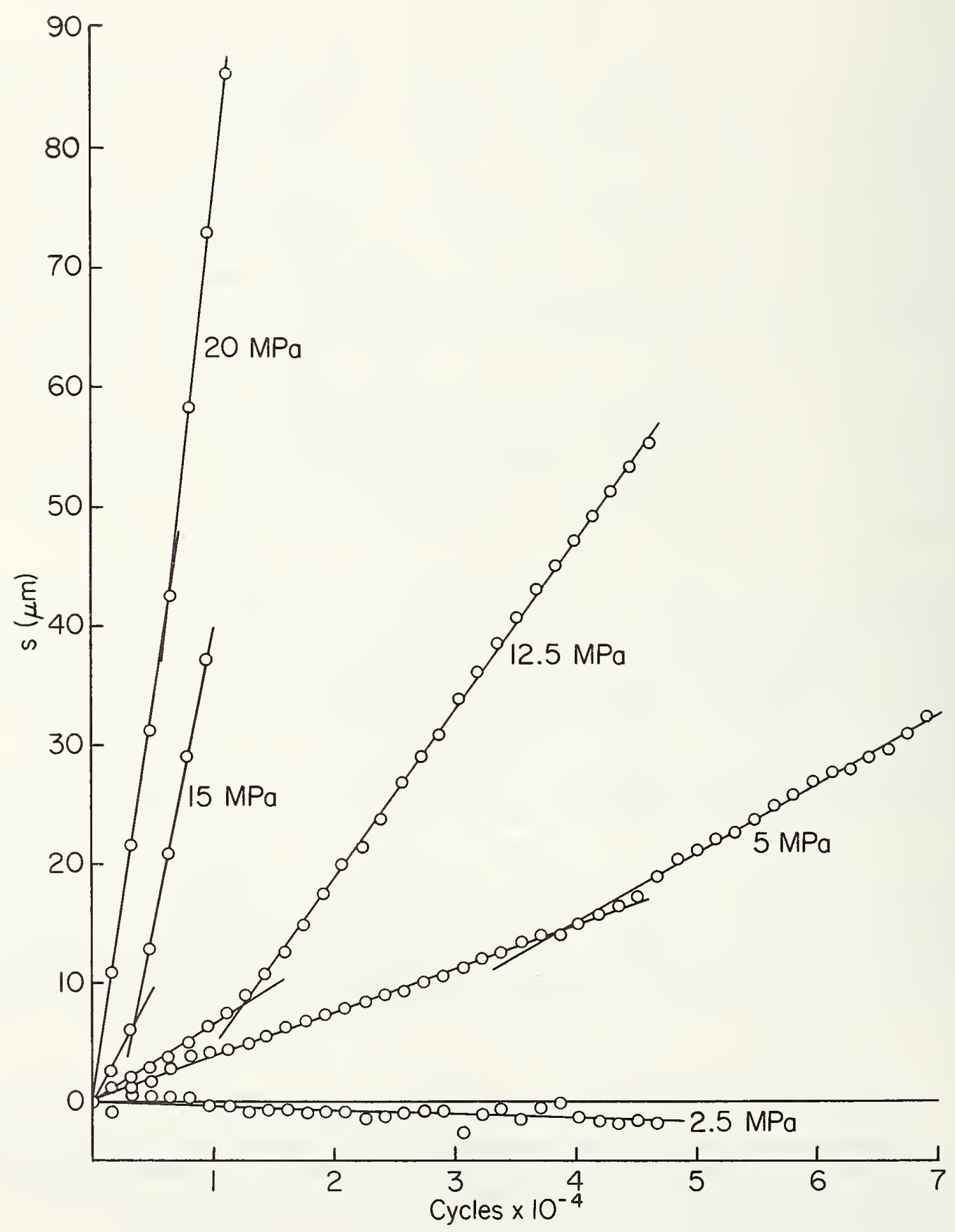

Figure 1. Wear track depth(s) for a dental composite plotted against specimen disc revolutions at indicated stresses. 
Wear Resistance and Mechanical Properties of Dental Materials

W. Wu, J. E. Mckinney, J. M. Casse 1, E. E. Toth, and E. Cobb

A silver staining technique developed and applied initially last year in a study of the mechanisms of wear in dental composite restorations is continuing to yield promising results. By this means, the creation of microdefected subsurface damaged regions is readily observed in both in vivo and in vitro worn specimens. This technique has been used to examine the attack of various solvent media including aqueous organic systems on dental composite materials and when combined with hardness measurements has shown that establishing solubility paraneter data on dental composite resins is important in investigating the mechanisms of degradation that influence wear resistance.

Small angle x-ray scattering experiments to examine the shape of the microdefects created in subsurface damage regions of worn composites are in progress. The necessary theoretical background for this application has been completed, and the analyses of in vitro compression-fatigued composite specimens are being compared with that of in vivo exposed materials. Preliminary results indicate a more isotropic-shaped defect in the clinical specimen with the conclusion that compression fatigue may not be a dominant factor in the in vivo wear process.

With our in vitro pin-on-disc accelerated wear test, we are continuing to advance our state of knowledge of composite wear. Analysis of composites subjected to wear over a range of normal stress levels from 2.5 to $20 \mathrm{MPa}$ have revealed that abrupt changes by a factor of roughly two in wear rate occur during the course of wear. This is shown in figure 1 where we plot the depth of the wear track as a function of the cycles of exposure of the dental composite disc to a stainless steel pin. We attribute this result to an initial wear region dominated by an adhesion process foilowed by a region in which subsurface damage increases to a point where breakdown occurs from fatigue. Only specimens with wear terminated in the second region of wear show subsurface damage by the silver staining technique. Subsurface damage caused by diffusion of solvents in dental composites is being correlated with in vitro wear performance using the silver staining technique. The extensive deterioration in the in vitro wear resistance of a commercial dental composite previously exposed to ethanol-water solutions is indicated in figure 2 where we plot the depth of wear track generated by a stainless steel pin in a dental composite disc after each of the first five revolutions of the disc. This type of information provides clues to efforts here and in industry to develop more durable plastic composite restorative materials.

\section{Porcelain Fused to Base Metal Alloys and Dental Ceramics}

\section{J. A. Tesk, R. W. Hinman 6 , W. deRijk ${ }^{7}$, E. E. Parry ${ }^{6}$, D. L. Menis ${ }^{7}$, and G. E. O. Widera ${ }^{8}$}

A major cause of failure in porcelain fused-to-metal restorations involves cracking or delamination of the porcelain veneer. This is thought to arise primarily from residual stress trapped within the system during cooling from the firing temperature. These stresses originate in the differences in thermal expansion and stress relief characteristics of porcelains and alloys.

To determine optimum combinations of thermophysical properties for porcelains and alloys, a multifaceted investigation of properties is in progress: (a) coefficients of linear thermal expansion are being determined for several brands of alloys and porcelains which have been subjected to one through seven laboratory firing cycles, (b) viscous flow characteristics are being measured, and (c) measurements of mechanical properties as a function of temperature are planned. Stress is being evaluated via a combined experimental and theoretical determination of the change in gap of porcelain veneered split metal rings using experimentally determined thermophysical properties. Correlation between measured and predicted gap changes provide the necessary information to calculate stress at any position within the ring. Ring and porcelain dimensions have been chosen to be somewhat representative of a crown.

Thermal expansion data for a number of porcelains have now been acquired and reported on. In addition to this property, information on the temperature dependence of the shear viscosity for a number of dental porcelains has been developed and reported on. A parametric study on the importance of parameters such as the temperature dependence of the elastic modulus and Poisson's ratio was conducted and discussed. Results of the latter study show that Poisson's ratio is not a critical factor in determining stress or strain within a system. The elastic modulus, however, is an important factor in stress. A 40 percent increase in modulus of porcelain can result in a doubling of stress within porcelains.

One area of particular concern involves the influence of transition bonding zones between alloy and porcelain on experimental determinations of stress and strain. Theoretical calculations of the effects of differences in thermal expansion showed that interfaces as thick as $20 \mu \mathrm{m}$, with differences

${ }^{5}$ Guest Worker, Georgetown University Dental School

${ }^{6}$ Research Associate, U.S. Navy

${ }^{7}$ Research Associate, American Dental Association

${ }^{8}$ Consultant, University of Illinois 


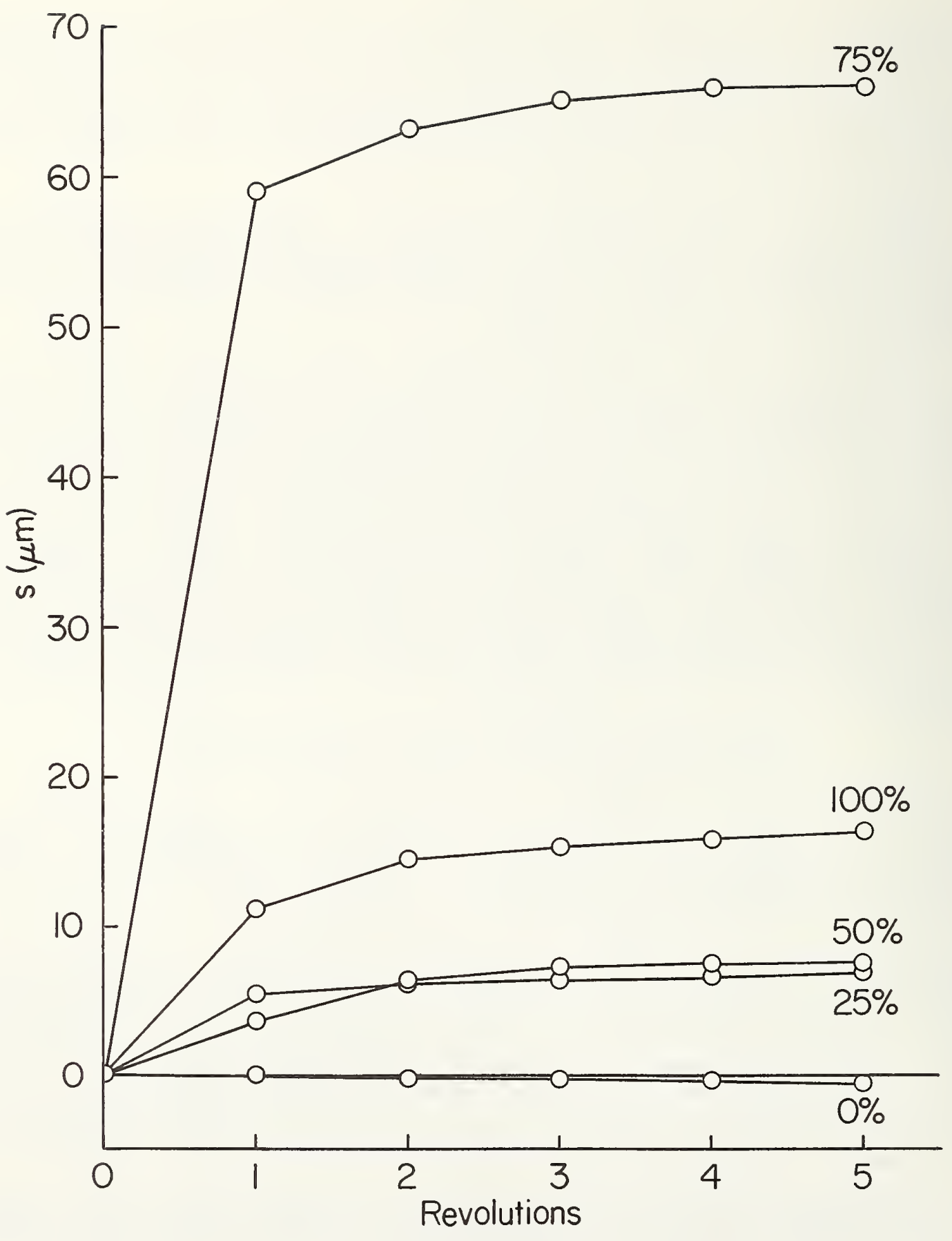

Figure 2. Wear track depth(s) for a dental composite measured over the first five revolutions. The specimens were immersed for two weeks in indicated concentrations of ethanol in water prior to wear tests. 
in expansion (from that of porcelain or alloy) as large as $10 \times 10^{-6} /{ }^{\circ} \mathrm{C}$, are not capable of producing significantly measurable effects at the 2.5 percent level of detection, provided the porcelain to alloy thickness ratio is one. Experimentally, this is the condition achieved in porcelain fused-tometal split rings. A paper was presented on this at the 1981 IADR/AADR meeting. It should be noted that the analysis used in this case is useful for predicting strain but is not capable of providing detailed stress analysis.

The theoretical approaches for calculation of stress in split metal rings has been refined; the finite element program has been reconstructed to include a finer mesh and definition of elements near edges (for edge effects) and to include viscous relaxation of porcelain. Stepwise numerical calculations, incorporating viscous flow, have been made using analytical models and have shown improved agreement with experimentally determined gap changes of composite rings.

The development of "Gel-route" technology for application to dental porcelain and investments has continued. "Ge1-route" technology, in which the desired composition of a solid phase is obtained by mixing aqueous solutions rather than by preparing a glassy frit has several advantages over conventional porcelain frit production; among them are lower capital and labor costs for production and less supply dependence on scarce raw materials. New compositional and heat treatment variations are being tested. Porcelain frits suitable for metal-ceramic applications have been made that mature in a 12 minute air fire at temperatures 50 to $175^{\circ} \mathrm{C}$ lower than the current lowest-fusing commercial metalceramic porcelain. The strength, thermal expansion coefficient, and insolubility (in four vol percent acetic acid solution at $80^{\circ} \mathrm{C}$ for one week) compare favorably with currently availabie materials.

A device has been constructed to measure the force of investment setting that uses the nondestructive setting-ring test developed for the measurement of both setting expansion and temperature set. Preliminary results using one phosphate bonded investment indicate that the force of setting is over four times greater with a wet asbestos liner than with an identical dry liner. It also increases slightly with increasing silica concentration in the liquid and with decreasing liquid-to-powder ratio. Most of the force, as well as expansion and heat evolved on setting, occurs after a hard set is achieved.

A patent on a microporous glassy filler for dental resin composites has been issued. Another patent on the "Gel-route" preparation of dental porcelain frit has been filed. Several dental companies have shown interest in our "Gel-route" prepared frit.

\section{Dental Casting Alloys}

\section{J. A. Tesk, R. M. Waterstrat ${ }^{9}$, R. W. Hinman ${ }^{10}$, and E. E. Parry 10}

Problems of cost and limited availability have been encountered in recent years for commercial dental casting alloys based on gold or cobalt, and some concerns continue to surface for the toxicity of nickel-based alloys, although there appears to be very limited evidence that this is indeed a problem except where a skin sensitivity to nickel has been established.

Earlier in this laboratory, we prepared the first titanium-based dental castings using a titanium/ copper alloy. These castings were made under argon with our conventional casting equipment. During the past year, we have designed and constructed an electric-arc furnace for melting and casting titanium alloys using conventional dental investment molds heated in the usual manner. The initial castings with this equipment demonstrate that this technique is feasible and may be more economical than the present induction centrifugal methods used in commercial dental laboratories. We have cast both the relatively low-melting titanium/copper alloys and pure titanium in the form of typical test castings. Much more information is needed relative to dimensional accuracy, mold materials, porcelainmetal bonding, mechanical properties, and casting techniques.

A study reported from this laboratory two years ago showed that single crowns of base meta? alloys could be cast to sufficient oversize to produce fits which would be considered as clinically acceptable. However, the question remains as to whether the same techniques would work for multipleunit castings. A series of experiments were, therefore, conducted to investigate the fit of multipleunit fixed partial dentures on a double die system which consisted essentially of combining two single dies of the pattern previously used. Results to date have shown that although some distortion of geometry occurred under all conditions investigated thus far, the use of plastic runner bars should be avoided and a bench setting technique with an all-wax system produced minimum distortion.

A pilot study attempting to join individual base-metal alloy castings by soldering has been unsuccessful to date. Alloys that performed best in our castability study often contain constituents which preclude reliable joining by conventional soldering methods. Some manufacturers who have been in contact with NBS are aware of the problem and are expending effort in this regard to improve their products.

${ }^{9}$ Research Associate, American Dental Association

10 Research Associate, U.S. Navy 
A simple, objective castability test which does not require sophisticated measurement devices would be of benefit to both users and developers of dental alloys. For this purpose, we had previously developed a technique that employs the use of a pattern of polyester mesh, waxed, sprued, and invested by standard dental laboratory practices. Following burnout of the pattern and casting of the alloy, the number of completed cast pattern segments provides a measure of castability. Results reported to the 1981 IADR/AADR meeting indicate that the test is sensitive to a number of processing variables such as casting temperature, mold burnout temperature, and investment brand. This development is being continued with a view toward evaluation of these parameters for a number of alloys and to make the test more sensitive to them.

\title{
Clinical and Laboratory Investigation of Dental Amalgams
}

N. W. Rupp ${ }^{11}$, G. C. Paffenbarger ${ }^{11}$, and R. M. Waterstrat ${ }^{11}$

The deterioration of amalgam restorations often begins with a slight extrusion from the cavity. This extrusion appears to be caused by some internal transformation which produces an expansion of the alloy. Recent experiments indicate the expansion is related to the amount of zinc in the amalgam, particularly in the higher temperature range $\left(60^{\circ} \mathrm{C}\right)$. Metallographic and $x$-ray diffraction studies have thus far been unsuccessful in identifying the cause of the expansion. Detailed metallographic studies of the reacted alloy powders are now underway, and further studies of the amalgams are to be initiated in the near future using a transmission electron microscope.

In a cooperative study with the University of Virginia, it was found that increasing the zinc content (1) increased the mercury/alloy ratio needed to obtain a proper consistency of mix, (2) increased the residual mercury content of the amalgam, (3) decreased dimensional changes that occur during hardening, (4) increased the compressive strength, and (5) decreased the creep.

Long-term clinical observations are continuing, and an attempt is being made to determine the causes of success or deterioration.

\section{Dental Chemistry and Preventive Dental Treatments}

\author{
W. E. Brown ${ }^{11}$, L. C. Chow ${ }^{11}$, M. Mathew ${ }^{11}$, M. S. Tung ${ }^{11}$, G. L. Voge ${ }^{11}$, \\ S. Takagi ${ }^{11}$, and T. M. Gregory ${ }^{11}$
}

The objective of this project is to develop and apply fundamental infornation on the solubilities and crystallographic structures of biologically important calcium-containing phosphate. compounds to the development of improved dental caries preventive techniques. Important portions of this effort are concerned with (1) development of physico-chemical models of the caries process to include insight into the mechanisms by which impurities and defects are incorporated onto tooth enamel, and (2) application of solubility phase diagram analysis and kinetic data to the development of more effective enamel acid-etch bonding treatments and improved topical fluoride procedures.

Continued progress has been made in developing a new procedure for studying the mechanism by which enamel crystals grow and react with fluoride. Advances were made in methodology, using rats as an animal model, as well as in collecting new experimental evidence. Considerable in vitro evidence has accumulated over the years that an acidic salt, octacalcium phosphate, is formed first in the developing enamel and then is converted to the final form, a basic salt, hydroxyapatite. Fluoride ions facilitate this conversion. Through the use of radioactive phosphorus, a promising new method was developed which measures the relative amounts of the acidic and basic calcium phosphates reproducibly in the rat incisor. The experimental results support the view that an acidic calcium phosphate is a precursor in tooth mineral formation, and they bring us a step closer toward understanding how fluoride in drinking water during tooth formation acts to reduce dental caries. These experiments were carried out in the animal facilities of the ADA Research Institute in Chicago.

Carbonate is a major impurity in tooth mineral, and its presence there is generally thought to enhance the solubility and, therefore, the caries susceptibility. Solubility measurements were carried out on synthetic and natural tooth mineral in the presence and absence of carbon dioxide in the atmosphere. Contrary to expectations, carbon dioxide lessened the solubility of tooth mineral. These results indicate that the effects of carbon dioxide in the atmosphere and of carbonate in the mineral on enamel solubility need to be examined more carefully in order to determine their roles in tooth decay.

Studies are being carried out on the chemistry of the tooth decay process by using ultramicro techniques. Small wells are drilled into quarter-miliimeter thick tooth sections. These sections are then placed into cariogenic solutions, and the chemical events taking place within the wells are measured. These wells are only about $0.1 \mathrm{~mm}$ in diameter and are barely visible to the unaided eye; since a complete chemical analysis of the solution within such wells must be obtained, it can be seen that the procedures involved are exceedingly delicate. Our recent work has shown that the solution

${ }^{11}$ Research Associate, American Dental Association 
within the wells is saturated with respect to enamel mineral and that the response time is relative: slow compared to the rates of change in the environment of the tooth due to normal dietary fluctuations These results require substantial modification of the caries models used in the past. Work of this type may reveal steps within the process that can be slowed so as to reduce the rate of formation of cavities.

Neutral salts such as $\mathrm{NaCl}$ and $\mathrm{KCl}$ through their ability to function as acids or bases have been predicted to strongly affect the caries process when the surface of the tooth holds either a positive or a negative ionic charge, respectively. This theory was tested in bench scale experiments and was found to be valid. The full implications of these results are not yet known, but the importance of neutral salts in the caries process warrants careful consideration in the future.

A previously reported topical fluoridation procedure which incorporates more permanently-retained fluoride into the tooth than the treatments now in use has been moved closer to clinical application. During the past year, several new modalities of treatment were tested in the laboratory and were found to be effective. Substantial progress was made in preparing for a clinical study of this procedure. This trial should go a long way toward confirming or refuting the commonly held hypothesis that increasing the fluoride content of enamel increases its resistance to decay.

We have determined the structures of several calcium phosphates with emphasis on iayer-type and highly hydrated structures. Two of these compounds, $\mathrm{CaBr}\left(\mathrm{H}_{2} \mathrm{PO}_{4}\right) \cdot 4 \mathrm{H}_{2} \mathrm{O}$ and $\mathrm{CaI}\left(\mathrm{H}_{2} \mathrm{PO}_{4}\right) \cdot 4 \mathrm{H}_{2} \mathrm{O}$, are of a new type in that they contain planar calcium phosphate sheets, a structure that has been found only once before. They provide a plausible structural model for calcium phosphate complexes in concentrated solutions. The structure of the compound $\mathrm{Ca}_{2} \mathrm{KH}_{7}\left(\mathrm{PO}_{4}\right)_{4} \cdot 2 \mathrm{H}_{2} \mathrm{O}$, which contains a corrugated sheet arrangement of calcium and phosphate ions, was determined, and work is in progress on a compound which apparently contains a calcium-phosphate sheet interlayered with urea molecules.

\section{Development of Improved Field Dental Composite Restorative Materials

for Military Personnel

\section{G. M. Brauer, N. W. Rupp ${ }^{12}$, J. W. Stansbury, K. Dermann ${ }^{13}$, and J. M. Cassel}

Field use of dental composites would be significantly aided by development of composite systems which could be more easily placed and have greater storage stability than current materials. As with all composite restorations, there is also the need for improved marginal adaptation in the hardened materials in order to prevent microleakage, which reduces the effective lifetime of restorations.

We have examined the roles in the phenomenon of microleakage of polymerization shrinkage, coefficient of thermal expansion, nature of the filler, and properties of diluent monomers. Based on radiotracer analysis applied to class $V$ restorations placed in extracted caries-free teeth that are subsequently thermocycled, we find the coefficient of expansion of a particular composite to be less important in microleakage prevention than is the influence of diluent monomer on the overall polymerization shrinkage. Measurements on specimens in which water sorbing diluent monomers have been incorporated to counterbalance polymerization shrinkage do not support this approach to solving microleakage problems.

A silver staining technique of investigative wear mechanisms of dental composites is being examined as a more sensitive means of assessing microleakage than the radiotracer technique we have been employing. The possibility of producing composite systems with low polymerization shrinkage but with a more hydrophobic character than that of currently available materials is also being investigated.

\section{Adhesion and Surface Coverage of Synthetic Polymers on Solid Substrates}

\section{R. E. Dehl and W. H. Grant}

Two aspects of the adhesion of polymers to surfaces are of great importance in their many commercial applications as protective coatings, namely, (1) the energy of adsorption of individual polymer molecules on the surface, which determines the adhesive strength of the film and (2) the extent of surface coverage by the polymer, which determines the effectiveness of the adherent film in protecting the underlying substrate from chemical attack. We are using radiolabeled polymers to study the adherence of the film. A measure of the energy of attachment of polymers adsorbed on solid surfaces is the rate at which they become detached when the polymer-coated substrate is placed in contact with a suitable solvent. The results of previous NBS studies, using radiolabeled polystyrene to detect the amount remaining on the surface, indicate that the rate of desorption decreases with increasing molecular weight of the polymer and with the amount of polymer initially adsorbed.

\footnotetext{
T2Research Associate, American Dental Association

${ }^{13}$ Freie Universität in Berlin
} 
We have recently discovered that the rate of desorption of polystyrene from a chromium oxide surface into cyclohexane solvent depends not only upon the amount adsorbed but also upon the way in which a given amount of polymer is adsorbed. If the time spent in adsorbing a given amount ( $A$ ) of polymer is very long or if the surface is first adsorbed to a higher level $B>A$, the subsequent rate of desorption into solvent from level A is much slower than if level A is attained quickly before desorption is begun. We feel that the most likely explanation for these phenomena is that the surface has at least two types of adsorption sites which differ in their energy of attraction for polymer molecules. We know that some adsorbed polymer exchanges with dissolved polymer during the adsorption so that a very slow adsorption allows the higher energy, less easily desorbed sites to fill with polymer at the expense of the lower energy sites. Similarly, adsorption to a higher level $B>A$ and desorption back to A leaves the surface with a higher fraction of high energy sites occupied than if the sites are quickly and randomly filled up to level A. The effects of surface roughness and other surface properties will be examined for their effect on the desorption rate of adsorbed polymers.

We are conducting studies to determine the utility of ESCA for determining the homogeneity of polymer coatings applied to solid surfaces.

\section{Characterization of Porosity of Porous Implant Materials}

R. E. Deh1, W. H. Grant, and J. M. Cassel

The use of porous synthetic implant materials to serve as scaffolding for the ingrowth of natural hard or soft tissue is a procedure utilized to stabilize selected medical implants. Characterization of the porosity of these materials is very important, since the porosity governs their successful utilization. In cooperation with the Bureau of Medical Devices, Food and Drug Administration, we have concluded a survey of published information and current commercial material specifications and have performed mercury porisimeter measurements on polymeric (polyethylene) and a composite (graphite fiber immersed in polytetrafluoroethylene) implant material. The results clearly demonstrate the usefulness of the mercury porisimeter technique for providing a "fingerprint" characterization of the materials.

A study of the effect of precompression on the composite material has shown that precompression by a pressure 20 percent greater than that encountered by the material during a mercury intrusion experiment did not change the ensuing pore size distribution curve. Deformation of the material at higher pressures sufficient to decrease the sample volume inelastically by 17,33 , and 67 percent changed the shape of the mercury intrusion curves significantly, indicating that the technique can be used to detect prior deformation of an unknown sample. In combination with a quantitative total void volume measurement, such as a continuation of $\mathrm{Hg}$ intrusion into the high pressure mode, this method can determine the fraction of total void represented by any pore size range of interest.

The accomplishment of this project's objectives will be of significance to the medical device industry, medical implant standards development, and, ultimately, the users of porous implant materials.

\section{Dental and Medical Materials Standards}

\section{J. M. Cassel, J. A. Tesk, G. M. Brauer, and N. W. Rupp ${ }^{14}$}

Dental and medical implant standards development is strongly supported by persons in positions of Committee, Subcommittee, and Task Force Chair roles in ANSI MD 156, Dental Materials, Instruments, and Equipment, and in ASTM F4, Medical and Surgical Materials and Devices. Additional leadership roles are fulfilled through the Chairpersons role in USA TAG ISO/TC 150, Surgical Implants and through participation in the Executive Committee of the ANSI Medical Device, Standards Management Board.

${ }^{14}$ Research Associate, American Dental Association. 


\section{Invited Talks}

A Computer-Controlled Manometric Permeation Measuring: Hardware and Software Considerations Washington Area MINC Users Group, Washington, D.C.

J. D. Barnes

January 15,1981

Effect of Matrix Toughness on Interlaminar Fracture of Composites

W. D. Bascom and D. L. Hunston American Chemical Society, New York, New York

August 24, 1981

Developments in Composite Resins

Howmedica Biomaterials Seminar, Groton, Connecticut

R. L. Bowen

September 24, 1980

Developments in Composite Resins

Academy of Operative Dentistry Annual Scientific Session, Chicago, Illinois

R. L. Bowen

February 12, 1981

Recent Developments in Adhesive Resin Formulations for Dentin

International Congress of Implantology and Biomaterials in Stomatology, Kyoto, Japan

R. L. Bowen

June 10,1980

Basis of Piezoelectricity and Pyroelectricity in Polymers

Symposium on Electroactive Polymers and Special Topics, Washington, D.C.

M. G. Broadhurst

January 9, 1981

Electrical Behavior of PVDF

Workshop on High Energy Density Capacitors, Maxwel1 Laboratories, San Diego, California

M. G. Broadhurst

April 28, 1981

Field Induced Phase Transitions of Polyvinylidene Fluoride

Gordon Research Conference, Ventura, California

M. G. Broadhurst

January 12, 1981

Dielectric Properties of Polyvinylidene Fluoride--Low Field and High Field Phenomena and Time Effects

Conference on Electrical Insulation and Dielectric Phenomena, Boston, Massachusetts

M. G. Broadhurst, G. T. Davis, and A. S. DeReggi

October 28,1980

Current Concepts in Dental Research

Georgetown University, Washington, D.C.

J. M. Cassel

January 7,1981

Application of Factor Jump Thermogravimetry to Polymer Degradation Studies

Eleventh Annual Summer Institute in Polymer Science and Technology, New Paltz, New York

B. Dickens

June 23,1981

Pressure as an Important Variable in Thermogravimetric Studies of Polymer Degradations

Second European Symposium on Thermal Analysis, Aberdeen, Scotland

B. Dickens

September 2, 1981

Solution Oxidation Studies With Styrene Polymers

Eleventh Annual Summer Institute in Polymer Science and Technology, New Paltz, New York B. Dickens

June 23,1981 
Thermal Degradation of Polyethylene and Polypropylene, Studied Using Thermogravimetry International Workshop in Thermal Analysis, Salford, England

B. Dickens

September 8, 1981

Overview of the Polymer Science and Standards Division

Environmental Protection Agency, Cincinnati, Ohio

R. K. Eby

September 26, 1980

Overview of the Structure and Properties Group

Environmenta] Protection Agency, Cincinnati, Ohio

B. M. Fanconi

September 26, 1980

Analysis of Thermogravimetric Kinetics--Overcoming Complications of Thermal History Symposium on Thermal Analysis in Polymer Characterization, New York, New York

J. H. Flynn

November 20, 1980

Kinetics of Polymer Degradation

Polytechnic Institute of New York, Brooklyn, New York

J. H. Flynn

May 13, 1981

Mettler Award Address

Tenth North American Thermal Analysis Society, Boston, Massachusetts

J. H. Flynn

October 27, 1980

Mettler Award Address

Royal Society of Chemistry, Salford, England

J. H. Flynn

September 7, 1981

New Techniques for the Thermal Analytical Investigation of Polymer Degradation Kinetics New Polymers and New Applications Symposium, Pittsburgh, Pennsylvania

J. H. Flynn

November 13, 1980

Role of Thermal Analysis in the Lifetime Prediction of Polymers

Second European Symposium on Thermal Analysis, Aberdeen, Scotland

J. H. Flynn

September 3, 1981

Thermogravimetric Analysis Kinetics

Symposium on Instrumental and Physical Characterization of Macromolecules of the Macromolecular Secretariat, Atlanta, Georgia

J. H. Flynn

Apri1 2, 1981

Dynamic Light Scattering From Dilute Polymer Solutions

University of California, Los Angeles, California

C. C. Han

January 14, 1981

Dynamic Light Scattering From Dilute Polymer Solutions in the Non-Asymptotic q-Region

University of Washington, Seattle, Washington

C. C. Han

January 19, 1981

Dynamic Light Scattering of Polymer Solutions in Dilute Region

Joint U.S.-France Symposium on Small Angle Neutron and X-Ray Scattering, Strasbourg, France

C. C. Han

September 23, 1980

Dynamic Light Scattering of Polymer Solutions in the Non-Asymptotic q-Region

State University of New York, Stony Brook, New York

C. C. Han

March 6, 1981 
Effects of Excluded Volume and Pre-Averaging Oseen Tensor for Dilute Polymer Solutions by Dynamic Light Scattering

University of Michigan, Ann Arbor, Michigan

C. C. Han

Apri1 7, 1981

Small Angle Neutron Scattering on Elastomer Networks

Gordon Research Conference, New London, New Hampshire

C. C. Han

July 14, 1981

Current Status of the Ceramometal Restoration

National Naval Medical Center, Bethesda, Maryland

R. W. Hinman

Apri1 1, 1981

Factors Influencing Selection of Fixed Partial Denture Alloys, Part I

Annual Meeting of the American Association of Dental Schools, Chicago, Illinois

R. W. Hinman

March 15, 1981

Current Concepts in Meta-Porcelain Systems

University of Texas, San Antonio, Texas

R. W. Hinman and J. A. Tesk

November 14,1980

Fracture Behavior of Brittle Materials

Workshop on Applied Rheology for Industrial Chemists, Kent State University, Kent, Ohio

D. L. Hunston

June 19,1981

General Rheology and Its Applications in Adhesion

Workshop on Adhesion and Adhesives, Kent State University, Kent, Ohio

D. L. Hunston

June 8,1981

Loading Rate and Temperature Effects in the Failure of Adhesives and Composites

Seventh SAMPE Symposium on Advanced Composites, Palo Alto, California

D. L. Hunston

January 16, 1981

Review of NBS and NRL Programs on Adhesion

Navy, Army, Air Force, and NASA Workshop on Adhesion, Dayton, Ohio

D. L. Hunston

Apri1 24, 1981

The Use of "Toughened" Polymers in Structural Adhesives and Composites

Martin Marietta Laboratories, Baltimore, Maryland

D. L. Hunston and J. L. Rushford

May 29, 1981

Fracture Toughness of the Polymers Used in Adhesive Bonding and Composites

D. L. Hunston, J. L. Rushford, J. L. Bitner, W. D. Bascom, and R. J. Moretton

American Chemical Society, New York, New York

August 24, 1981

Fluid Flow Problems and the Use of Polymeric Additives

Workshop on Applied Rheology for the Inudstrial Chemist, Kent State University, Kent, Ohio D. L. Hunston and R. Y. Ting

June 18, 1981

Elastic Strain Energy Functions

Illinois Institute of Technology, Chicago, Illinois

E. A. Kearsley

May 14, 1981

Measuring Elastic Strain Energies

Catholic University, Washington, D.C.

E. A. Kearsley

April 9, 1981 
Strain Energy Functions of the Valanis-Landel Type

Jet Propulsion Laboratory, Pasadena, California

E. A. Kearsley

November 17, 1980

The Diversity of the Habits of Polyethylene Crystals Grown From Solution

Cornell University, Ithaca, New York

F. A. Khoury

October 23, 1980

The Morphology of Uncrosslinked Polyethylene

Workshop on the Morphology of Polyethylene Power Cable Insulation, Gaithersburg, Maryland F. A. Khoury

March 3, 1981

Deformation and Failure Behavior of U1tra High Molecular Weight Polyethylene

University of Toronto, Toronto, Canada

G. B. McKenna

March 16, 1981

Failure Behavior of Polymeric Materials

Ecole Polytechnique de Montreal, Montreal, Canada

G. B. McKenna

March 12, 1981

Mechanical Durability of Candidate Elastomers for Blood Pump Applications

Toronto Division of the Canadian Society for Biomaterials, Toronto, Canada

G. B. McKenna

March 16, 1981

Adsorption of Benzoic Acid on Hydroxyapatite: Effect of Solvent and Hydrogen Bonding

International Association for Dental Research, Chicago, Illinois

D. N. Misra

March 19, 1981

Copper-Free Amalgams: Dimensional Changes After 4.5 Years

International Association for Dental Research, Chicago, Illinois

G. C. Paffenbarger

March 21, 1981

Dr. Fusayama's Books on Some New Departures in Operative Dentistry

American College of Dentists, New York, New York

G. C. Paffenbarger

September 9, 1981

Linear Dimensional Change for Zinc and Non-Zinc Containing Amalgams During Storage in Air at 60 , 37 , and $23{ }^{\circ} \mathrm{C}$

International Association for Dental Research, Chicago, Illinois

G. C. Paffenbarger

June 6,1980

Materials Used in Dental Health Therapy

University of Virginia, Charlottesville, Virginia

G. C. Paffenbarger

Apri1 27, 1981

Fracture Mechanics and Fracture Control Design

American University, Cairo, Egypt

E. Passaglia

Apri1 8, 1981

The National Cost of Corrosion and How its Magnitude is Measured

National Research Center, Cairo, Egypt

E. Passaglia

Apri] 5, 1981

Physical Testing of Polymers for Use in Circulatory Assist Devices

Fourth Annual Contractors Meeting, Bethesda, Maryland

R. W. Penn

December 3, 1980 
Mechanical Properties and Orientation in Polymers

University of Leeds, Leeds, England

A. Peterlin

Apri1 7, 1981

Brownian Motion, Polymer Adsorption, and Steady-State Enzyme Kinetics

California Institute of Technology, Pasadena, California

R. J. Rubin

March 10, 1981

Brownian Motion, Polymer Adsorption, and Steady-State Enzyme Kinetics

Stanford University, Palo Alto, California

R. J. Rubin

March 13, 1981

Effect of Solution Surfaces on Polymer Configurations

University of Groningen, Groningen, Netherlands

R. J. Rubin

June 24, 1981

Polymer Configurations and Boundaries

NBS Center for Applied Mathematics Colloquium on Mathematical Modeling in Science and Engineering,

Gaithersburg, Maryland

R. J. Rubin

October 29, 1980

Random Walks on Lattices: The Problem of Visits to a Set of Points Revisited

Twente University of Technology, Enschede, Netherlands

R. J. Rubin

June 17,1981

Transport of Radon in Porous Materials

Lawrence Berkeley Laboratory, Berkeley, California

R. J. Rubin

February 11, 1981

Achieving Excellence With Modern Dental Materials

Kootenai Study Club, Libby, Montana

N. W. Rupp

June 26,1980

Composites and Amalgams

Mid-Oregon Study Club, Bend, Oregon

N. W. Rupp

May 1, 1981

Cutting Tooth Tissues, Pulp Protection, and Microleakage

Georgetown University Dental School, Washington, D.C.

N. W. Rupp

February 26, 1981

Dental Amalgam

U.S. Navy Dental Center, Bethesda, Maryland

N. W. Rupp

March 30, 1981

Indirect Restorative Materials

Veterans Administration Hospital, Washington, D.C.

N. W. Rupp

March 30, 1981

Manipulation of Dental Materials

Veterans Administration Hospital, Washington, D.C.

N. W. Rupp

February 5, 1981

Materials for Indirect Restorative Dentistry

Navy Dental School, Bethesda, Maryland

N. W. Rupp

January 12, 1981 
Materials for Removable Prosthodontics

Veterans Administration Hospital, Washington, D.C.

N. W. Rupp

November 6, 1980

Materials for the Laboratory Technician

Veterans Administration Hospital, Washington, D.C.

N. W. Rupp

Apri1 28, 1981

Modifications in Cavity Preparation Using Acid Etch Techniques

American Dental Association Annual Scientific Session, New Orleans, Louisiana

N. W. Rupp

October 14, 1980

New Developments in Amalgam and Composites

Montgomery County Study Club, Gaithersburg, Maryland

N. W. Rupp

November 20, 1980

New Developments in Composites

Dental Study Club, Washington, D.C.

N. W. Rupp

October 27, 1980

New Developments in Restorative Dentistry

Navy Dental School, Bethesda, Maryland

N. W. Rupp

0ctober 23, 1980

Prosthodontic Materials: Selection and Use of Amalgam and Composites

Temple University, Philadelphia, Pennsylvania

N. W. Rupp

January 7,1981

Porcelain-to-Metal Restoration and Impression and Die Materials

D.C. Progress Study Club, Washington, D.C.

N. W. Rupp

January 26, 1981

Recent Developments in Dental Materials

Dentsply International Inc., York, Pennsylvania

N. W. Rupp

July 23, 1980

Restorations of the Future

Academy of Fold Foil Operators, New Orleans, Louisiana

N. W. Rupp

October 10, 1980

Selection and Manipulation of Dental Materials

Wilford Hall Dental Center, San Antonio, Texas

N. W. Rupp

August 7,1980

Selection and Use of Dental Materials

Eglin Air Force Base, Florida

N. W. Rupp

September 23, 1980

Selection and Use of Dental Materials

Southern Maryland Dental Society, Silver Spring, Maryland

N. W. Rupp

December 1, 1980

Selection and Use of Dental Materials

Barksdale Air Force Base, Shreveport, Louisiana

N. W. Rupp

February 24, 1981 
Selection and Use of Dental Materials

Sheppard Air Force Base, Wichita Falls, Texas

N. W. Rupp

March 10, 1981

The Selection and Use of Dental Materials

St. Louis Dental Society, St. Louis, Missouri

N. W. Rupp

April 6, 1981

Selection and Use of Dental Materials

offutt Air Force Base, Omaha, Nebraska

N. W. Rupp

May 20, 1981

Selection and Use of Dental Materials for the General Dentist

Oregon Study Club, McMinnville, Oregon

N. W. Rupp

May 4, 1981

The Selection of Composite Restorative Materials and Their Proper Use Georgetown University Dental School, Washington, D.C.

N. W. Rupp

April 2, 1981

Selection of Materials and Handling to Prevent Microleakage

Georgetown University Dental School, Washington, D.C.

N. W. Rupp

March 26, 1981

Selection of Prosthodontic Materials

Georgetown University Dental School, Washington, D.C.

N. W. Rupp

March 12, 1981

A Mean Field Theory of Polymer Solutions

International Symposium on Phase Transitions in Polymers, Cleveland, Ohio

I. C. Sanchez

June 11,1980

Equation of State Theories of Low and High Molecular Weight Liquid Mixtures

Exxon Corporate Research Laboratories, Linden, New Jersey

I. C. Sanchez

July $7,1.981$

Equation of State Theories of Polymer Blends

Centre de Recherches sur les Macromolecules, Strasbourg, France

I. C. Sanchez

october 23, 1980

Equation of State Theories of Polymer Blends

University of Texas, Austin, Texas

I. C. Sanchez

November 25,1980

Equation of State Theory of Polymer Blends

Center of Molecular and Macromolecular Studies, Lodz, Poland

I. C. Sanchez

October 28,1980

Molecular Origins of LCST Behavior in Polymer Solutions

CEN de Saclay, Gif-sur-Yvette, France

I. C. Sanchez

October 21,1980

Polymer Collapse Transitions

Institute of Polymer Chemistry, Zabrze, Poland

I. C. Sanchez

October 27,1980 
Polymer Collapse Transitions

Institute of Fundamental Technological Research, Warsaw, Poland

I. C. Sanchez

October 30,1980

Polymer/Polymer Interactions in the Dilute Regime

American Physical Society, Phoenix, Arizona

I. C. Sanchez

March 16, 1981

Statistical Thermodynamics of Fluid Mixtures

Ecole Nationale Superieure des Mines, Paris, France

I. C. Sanchez

october 20, 1980

Surface Thermodynamics of Liquid Polymers: Theory

International Symposium on Physicochemical Aspects of Polymer Surfaces, New York, New York

I. C. Sanchez

August 23,1981

Theory of Interfacial Tension in Polymer Mixtures

McGill University, Montreal, Canada

I. C. Sanchez

December 4, 1980

Diffusion and Transport in Polymers

University of North Carolina, Raleigh, North Carolina

L. E. Smith

Apri 1 1, 1981

Models for the Diffusion in Polyolefins

New York Academy of Sciences, New York, New York

L. E. Smith

May 27,1981

A Planar Ca- $\mathrm{PO}_{4}$ Sheet Structure: $\mathrm{CaBrH}_{2} \mathrm{PO}_{4} \cdot 4 \mathrm{H}_{2} \mathrm{O}$

International Association for Dental Research, Chicago, Illinois

S. Takagi

March 20, 1981

Compatibility of Dental Porcelains and Alloys

Johnson and Johnson Research Laboratories, East Windsor, New Jersey

J. A. Tesk

May 11,1981

Consideration of Some Factors Influencing Compatibility of Dental Porcelains and Alloys

Fourth Annual Meeting of the International Precious Metals Institute, Toronto, Canada

J. A. Tesk, R. P. Whitlock, G. E. O. Widera, A. D. Holmes, and E. E. Parry

June 8,1980

Applications of High Resolution ${ }^{13} \mathrm{C}$ NMR Techniques to Polymers

Akron University, Akron, Ohio

D. L. VanderHart

October 3, 1980

Applications of High Resolution Solid-State ${ }^{13} \mathrm{C}$ NMR in Polymers

Allied Chemical Corporation, Morristown, New Jersey

D. L. VanderHart

November 20, 1980

${ }^{13} \mathrm{C}$ NMR in Oriented Polymeric Solids

Twenty-Third Rocky Mountain Conference for Applied Spectroscopy, Denver, Colorado

D. L. VanderHart

August 3, 1981

Expectations for Resolution in Solid-State ${ }^{13} \mathrm{C}$ NMR of Polymers

Bell Laboratories, Murray Hi11, New Jersey

D. L. VanderHart

November 21,1980 
Inferences About Molecular Motion From Proton-Decoupled ${ }^{13} \mathrm{C}$ NMR Spectra of Polymers

American Chemical Society, New York, New York

D. L. VanderHart

August 25, 1981

Resolution in Solid-State ${ }^{13} \mathrm{C}$ NMR of Polymers

Monsanto Chemical Company, St. Louis, Missouri

D. L. VanderHart

December 8, 1980

Wear of Dental Composites

U.S. Public Health Service Hospital Seminar, San Francisco, California

W. L. Wu

January 26,1981

Some Aspects of the BKZ Elastic Fluid Theory

Catholic University, Washington, D.C.

L. J. Zapas

March 26, 1981

Seminars for Staff and Guests

The Chemistry and Structure of Guayule Rubber

Donald McIntyre

University of Akron, Akron, Ohio

June 3, 1980

Consideration of Some Factors Influencing Compatibility of Dental Porcelains and Alloys John A. Tesk

National Bureau of Standards, Washington, D.C.

June 30,1980

Electron Beam Probing of Charge Distributions in Polymer Films

David W. Tong

General Electric Company, Schenectady, New York

July 10,1980

Dielectric Behavior of Vinylidene Fluoride-Tetrafluoroethylene Copolymers

Naokazu Koizumi

Kyoto University, Kyoto, Japan

September 3, 1980

Simulation of Polymer Dynamics

Marshall Fixman

Colorado State University, Fort Collins, Colorado

October 6, 1980

Non-Linear Viscoelasticity of Polymers and the Fracture Behavior by Finite Element Analysis Shiro Matsuoka

Bell Laboratories, Murray Hill, New Jersey

October 6, 1980

Chemical Kinetics in the Hydrolytic Degradation of Polyester Based Polyurethanes

Daniel W. Brown

National Bureau of Standards, Washington, D.C.

October 8,1980

High Modulus/High Strength Organic Fibers: Spinning and Mechanical Properties

W. Bruce Black

Monsanto Textiles Company, Pensacola, Florida

October 29, 1980

Influence of Internal Structure on Small Deformation Properties of Crystalline Polymers James Sefaris

University of Washington, Seattle, Washington

October 29, 1980

The Role of Chemical Bonding in the Adhesion of Polymers

Alan N. Gent

University of Akron, Akron, Ohio

October 31, 1980 
Crystallization Kinetics of cis Polyisoprene at High Pressures

Eddy N. Dalal

University of Utah, Salt Lake City, Utah

November 6, 1980

Healing of the Polymer-Polymer Interface by Reptational Motion

Stephen Prager

University of Minnesota, Minneapolis, Minnesota

November 7, 1980

Frequency Dependence of Remanent Polarization and the Correlation of Piezoelectric Coefficients With Remanant Polarization in PVDF

J. Charles Hicks

Naval Ocean Systems Center, San Diego, California

November 10, 1980

FTIR Characterization of Conformational Changes in Amorphous Poly(Ethylene Terephthalate) During Volume Recovery

Robert S. Moore

Eastman Kodak Company, Rochester, New York

November 13, 1980

Light and Neutron Scattering Studies of Concentrated Polymer Solutions

Jeffrey T. Koberstein

Princeton University, Princeton, New Jersey

November 14, 1980

New Substituted Polyethers and Polysulfides via Ring Opening Polymerization

David Tirrell

Carnegie-Mellon University, Pittsburgh, Pennsylvania

November 17, 1980

Fracture Mechanics of Plastic Products

Michael D. Taylor

Manchester Polytechnic, Manchester, England

November 18, 1980

Polymer Compatibility and Phase Transitions

Ludwik Leibler

Centre de Recherches sur les Macromolecules, Strasbourg, France

November 21,1980

Fluid Phase Equilibria

Jean-Louis Guillevic

Ecole Nationale Superieure des Mines de Paris, Paris, France

November 24, 1980

Dependence of Crystalline Morphology of Polyethylene Fractions on Molecular Weight and Crystallization Temperature

Ingrid Voigt-Martin

Johannes-Gutenberg-Universität, Mainz, West Germany

November 25, 1980

Fluorescence Studies of Polymer Conformation and Chain Dynamics

Mitchell A. Winnik

University of Toronto, Toronto, Canada

December 11,1980

Applications of Finite Element Modeling in Dentistry

Otto Widera

University of Illinois, Chicago, I1linois

December 15, 1980

Irreversible Deformation Processes in Amorphous Polymers

Eric Baer

Case Western Reserve University, Cleveland, Ohio

December 30, 1980 
Reversible Crystallization and the Thermodynamics of Piezoelectricity in PVDF Philip L. Taylor

Case Western Reserve University, Cleveland, Ohio

January 8, 1981

Some Artifacts and Experimental Constraints in SANS Studies of Polymers

George D. Wignal1

Oak Ridge National Laboratory, Oak Ridge, Tennessee

January 26, 1981

Structural Aspects of Segmented Polyurethanes and Correlations With Mechanical Behavior Garth Wilkes

Virginia Polytechnic Institute and State University, Blacksburg, Virginia

January 29, 1981

Miscible Polymer Blends

Donald Paul

University of Texas, Austin, Texas

February 6, 1981

The Role of Mechanical Stress in Polymer Networks in Equilibrium and in Transport Alexander Silberberg

Weizmann Institute of Science, Rehovot, Israel

February 26, 1981

Block Copolymers as Surfactants Between Immiscible Solvents

Robert Cantor

College de France, Paris, France

March 3, 1981

Global and Local Structure Function in a Self-Repelling Chain Polymer

Thomas Witten

University of Michigan, Ann Arbor, Michigan

March 5, 1981

Composite Restorative Materials

Robert Denyer

Imperial Chemical Industries, Ltd., Macclesfield, England

March 23, 1981

Viscosity of Semi-Dilute Polymer Solutions

Mireille Adam

CEN de Saclay, Gif-sur-Yvette, France

March 25, 1981

New Aspects of the Microhardness of Polyethylene Relating to Texture and Morphology Francisco Balta Calleja

Instituto de Estructura de la Materia, Serrano, Spain

March 26, 1981

Phase Transition and Morphology in PVDF

B. Servet

Thomson-CSF, Orsay, France

March 26, 1981

Molecular Orientation and Chain Relaxation in Polystyrene Stretched Above $\mathrm{Tg}$ Lucien Monnerie

Ecole Superieure de Physique de Chimie Industrielles de Paris, Paris, France

March 27, 1981

High Pressure Phase Equilibria of Polyethylene/Ethylene Mixtures

Dominic Richon

Ecole Nationale Superieure des Mines, Paris, France

March 27, 1981

The Morphology of Polyethylene Crystallized Under the Influence of Flow David Grubb

Cornel1 University, Ithaca, New York

March 30, 1981 
Fundamental Processes in the Photo-0xidation of Polyolefins

James Guillet

Lash Miller Chemical Laboratories, Toronto, Canada

March 30, 1981

The Structure of Aromatic Polyesters

Ivan H. Hall

Purdue University, West Lafayette, Indiana

March 30, 1981

The Diffusion of Linear, Branched, and Cyclic Polymers in Concentrated Solutions and Melts of a

Linear Polymer

Jacob Klein

University of Cambridge, Cambridge, England

Apri1 3, 1981

Orientational Distribution and Molecular Motion in Oriented Deuterated Polyethylene From ${ }^{2} \mathrm{H}-\mathrm{NMR}$

Experiments

Hans Sillescu

University of Mainz, Mainz, West Germany

Apri1 3, 1981

Recent Developments in Vibrational Spectroscopy of Polymethylene Molecules

Guiseppe Zerbi

Institute of Macromolecular Chemistry, Milan, Italy

Apri1 9, 1981

Phase Behavior of Polystyrene (I) Poly(2,6-Dimethyl-1,4-Phenylene 0xide) (II) and Their Brominated Derivatives

Roger P. Kambour

General Electric Company, Schenectady, New York

April 17, 1981

Morphological and Mechanical Studies of Oriented Block Copolymers by SAXS, SANS, and EM Georges Hadziioannou

University of Massachusetts, Amherst, Massachusetts

Apri1 22, 1981

NMR Spatial Mapping for Medical Applications and Nondestructive Evaluation Jerome I. Kaplan

Indiana University, Indianapolis, Indiana

Apri1 23, 1981

Ion Distribution Around a Polyelectrolyte by the Monte Carlo Method

Bruno H. Zimm

University of California, San Diego, California

April 29, 1981

Micromechanics, Microstructure, and Fracture of Crazes

Edward J. Kraemer

Cornel1 University, Ithaca, New York

May 1, 1981

Mechanical Properties of Polymer Liquid Crystals

Masao Horio

Kyoto University, Kyoto, Japan

May 15, 1981

The Physical Properties of Acrylic Blends

Robert Kusy

University of North Carolina, Chapel Hill, North Carolina

May 27, 1981

On the Optical and Mechanical Behavior of Viscoelastic Fluids in Shear

Bernard D. Coleman

Carnegie-Mellon University, Pittsburgh, Pennsylvania

June 3,1981 
Rheology of Rod-Like Liquid Crystal Polymers

Kurt Wissbrun

Celanese Research, Summit, New Jersey

June 8,1981

Crystal Structure of a "Simple" Polymer PVF

Jerome B. Lando

Case Western Reserve University, Cleveland, Ohio

June 9, 1981

Structure Determination of Crosspolymerized Poly $(1,11$-Dodecadiyne)

Jerome B. Lando

Case Western Reserve University, Cleveland, Ohio

June 9,1981

Light Scattering and Electro-0ptics of Biomembrane

Hyuk Yu

University of Wisconsin, Madison, Wisconsin

June 12, 1981

The Feasibility of Screen Film Combinations in Intra-Oral Dental Radiography

Tomohero Okano

National Institute for Dental Research, Bethesda, Maryland

June 16, 1981

Activities of the Australian Dental Standards Laboratory and Research on Bonding to Teeth Derrick Beech

Australian Dental Standards Laboratory, Victoria, Australia

June 17, 1981

Relaxation of Stresses in Crazes at Cracked Tips and Rate of Craze Extension

Elio Passaglia

National Bureau of Standards, Washington, D.C.

June 18, 1981

Motion of Spheres in Polymer Solutions

Carlos Tiu

Auburn University, Auburn, Alabama

July 6,1981

"Absolute" Cross-Link Density Determinations

Emory Menefee

U.S. Dept. of Agriculture, Berkeley, California

July 7,1981

Applications of Finite Element Modeling in Dentistry

Otto Widera

University of Illinois, Chicago, Illinois

July 7,1981

Behavior of Ferroelectric Ceramics and PVF 2 Polymers Under Shock Loading

Francois Bauer

Institut Franco-Allemand de Recherches de Saint-Louis, Saint-Louis, France

July 8,1981

Molecular Weight Distribution From Stretch Relaxation: A New Algorithm for Relaxation

Distribution

Emory Menefee

U.S. Dept. of Agriculture, Berkeley, California

July 8,1981

NMR Study of Dissolution of Highly Insoluble Polysaccharides, Cellulose and Chitin

Marc Vincendon

Centre D'Etudes Nucleaires de Grenoble, Grenoble, France

July 10, 1981

A Visit of Research Institutes and Universities in Four Chinese Cities and Sightseeing to Sites Less-Frequented by Tourists"

Shu-Sing Chang

National Bureau of Standards, Washington, D.C.

July 14, 1981 
Integral Equation Method for the Evaluation of the Entropy of Polymer Chains Confined to Very Narrow Cylinders-Application to the Intermediate Phase of Polyethylene

Richard Priest

Naval Research Laboratory, Washington, D.C.

July 24, 1981

The Lowest Excited Electronic State in Trans-Polyacetylene

Eugene A. Imhoff

Cornel1 University, Ithaca, New York

August 4, 1981

Structure and Morphology of Nascent Polyethylene Obtained by Ziegler-Natta Catalysts Antonio Munoz-Escalona

Ministerio de Sanidad y Asistencia Social, Caracas, Venezuela

September 14, 1981

\section{Technical and Professional Committee Participation and Leadership}

Academy of Operative Dentistry

N. W. Rupp

Councilor

American Academy of Gold Foil Operators

N. W. Rupp

Councilor

American Association of Dental Research/International Association of Dental Research G. M. Brauer

Session Chairman

American Association of Dental Research/International Association of Dental Research W. E. Brown

Member: Biological Mineralization Research Award Committee

Mineralized Tissue Group, Chairman

Mineralized Tissue Group, Councilor

American Association of Dental Research/International Association of Dental Research J. M. Cassel

Washington Section Delegate

American Association of Dental Research/International Association of Dental Research J. A. Tesk

Member: Dental Materials Group

Executive Committee

American Chemical Society

J. M. Antonucci

Member: Board of Managers

Calendar Editor for Capital Chemists

American Chemical Society

G. M. Brauer

Member: Meetings and Expositions Committee

Special Events Committee, Chairman

American College of Dentists

N. W. Rupp

Member: Award Committee re Robert J. Nelsen, Chairman

American Dental Association Acceptance Program

J. A. Tesk

Member: Porcelain Metal Systems

American Institute of Mining, Metallurgical, and Petroleum Engineers

R. M. Waterstrat

Member: Committee on Alloy Phases

American Institute of Physics

R. K. Eby

Chapter Editor, 1981 Physics Handbook 
American Institute of Physics

E. A. Kearsley

Member: Committee for Public Policy

American National Standards Institute/MD156, Dental Materials and Devices

R. L. Bowen

Member: Toxicity Tests, Secretary

American National Standards Institute/MD156, Dental Materials and Devices G. M. Brauer

Member: Denture Resins, Chairman

American National Standards Institute/MD156, Dental Materials and Devices J. M. Cassel

Member: C054, Medical Devices Standards Management Board, Executive Committee

American National Standards Institute/MD156, Dental Materials and Devices N. W. Rupp

Member: Alloy for Dental Amalgams, Chairman

Amalgamators for Dental Amalgams, Chairman

Dispensers for Mercury and Alloys for Dental Amalgams, Chairman

Mercury for Amalgams, Chairman

Pit and Fissure Sealants

Restorative Materials Group, Chairman

American National Standards Institute/MD156, Dental Materials and Devices

J. A. Tesk

Member: Base Metal Alloys

Dental Implants, Secretary

Dental Materials, Instruments, and Equipment, Chairman

Porcelain/Alloy Systems

USA TAG for ISO TC106, Chairman

American Physical Society/Division of High Polymer Physics

F. A. Khoury

Member: Executive Committee

Committee on History of DMPP

American Physical Society/Division of High Polymer Physics

I. C. Sanchez

Member: Executive Committee

ASTM/Committee on Research and Technical Planning

E. Passaglia

Member: Subcommittee on Research, Chairman

ASTM D-2 Petroleum Products and Lubricants

L. J. Zapas

Member: SC.07 Flow Properties

SC.07A Newtonian Viscometry

ASTM D-9 Electrical Insulating Materials

W. P. Harris

Member: SC.05 Ceramic Products

SC. 12 Electrical Tests

SC. 16 Hookup Wire Insulation

SC. 17 Thermal Capabilities

SC. 90 Executive Committee

SC. 93 Education, Research, and Symposia

SC. 94 Nomenclature, Significance, and Statistics

SC. 95 Liaison

ASTM D-11 Rubber and Rubber-Like Materials

E. A. Kearsley

Member

ASTM D-11 Rubber and Rubber-Like Materials

G. B. McKenna

Member 
ASTM D-20 Plastics

R. K. Eby, Chairman-Elect

Member: SC.01 Executive Subcommittee

ASTM D-20 Plastics

J. D. Barnes

Member: $\quad$ SC.12 01efin Plastics

SC.13 Statistics

SC. 19 Sheeting

SC.70 Analytical Methods

WG. 07 Gas Permeability

ASTM D-20 Plastics

H. L. Wagner

Member: SC.70 Analytical Methods

WG.04 Liquid Size Exclusion Chromatography

WG.05 Molecular Weight Parameters, Chairman

ASTM D-27 Electrical Insulating Liquids and Gases

W. P. Harris

Member

ASTM E-9 Fatigue

G. B. McKenna

Member

ASTM E-9 Fatigue

R. W. Penn

Member

ASTM E-37 Thermal Measurements

S. S. Chang

Member: SC.01 Test Methods and Recommended Practices SC. 05 Thermophysical Properties

ASTM E-37 Thermal Measurements

J. H. Flynn

Member: SC.03 Nomenclature and Definitions, Chairman SC.90 Executive Subcommittee

ASTM E-45 Geothermal Energy and Resources

E. A. Kearsley

Hember: SC.02 Materials

ASTM F-2 Flexible Barrier Materials

J. D. Barnes

Member: SC.30 Test Methods, Chairman

SC. 93 Statistical Matters, Chairman

ASTM F-4 Medical and Surgical Materials and Devices

G. M. Brauer

Member: SC.20 Resources

WG.01 Polymeric Materials, Chairman

ASTM F-4 Medical and Surgical Materials and Devices J. M. Cassel

Member: SC.20 Resources

ASTM F-4 Medical and Surgical Materials and Devices R. K. Eby

Member

ASTM F-4 Medical and Surgical Materials and Devices G. B. McKenna

Member

Biomaterials (Journal)

G. C. Paffenbarger

Editorial Consultant 
Biomaterials Society

J. A. Tesk

Member: Finance Committee

Biomedical Research Support Grant

R. L. Bowen

Member: 1980-81 Review Committee

Case Western Reserve University Materials Research Laboratory Advisory Board

R. K. Eby

Member

Chemical Society of Washington

G. M. Brauer

Chairman, Membership Activities

Eleventh NATAS Conference

J. H. Flynn

Symposium on Kinetics of Chemical and Physical Processes, Chairman

Ferroelectrics Journal

M. G. Broadhurst

Guest Editor of Special Issue on PVDF

Fifth International Meeting on Ferroelectrics

M. G. Broadhurst

Member: U.S. Committee

Polymer Session Chairman

Geis Foundation for the Advancement of Dentistry

G. C. Paffenbarger

President

Member: Award Committee for Dental Editorial

Award Committee for Oral Surgery

Award Committee for Poreodontology

Government Interagency Panel on Adhesive Bonding Research

D. L. Hunston

Member

International Confederation for Thermal Analysis

J. H. Flynn

Member: Nomenclature Committee

Working Party on Symbols

International Council on Alloy Phase Diagrams

R. M. Waterstrat

Member

International Electrotechnical Commission/TC046, Cables, Wires and Waveguides for

Telecommunication Equipment

L. J. Zapas

Member: WG.03 Measuring Methods of Polyethylene

International Standards Organization/TC106, Dental Materials and Devices

G. M. Brauer

Member: Task Force on Color Stability

International Standards Organization/TC106, Dental Materials and Devices N. W. Rupp

Member: Restorative Materials

WG. 01 U.S. Delegation, Chairman

TG7 Alloys for Dental Amalgams, Chairman

WG.06 Amalgamation Devices and Accessories, Chairman

International Standards Organization/TC106, Dental Materials and Devices J. A. Tesk

USA TAG, Chairman 
International Standards Organizations/TC150, USTAG for Implants for Surgery

J. M. Cassel

Chairman

International Standards Organization/TC150, USTAG for Cardiovascular Implants

J. M. Cassel

NBS Representative

National Bureau of Standards Advisory Panel for Tariffs on Electron Microscopes Under Florence Agreement

F. A. Khoury

Member

National Center for Small Angle Scattering

C. C. Han

Member: Users Committee

National Institute of Dental Research

R. L. Bowen

Member: Long Range Research Planning and State-of-the-Science on Composites, Consultant Panel on Restorative Materials Program

Review Committee for ADA

National Research Council Panel on Polymer Science and Engineering

R. K. Eby

Member

North American Thermal Analysis Society

J. H. Flynn

Editor: NATAS Notes

Member: Awards Committee

Executive Committee

Publications Committee, Chairman

Ohio Dental Association

G. C. Paffenbarger

Member: Callahan Memorial Committee

Polymer Degradation and Stability (Journal)

J. H. Flynn

Editorial Board Member

Society of Plastics Engineers

R. K. Eby

Plember: Selection Committee for SPE International Award for Research, Chairman

Society of Plastics Engineers

G. B. McKenna

Member: Technical Publications Committee

Society of Rheology

D. L. Hunston

Member: Committee on Education, Chairman

Society of Rheology

E. A. Kearsley

Member: Executive Committee

Society of Rheology

G. B. McKenna

Member: Committee on Education

Local Arrangements 1981 Spring Meeting, Chairman

U.S. Air Force

N. W. Rupp

National Civilian Consultant to the Surgeon General

University of Massachusetts Advisory Board of Polymer Research Institute

R. K. Eby

Member 
University of Virginia, Department of Materials Science

G. C. Paffenbarger

Consultant

Washington Area MINC Users Group

J. D. Barnes

Chairman

\section{Publications}

Interpretation of Dynamic Scattering From Polymer Solutions

A. Z. Akcasu, M. Benmouna, and C. C. Han

Polymer, 21, 866 (1980)

The Accelerative and Adhesive Bonding Capabilities of Surface-Active Accelerators

J. M. Antonucci, D. N. Misra, and R. J. Peckoo

J. Dent. Res. (in press)

New Monomers for Use in Dentistry

J. M. Antonucci

Biomedical and Dental Applications of Polymers, C. G. Gebelein and F. F. Koblitz, Eds., P. 357

(1981)

Slow-Acting Amine Polymerization Accelerators. Para-Dimethylaminobenzoic Acid and Its Ethyl Ester

J. M. Antonucci, R. J. Peckoo, C. Schruhl, and E. E. Toth

J. Dent. Res., 60, 1325 (1981)

An Amine Accelerator for Colorfree Curing of Cold-Curing Methyl Methacrylate Denture Systems

H. Argentar, J. A. Tesk, and E. E. Parry

J. Amer. Dent. Assoc., 102, 664 (1981)

A Theory of the Effects of Pressure on Finite Elastic Shear

B. Bernstein and E. A. Kearsley

Rheology, G. Arkarita, G. Marrucci, and L. Nicholais, Eds., p. 305 (1980)

Stability and Cold Drawing of Viscoelastic Bars

B. Bernstein and L. J. Zapas

J. Rheology, 25, 83 (1981)

Viscoelastic Fracture of Structural Adhesives

J. L. Bitner, J. L. Rushford, W. S. Rose, D. L. Hunston, and C. K. Riew

J. Adhesion (in press)

Adhesive Bonding of Various Materials to Hard Tooth Tissues. XXIII. Isotonic Monomer Formulations

R. L. Bowen

J. Dent. Res., 60, 901 (1981)

Adhesive Bonding of Various Materials to Hard Tooth Tissues. XXIV. Recent Developments in Adhesive Resin Formulations for Dentin

R. L. Bowen

Proceedings of International Congress of Implantology and Biomaterials in Stomatology, p. 66 (1980)

Initiator-Accelerator Systems for Acrylic Resins and Composites

G. M. Brauer

Biomedical and Dental Applications of Polymers, C. G. Gebelein and F. F. Koblitz, Eds., p. 395 (1981)

Marginal Adaptation of BIS-GMA Based Composites Containing Various Diluents

G. M. Brauer, D. M. Dulik, H. N. Hughes, K. Dermann, and N. W. Rupp

J. Dent. Res. (in press)

4-N,N-Dialkylaminophenylethanols, Alkanoic Acids, and Esters: New Accelerators for Dental Composites

G. M. Brauer, J. W. Stansbury, and J. M. Antonucci

J. Dent. Res. (in press) 
High Polymer Physics

M. G. Broadhurst, S. S. Chang, G. T. Davis, E. A. DiMarzio, R. K. Eby, B. M. Fanconi,

J. H. Flynn, C. C. Han, F. A. Khoury, H. Markovitz, J. E. McKinney, F. I. Mopsik, E. Passaglia, I. C. Sanchez, L. E. Smith, D. L. VanderHart, and L. J. Zapas

AIP 50th Anniversary Handbook (in press)

Ferroelectric Polarization in Polymers

M. G. Broadhurst and G. T. Davis

Ferroelectrics, 32, 177 (1981)

Dielectric Properties of PVDF--Low Field and High Field Phenomena and Time Effects

M. G. Broadhurst, G. T. Davis, and A. S. DeReggi

Proceedings of the Symposium on High Energy Density Capacitors and Materials (in press)

Pyroelectricity and Charge Transport in a Copolymer of Vinylidene Fluoride and

Tetrafluoroethylene

M. G. Broadhurst, G. T. Davis, A. S. DeReggi, S. C. Roth, and R. E. Collins

Polymer (in press)

Summary of Workshop on Morphology of Polyethylene Power Cable Insulation

M. G. Broadhurst, F. A. Khoury, A. J. Bur, G. T. Davis, and W. P. Harris

Electric Power Research Institute Report (in press)

Guest Editorial on Piezoelectric Polymers

M. G. Broadhurst, F. Micheron, and Y. Wada

Ferroelectrics (in press)

Hydrolytic Degradation of Polyester Polyurethane Foams

D. W. Brown, R. E. Lowry, and L. E. Smith

ACS Polymer Preprints, 22, 223 (1981)

Kinetics of Reaction Between Polyester Acid and Carbodiimides in Dry Polyester Diols and in a Polyester Polyurethane

D. W. Brown, R. E. Lowry, and L. E. Smith

Macromolecules, 14, 659 (1981)

Thermodynamics of Apatite Crystal Growth and Dissolution

W. E. Brown and L. C. Chow

J. Crystal Growth, 53,31 (1981)

Crystal Chemistry of Octacalcium Phosphate

W. E. Brown, M. Mathew, and M. S. Tung

Prog. Crystal Growth, Charact., 4, 59 (1981)

Role of Octacalcium Phosphate in the Incorporation of Impurities Into Apatites

W. E. Brown, M. S. Tung, and L. C. Chow

Proceedings of the Second International Congress on Phosphorus Compounds, p. 59 (1981)

Resistivity and Piezoelectric Measurements on Oriented and Unoriented Thick PVF 2 Films

A. J. Bur

Polymer (in press)

Depolarization of Poled Polyvinylidene Fluoride Samples With "Thick" Electrodes

A. J. Bur and A. K. Tsao

Ferroelectrics (in press)

Properties and Interactions of Oral Structures and Restorative Materials

J. M. Cassel, J. A. Tesk, G. M. Brauer, J. M. Antonucci, W. Wu, J. E. Mckinney, R. P. Whitlock, and R. Hinman

NBSIR $80-2168(1980)$

\section{A Mechanism for Incorporation of Carbonate Into Apatite \\ N. S. Chickerur, M. S. Tung, and W. E. Brown \\ Calcif. Tissue Int., 32,55 (1980)}

Reactions of Powdered Human Enamel and Fluoride Solutions With and Without Intermediate $\mathrm{CaHPO}_{4} \cdot 2 \mathrm{H}_{2} \mathrm{O}$ Formation

L. C. Chow, M. K. Guo, C. C. Hsieh, and Y. C. Hong

J. Dent. Res., 59, 1447 (1980) 
Diffraction From Nonperiodic Structures: The Molecular Conformation of Polytetrafluoroethylene (Phase II)

E. S. Clark, J. J. Weeks, and R. K. Eby

ACS Symposium Series 141, "Diffraction Methods for Structural Determination of Fibrous Polymers, p. $183(1980)$

Transport Properties of Annealed Drawn Low Density Polyethylene (LDPE)

F. DeCandia, R. Russo, V. Vittoria, and A. Peterlin

J. Polym. Sci., Polym. Phys. Ed., 18, 2083 (1980)

Evaluation of Methods of Characterizing the Porosity of Porous Polymeric Implant Materials: A Review of the Current Status of Porosity Measurements

R. E. Deh 1, W. H. Grant, and J. M. Cassel

NBSIR 81-2212 (February 1981)

Forward Depolarized Scattering of Semi-Dilute Solutions of Poly ( $\alpha$-Methyl Styrene)

M. Delsanti, C. C. Han, and H. Yu

ACS Polymer Preprints, 22, 76 (1981)

Piezoelectric Polymer Probe for UTtrasonic Applications

A. S. DeReggi, S. C. Roth, J. M. Kenney, and S. Edelman

J. Acoust. Soc. Am., 69, 853 (1981)

Free Radicals and New End Groups Resulting From Chain Scission. I. r-Irradiation of Polyethylene

K. L. DeVries, R. H. Smith, and B. M. Fanconi

Polymer, 21, 949 (1980)

Factor-Jump Thermogravimetry Applied to Polymer Degradations

B. Dickens

ACS Organic Coatings \& Plastics Chemistry, 44, 670 (1981)

Factor-Jump Thermogravimetry Applied to Polymer Degradations

B. Dickens

ACS Polymer Preprints, 22, 316 (1981)

Therma? Degradation and Oxidation of Polystyrene Studied by Factor-Jump Thermogravimetry

B. Dickens

Polym. Deg. \& Stab., 2, 249 (1980)

Investigation of Epitaxy Relationships Between $\mathrm{Ca}_{5}\left(\mathrm{PO}_{4}\right)_{3} \mathrm{OH}$ and Other Calcium Ortho-Phosphates

B. Dickens and L. W. Schroeder

J. Res. Nat. Bur. Stand., 85, 347 (1980)

Effect of Diluent Monomer on the Physical Properties of BIS-GMA-Based Composites

D. Dulik, R. Bernier, and G. M. Brauer

J. Dent. Res., 60,983 (1981)

Observations by High Resolution ${ }^{13} \mathrm{C}$ NMR of Cellulose I Related to Morphology and Crystal Structure W. L. Earl and D. L. VanderHart

Macromolecules (in press)

1980 Annual Report of the Polymer Science and Standards Division

R. K. Eby, L. E. Smith, M. G. Broadhurst, B. M. Fanconi, I. C. Sanchez, and J. M. Cassel NBSIR 81-2263 (Apri1 1981)

Crystalline Polymers

R. K. Eby, A. Peterlin, F. A. Khoury, M. G. Broadhurst, and B. M. Fanconi

State of Polymer Science in the U.S., National Academy of Sciences (in press)

Free Radicals and New End Groups Resulting From Chain Scission. II. Mechanical Degradation of Polyethylene

B. M. Fanconi, J. P. Colson, K. L. DeVries, and R. H. Smith

Polymer (in press)

Energy Calculations of the Crystal Structure of the Low Temperature Phase (II) of Polytetra-

fluoroethylene

B. L. Farmer and R. K. Eby

Polymer (in press) 
A Collection of Kinetic Data for the Diffusion of Organic Compounds in Polyolefins J. H. Flynn

Polymer (in press)

Analysis of Thermogravimetric Kinetics--Overcoming Complications of Thermal History J. H. Flynn

Thermal Analysis in Polymer Characterization (in press)

Role of Thermal Analysis in the Lifetime Prediction of Polymers

J. H. Flynn

Proceedings of the Second European Symposium on Thermal Analysis (in press)

Thermogravimetric Analysis Kinetics

J. H. Flynn

ACS Organic Coatings \& Plastics Chemistry, 44, 657 (1981)

Thermogravimetric Analysis Kinetics

J. H. Flynn

ACS Polymer Preprints, 22, 310 (1981)

${ }^{13} \mathrm{C}$ NMR in Organic Solids: Limits to Spectral Resolution and to Determination of Molecular Motion

A. N. Garroway, D. L. VanderHart, and W. L. Earl

Philos. Trans. R. Soc. London, A299, 609 (1981)

Adhesive Bonding of Various Materials to Hard Tooth Tissues: Tracer Study of Mordant Adsorption on Ename 1

T. E. Gills and R. L. Bowen

Orthodontics: The State of the Art, H. G. Barrer, Ed., p. 163 (1981)

The Adsorption of Human Albumin and $\gamma$-Globulin on Hydrophobic and Hydrophilic Surfaces W. H. Grant and R. E. Deh1

Adhesion and Absorption of Polymers, Part B, L. H. Lee, Ed., p. 827 (1980)

A Monte-Carlo Calculation of the Hydrodynamic Radius at the Theta Point-Deviations From Analytical Gaussian Behavior

C. M. Guttman, F. L. McCrackin, and C. C. Han

Macromolecules (in press)

Concentration Dependence of Diffusion Coefficient of Various Molecular Weights and Temperatures C. C. Han and A. Z. Akcasu

Polymer (in press)

Dynamic Light Scattering of Dilute Polymer Solutions in the Non-Asymptotic q-Region

C. C. Han and A. Z. Akcasu

Macromolecules (in press)

Dynamic Light Scattering of Polymer Solutions in the Intermediate Momentum Transfer Region C. C. Han and A. Z. Akcasu

ACS Polymer Preprints, 22, 68 (1981)

On the Dynamic Structure Factor, $S(q, \omega)$ of Polymers in Dilute Solution

C. C. Han and A. Z. Akcasu

Polymer (in press)

An Experimental Study of Flow-Generated Waves on an Elastic Surface

R. J. Hansen, D. L. Hunston, C. C. Ni, and M. M. Reischman

J. Sound Vibration, 68,317 (1980)

Hydrodynamic Drag and Surface Deformations Generated by Liquid Flows Over Flexible Surfaces R. J. Hansen, D. L. Hunston, C. C. Ni, M. M. Reischman, and J. W. Hoyt

Viscous Flow Drag Reduction, H. Hough, Ed., p. 439 (1980)

Viscoelastic Characterization of Structural Adhesives Via Forced Oscillation Experiments D. L. Hunston, W. D. Bascom, E. E. Wells, J. D. Fahey, and J. L. Bitner Proceedings of the International Conference on Adhesion and Adsorption of Polymers (1980)

Fracture of Rubber-Toughened Adhesives: Loading Rate and Temperature D. L. Hunston, J. L. Bitner, J. L. Rushford, J. Oroshnik, and W. S. Rose Proceedings of the California Conference on Rubber-Toughened Plastics, I, 1 
Mechanical Properties of Solid Polymers as Modeled by a Simple Epoxy

D. L. Hunston, W. Carter, and J. L. Rushford

Developments in Adhesives, A. J. Kinlock, Ed. (1981)

Failure in Elastomer-Toughened Epoxies: Viscoelastic Effects

D. L. Hunston, J. L. Rushford, J. L. Bitner, J. Oroshnik, and R. S. Rose

J. Elastomers and Plastics, 12, 133 (1980)

Effect of Molecular Parameters on the Flow Rate Dependence of Drag Reduction and Similar Phenomena D. L. Hunston and J. L. Zakin

Viscous Flow Drag Reduction, H. Hough, Ed., p. 337 (1980)

Mechanical Degradation in Dilute Polystyrene Solutions

D. L. Hunston and J. L. Zakin

Polym. Eng. Sci., 20, 517 (1980)

The Role of Zinc in Dental Amalgams

L. B. Johnson and G. C. Paffenbarger

J. Dent. Res., 59, 1412 (1980)

Crystal Structure of Tritin (II) Hydroxide Oxide Phosphate, $\mathrm{Sn}_{3} \mathrm{O}(\mathrm{OH}) \mathrm{PO}_{4}$

T. H. Jordan, B. Dickens, L. W. Schroeder, and W. E. Brown

Inorg. Chem., 19, 2551 (1980)

A Basis for Predicting Long-Time Behavior From Short-Time Tests of Geothermal Sealants

E. A. Kearsley

Geothermal Seals, ASTM (in press)

Determining an Elastic Strain-Energy Function From Torsion and Simple Extension

E. A. Kearsley

J. Appl. Phys., 51, 4541 (1980)

Some Methods of Measurement of an Elastic Strain-Energy Function of the Valanis-Landel Type

E. A. Kearsley and L. J. Zapas

J. Rheology, 24, 483 (1980)

Deformation and Failure of Ultra High Molecular Weight Polyethylene

F. A. Khoury

Soc. Plastics Eng., 27, 82 (1981)

Relationship Between Morphology and Mechanical Properties of Ultra High Molecular Weight

Polyethylene

F. A. Khoury

NBSIR 81-2209 (Apri1 1981)

Monte Carlo Studies of Polymer Chain Dynamics: Lattice Dependence

D. E. Kranbuehl and P. H. Verdier

ACS Polymer Preprints, 21, 195 (1980)

Effects of Poling Field and Time on Pyroelectric Coefficient and Polarization Uniformity in Polyvinyl Fluoride

S. B. Lang, A. S. DeReggi, and M. G. Broadhurst

Ferroelectrics (in press)

Lead Alkali Apatites Without Hexad Anion: The Crystal Structure of $\mathrm{Pb}_{8} \mathrm{~K}_{2}\left(\mathrm{PO}_{4}\right)_{6}$

M. Mathew, W. E. Brown, M. Austin, and T. Negas

J. Solid State Chem., 35, 69 (1980)

New Struvite Analogue, $\mathrm{MgNaPO}_{4} \cdot 7 \mathrm{H}_{2} \mathrm{O}$

M. Mathew, P. Kingsbury, S. Takagi, and W. E. Brown

Acta Cryst. (in press)

The Crystal Structure of Synthetic Chukhrovite, $\mathrm{Ca}_{4} \mathrm{AlSi}\left(\mathrm{SO}_{4}\right) \mathrm{F}_{13} \cdot 12 \mathrm{H}_{2} \mathrm{O}$

M. Mathew, S. Takagi, K. R. Waerstad, and A. W. Frazier

Amer. Minera1., 66, 392 (1981)

Configurational Properties of Comb-Branched Polymers

F. L. McCrackin and J. Mazur

Macromolecules (in press) 
Mechanical Properties of Some Composite Biomaterials After Implantation as Bone Plates

G. B. McKenna, G. W. Bradley, H. K. Dunn, and W. 0. Statton

Biomaterials, 1,189 (1980)

Deformation and Failure of Ultra High Molecular Weight Polyethylene

G. B. McKenna, J. M. Crissman, and F. A. Khoury

Soc. Plastic Eng., 27, 84 (1981)

Time-Dependent Failure of a Polyolefin Rubber Candidate Material for Blood Pump Applications

G. B. McKenna and R. W. Penn

J. Biomed. Matls. Res., 14, 689 (1980)

Cyclic Loading of Carbon Black Filled Butyl Rubber

G. B. McKenna and L. J. Zapas

Rubber Chem. Technol. (in press)

The Normal Stress Response in Non-Linear Viscoelastic Materials--Some Experimental Findings

G. B. McKenna and L. J. Zapas

J. Rheology, 24, 367 (1980)

Viscoelastic Behavior of Poly(Methyl Methacrylate). Prediction of Extensional Response From Torsional Data

G. B. McKenna and L. J. Zapas

Rheology, G. Astarita, G. Marrucci, and L. Nicolais, Eds., p. 299 (1980)

Relationship Between Subsurface Damange and Wear of Dental Restorative Composites

J. E. Mckinney and W. Wu

J. Dent. Res., 60,583 (1981)

Monomolecular Adsorption Isotherms

D. N. Misra

J. Coll. Inter. Sci., 77, 543 (1980)

Adsorption of $\mathrm{N}$-(2-Hydroxy-3-Methacryloxypropyl)-N-Phenylglycine (NPG-GMA) on Cupric Ion-Enriched Hydroxyapatite Surface to Improve Chemical Bonding Between Dental Resins and Teeth

D. N. Misra and R. L. Bowen

Biomaterials, 2, 78 (1981)

Adsorptive Bonding to Hydroxyapatite. I. Adsorption of Anthranilic Acid--The Effect of Solvents.

Search for Surface Bonding Groups for Coupling Agents to Teeth

D. N. Mịsra and R. L. Bowen

Biomaterials, 2, 28 (1981)

Adsorption of a Polyfunctional Surface-Active Amine Accelerator on Hydroxyapatite

D. N. Misra, R. L. Bowen, J. M. Antonucci, and W. F. Cuthrel1

J. Coll. Inter. Sci., 77, 143 (1980)

The Numerical Analysis of the Thermal Pulse Experiment

F. I. Mopsik and A. S. DeReggi

Proceedings of the Conference on Electrical Insulation and Dielectric Phenomenon, p. 251 (1980)

Chain Folding in Lamellar Crystals

A. Peterlin

Macromolecules, 13, 777 (1980)

Molecular Interpretation of the Load-Elongation Curve of Crystalline Polymers

A. Peterlin

Intern. J. Polym. Mater., 8, 285 (1980)

Empirical Relation for Diffusion of Gases in Hydrocarbon Polymers: Interpretation in Terms of

Fractional Free Volume

A. Peterlin and F. L. McCrackin

J. Polym. Sci., Polym. Phys. Ed., 19, 1003 (1981)

Apparatus for Infrared Measurement of Sorption/Desorption in Strained Polymeric Films

J. C. Phillips, A. Peterlin, and P. F. Waters

J. Polym. Sci., Polym. Phys. Ed., 19, 789 (1981)

Interfacial Tension Theory of Low and High Molecualr Weight Liquid Mixtures

C. I. Poser and I. C. Sanchez

Macromolecules, 14, 361 (1981) 
Energy Flow and Thermal Conductivity in One-Dimensional, Harmonic, Isotopically Disordered Crystals R. J. Rubin

Perspectives in Statistical Physics, H. J. Ravache, Ed., p. 113 (1981)

Radon Transport Through and Exhalation From Building Materials, a Review and Assessment

R. J. Rubin, R. Colle, L. I. Knab, and J. M. R. Hutchinson

NBS Technical Note 1139 (October 1980)

Random Walks on Lattices. The Problem of Visits to a Set of Points Revisited

R. J. Rubin and G. H. Weiss

J. Math. Phys. (in press)

Statistical Thermodynamics of Bulk and Surface Properties of Polymer Mixtures

I. C. Sanchez

J. Macromol. Sci.-Phys., B17, 565 (1980)

Migration Models for Polymer Additives

I. C. Sanchez, S. S. Chang, and L. E. Smith

Polymer News, 6, 249 (1980)

Cell Model Theory of Polymer Solutions

I. C. Sanchez and D. J. Lohse

Macromolecules, 14, 131 (1981)

Application of Photon Correlation Spectroscopy

D. Schaefer and C. C. Han

Polymer Solutions (in press)

Polymer Dynamics: When Do Scaling Laws Apply?

D. W. Schaefer and C. C. Han

ACS Polymer Preprints, 22, 66 (1981)

Raman Spectrum From a Single Crystal of $\mathrm{SnHPO}_{4}$

L. W. Schroeder, T. H. Jordan, and W. E. Brown

Spectrochimica Acta, 37A, 21 (1981)

Characterizing the Interfiber Bonding of Currency Paper Pulps

J. C. Smith and E. E. Toth

NBSIR 80-2190 (1980)

Models for the Migration of Low Molecular Weight Additives in Polyolefins

L. E. Smith, S. S. Chang, G. A. Senich, F. L. McCrackin, and F. W. Wang

NBSIR 81-2264 (Apri1 1981)

Low-Frequency Raman Study of Drawn Polyethylene

R. G. Snyder, J. R. Scherer, and A. Peterlin

Macromolecules, 14, 77 (1981)

Water Rich Hydrates. The Crystal Structure of $\mathrm{Mg}_{2} \mathrm{KH}\left(\mathrm{AsO}_{4}\right)_{2} \cdot 15 \mathrm{H}_{2} \mathrm{O}$ and $\mathrm{Mg}_{2} \mathrm{KH}\left(\mathrm{PO}_{4}\right)_{2} \cdot 15 \mathrm{H}_{2} \mathrm{O}$

S. Takagi, W. E. Brown, and M. Mathew

Acta Cryst. (in press)

The Structure of Diammonium Tricalcium Bis(Pyrophosphate) Hexahydrate

S. Takagi, M. Mathew, and W. E. Brown

Acta Cryst., B36, 2526 (1980)

Physical Metallurgy of Dental Casting Alloys

J. A. Tesk and R. M. Waterstrat

Encyclopedia of Materials, Science, and Engineering (in press)

Consideration of Some Factors Influencing Compatibility of Dental Porcelains and Alloys, Part II:

Porcelain/Alloy Stress

J. A. Tesk, R. P. Whitlock, G. E. 0. Widera, A. Holmes, and E. E. Parry

Proceedings of the Fourth International Precious Metals Conference (1981)

Characterization of Ligand Binding Systems by Continuous Distributions of Arbitrary Shape

A. K. Thakur, P. J. Munson, D. L. Hunston, and D. Rodbark

Anal. Biochem., 103, 240 (1980) 
Influence of Molecular Packing on Solid-State ${ }^{13} \mathrm{C}$ Chemical Shifts: The $n$-Alkanes

D. L. VanderHart

J. Magn. Reson. (in press)

Resolution in ${ }^{13} \mathrm{C}$ NMR of Organic Solids Using High Power Proton Decoupling and Magic Angle Sample Spinning

D. L. VanderHart, W. L. Earl, and A. N. Garroway

J. Magn. Reson. (in press)

A Low Shear, High Temperature Rotational Viscometer: The Viscosity of Ultra High Molecular Weight Polyethylene

H. L. Wagner and J. G. Dillon

ACS Polymer Preprints, 22, 260 (1981)

Novel Cell for Light Scattering From Solutions

F. W. Wang and B. H. Zimm

Biopolymers, 20, 1333 (1981)

Analysis of Selected Alloys in the Systems $\mathrm{Cr}-\mathrm{Pd}, \mathrm{Cr}-\mathrm{Ru}, \mathrm{V}-\mathrm{Pd}$, and Ta-Pt

R. M. Waterstrat

J. Less-Common Metals (in press)

Binary Phase Diagrams of Transition Elements

R. M. Waterstrat

NBS Special Publication 564 (May 1980)

Structure Relationships Affecting the Stability of A15- and $\mathrm{Ti}_{3} \mathrm{P}$-Type Compounds

R. M. Waterstrat

J. Solid State Chem., 37,370 (1981)

The Crystal Structure of the Low Temperature Phase (II) of Polytetrafluoroethylene J. J. Weeks, E. S. Clark, and R. K. Eby

Polymer (in press)

Disorder in the Crystal Structures of Phases I and II of Copolymers of Tetrafluoroethylene and Hexafluoropropylene

J. J. Weeks, R. K. Eby, and E. S. Clark

Polymer (in press)

Consideration of Some Factors Influencing Compatibility of Dental Porcelains and Alloys, Part I: Thermo-Physical Properties

R. P. Whitlock, J. A. Tesk, G. E. 0. Widera, A. Holmes, and E. E. Parry

Proceedings of the Fourth International Precious Metals Conference (1981)

Molecular Interpretations of Modulus and Swelling Relations in Natural Rubber Cross-Linked by Dicumyl Peroxide

L. A. Wood

Rubber Chem. Technol., 53, 116 (1980)

Representation of Long-Time Creep in a Pure-Gum Rubber Vulcanizate

L. A. Wood

J. Res. Nat. Bur. Stand., 85, 283 (1980)

Representation of Long-Time Creep in a Pure-Gum Rubber Vulcanizate

L. A. Wood

Rubber Chem. Technol. (in press)

Application of Porod's Law to Fixed Particles With Preferred Orientation

W. Wu

J. Polym. Sci., Polym. Phys. Ed., 18, 1659 (1980)

The Correlation Function of Agglomerated Particles and Its Applications in Electromagnetic Scattering

W. Wu

J. Polym. Sci., Polym. Phys. Ed., 18, 1993 (1980)

Stretching of Nylon 66 Yarns Saturated With Dry $\mathrm{HCl}$

W. Wu and W. B. Black

ACS Polymer Preprints, 22, 248 (1981) 
Deformation of Doubly Oriented Polyethylene, Nylon 66, and Poly(Ethylene Terephthalate)

W. Wu, W. B. Black, and P. G. Simpson

Polymer, 21,992 (1980)

A Silver Staining Technique for Investigating Wear of Restorative Dental Composites

W. Wu and E. N. Cobb

J. Biomed. Mat1s. Res., 15, 343 (1981)

Influence of Chemicals on In Vitro Wear of Dental Restorative Composites

W. Wu and J. E. McKinney

J. Dent. Res., 60, 371 (1981)

Morphology and Tensile Property Relations in High-Strength/High-Modulus Polyethylene Fiber

W. Wu, P. J. Simpson, and W. B. Black

J. Polym. Sci., Polym. Phys. Ed., 18, 751 (1980)

Alkane Chain Conformation as a Function of Pressure

S. L. Wunder

High Pressure Science and Technology, B. Vodar and P. Marteau, Eds., p. 676 (1980)

Effect of Polymer Molecular Variables on Drag Reduction

J. L. Zakin and D. L. Hunston

J. Macromol. Sci. (in press)

\section{Patents}

Composite Dental Material

R. L. Bowen

July 29,1980

U.S. Patent $4,215,933$

Tertiary Aromatic Amine Accelerators in Acrylic Resin

H. Argentar

January 6, 1981

U.S. Patent $4,243,763$

Non-Polymerization Inhibiting, High Strength Dental Cements, Restoratives and Adhesive Based on Vanillic Acid Esters, Carboxylic Acids and Zinc Oxide

G. M. Brauer, J. W. Stansbury, and H. Argentar

Filed January 1981

Use of Polyfunctional Surface-Active Comonomers and Other Agents to Improve Adhesion Between a Resin or Composite Material and a Substrate

R. L. Bowen

February 17, 1981

U.S. Patent $4,251,565$

Arc-Furnace for the Production of Small Investment Castings

R. M. Waterstrat

Filed April 1981

Isotonic Monomer Formulation

R. L. Bowen

Filed May 1981

Polymeric Solid State Battery

C. K. Chiang

Filed May 1981

\section{Workshop}

Morphological Studies of Power Cable Insulation Failure

Electric Power Research Institute, Gaithersburg, Maryland M. G. Broadhurst

May 1981 


\section{External Recognition}

1981 Hollenback Memorial Prize

Academy of Operative Dentistry

February 1981

R. L. Bowen

1980 Mettler Award in Thermal Analysis North American Thermal Analysis Society October 1980

J. H. Flynn

Fellow

North American Thermal Analysis Society

October 1980

J. H. Flynn 


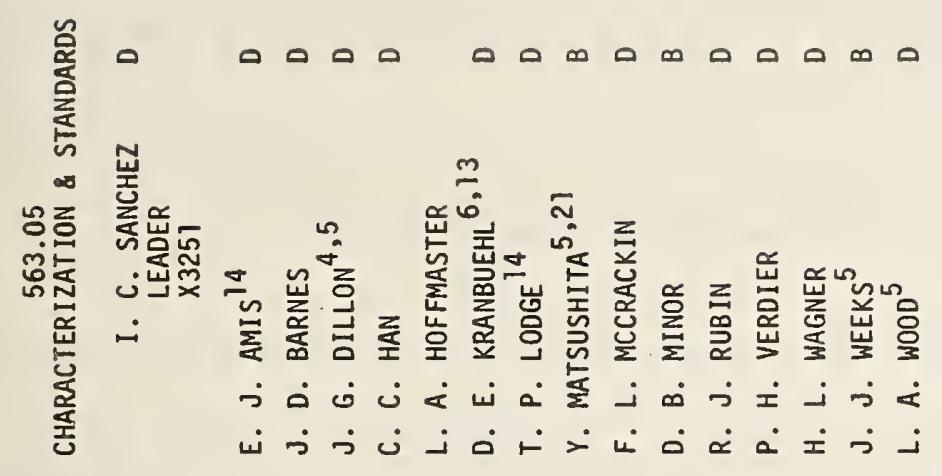

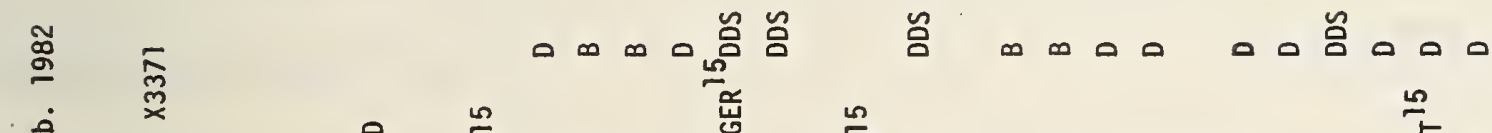 落
崖

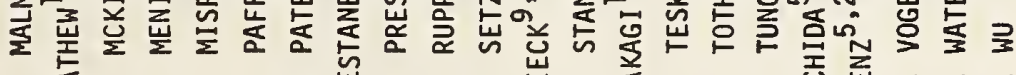

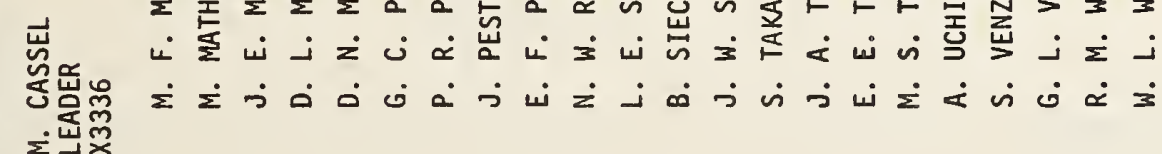

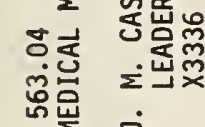
$\dot{j}$

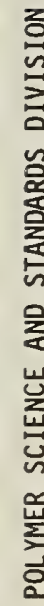

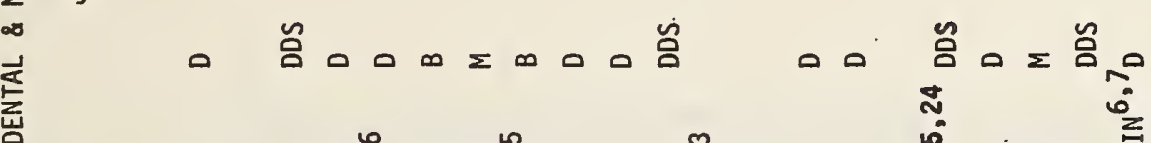

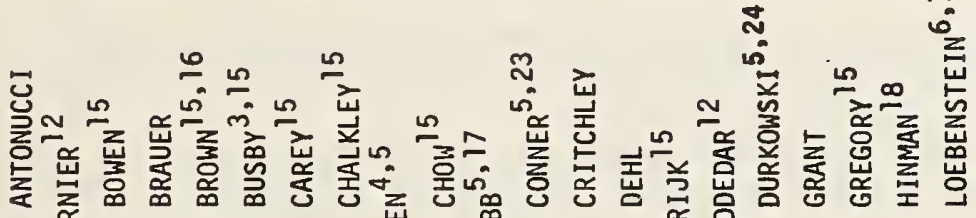

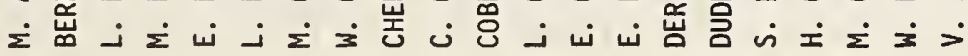

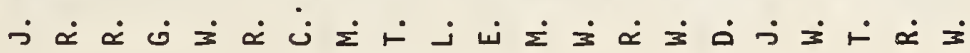

蒿㞱

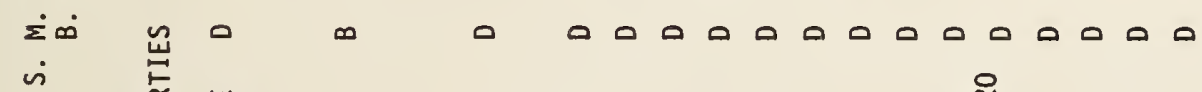

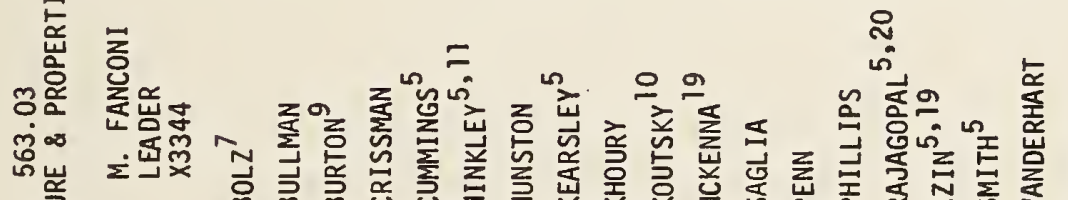

可

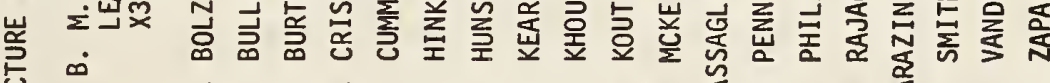

若总

点

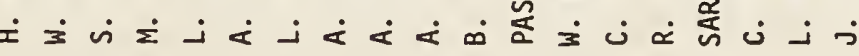

호ㅇㅝㅗㅇ

$\dot{j} \dot{0} \dot{j} \dot{j} \dot{j} \dot{0} \dot{L} \dot{j} \dot{0} \dot{\alpha} \dot{j} \dot{x} \dot{0} \dot{0} \dot{0}$

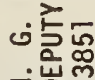

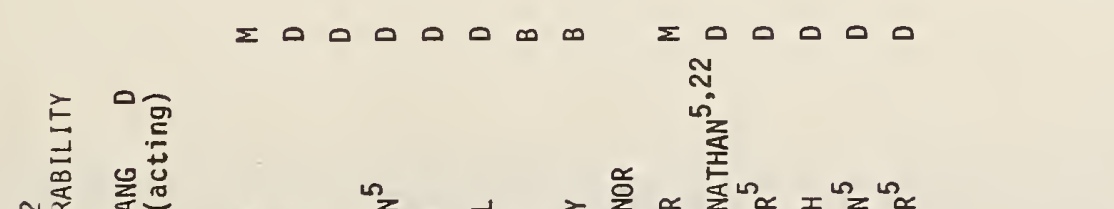

ก䓂

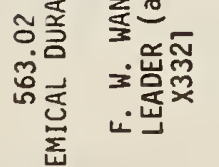

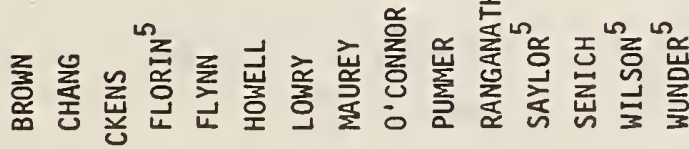

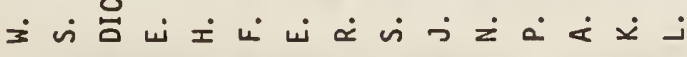

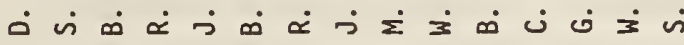

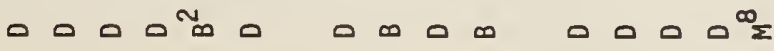

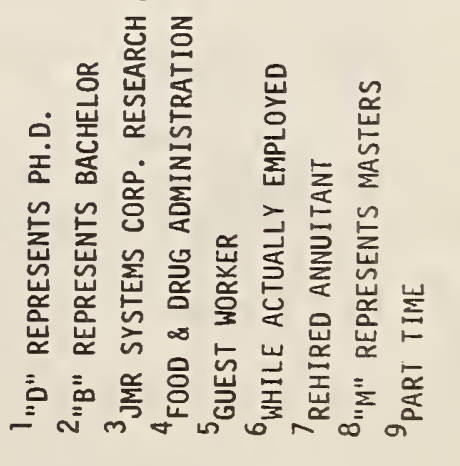


NBS-114A (REV. 2-8C)

\begin{tabular}{|c|c|c|}
\hline $\begin{array}{l}\text { U.S. DEPT. OF COMM. } \\
\text { BIBLIOGRAPHIC DATA } \\
\text { SHEET (See instructions) }\end{array}$ & $\begin{array}{l}\text { 1. PUBLICATION OR } \\
\text { REPORT NO. } \\
\text { NBSIR } 82-2452\end{array}$ & 2. Performing Organ. Report Noy $\begin{array}{r}\text { 3. Publication Date } \\
\text { March } 1982\end{array}$ \\
\hline
\end{tabular}

4. TITLE AND SUBTITLE

Polymer Science and Standards Division Annual Report 1981

5. AUTHOR(S)

R. K. Eby, M. G. Broadhurst, J. M. Cassel, B. M. Fanconi, I. C. Sanchez, L. E. Smith

6. PERFORMING ORGANIZATION (If joint or other than NBS, see in structions)

NATIONAL BUREAU OF STANDARDS

DEPARTMENT OF COMMERCE

WASHINGTON, D.C. 20234

9. SPONSORHAG ORGANHZATIOTH INAT IE AND COHALETE ADDRESS (Stfeet, City, Stote, ZIF)

7. Contract/Grant No.

8. Type of Report \& Period Covered

10. SUPPLEMENTARY NOTES

[.] Document describes a computer program; SF-185. FIPS Software Summary, is attached.

11. ABSTRACT (A 200-word or less factual summary of most significant information. If document includes a significant bibliography or literature survey. mention it here)

This publication outlines the organization and programs of the Polymer Science and Standards Division of the National Bureau of Standards. It contains summaries of all technical projects in the Division for the 1981 fiscal year.

12. KEY WORDS (Six to twelve entries; alphabetical order; capitalize only proper names; and separate key words by semicolons) polymers, plastics, macromolecules, standards

13. AVAILABILITY

—Unlimited

[X] For Official Distribution. Do Not Release to NTIS

$\square$ Order From Superintendent of Documents, U.S. Government Printing Office, Washington, D.C. 20402.

Order From National Technical Information Service (NTIS), Springfield, VA. 2216I
14. NO. OF

PRINTED PAGES

83

15. Price 

